Pacific

Journal of

Mathematics

\title{
GOOD TRACES FOR \\ NOT NECESSARILY SIMPLE DIMENSION GROUPS
}

DAVID HANDELMAN

Volume $281 \quad$ No. 2

April 2016 


\title{
GOOD TRACES FOR NOT NECESSARILY SIMPLE DIMENSION GROUPS
}

\author{
DAVID HANDELMAN
}

\begin{abstract}
Akin's notion of good measure, introduced to classify measures on Cantor sets, has been translated to dimension groups and traces by Bezuglyi and the author, but emphasizing the simple (minimal dynamical system) case. Here we permit nonsimplicity. Goodness of tensor products of large classes of non-good traces (measures) is established. We also determine the pure faithful good traces on the dimension groups associated to xerox-type actions on AF C*-algebras; the criteria turn out to involve algebraic geometry and number theory.

We also deal with a coproduct of dimension groups, wherein, despite expectations, goodness of direct sums is nontrivial. In addition, we verify a conjecture of Bezuglyi and Handelman (2014) concerning good subsets of Choquet simplices, in the finite-dimensional case.

Introduction and definitions

1. Characterization of goodness 368

2. Tensor products 373

3. Examples from xerox actions of tori on UHF algebras 379

4. Direct sums and goodness 389

5. Good sets of traces 398

Appendix A. Connections with zero-dimensional topological dynamics 408

Appendix B. Order unit good traces on $\mathbb{Z}^{k} \quad 412$

Appendix C. Good simplices 414

Acknowledgment 417

References $\quad 417$
\end{abstract}

\section{Introduction and definitions}

Akin [1999; 2005] (see also [Akin et al. 2008], among others) introduced and studied the notion of good measures in connection with the classification of (probability) measures on Cantor sets up to homeomorphism. With the development in [Putnam

Supported in part by a Discovery grant from NSERC.

MSC2010: primary 19K14; secondary 06F20, 46L80, 37A55, 37A35, 46A55, $28 \mathrm{E} 99$.

Keywords: good measure, trace, dimension group, affine representation, Newton polyhedron, approximately divisible, order ideal, simplicial group. 
1989; Herman et al. 1992; Giordano et al. 1995], among others, of classification and construction of minimal actions with respect to strong orbit and orbit equivalence via Vershik maps and ordered Grothendieck groups of $\mathrm{AF} \mathrm{C}^{*}$-algebras, this and related properties were translated into the language of (traces on) dimension groups (a class of partially ordered abelian groups) in [Bezuglyi and Handelman 2014], henceforth abbreviated [BeH 2014]. In particular, the characterizations therein of goodness of traces on simple dimension groups provided relatively easy constructions of good and non-good measures on minimal systems. For more details, see the discussion in the introduction to [BeH 2014].

Recent work (e.g., [Medynets 2006; Frick and Ormes 2013; Petersen 2012]) has extended Vershik action(s) to nonminimal systems, and correspondingly to nonsimple dimension groups. Here we give computable criteria for goodness in the general (approximately divisible) case, and then use the criteria to give a surprising result that tensor products of (some) non-good traces are good; this applies to the ugly traces of [BeH 2014]. We also completely determine the pure faithful traces on fixed point algebras under xerox actions of tori: the latter include Pascal's triangle and variations corresponding to spatially and temporally homogeneous random walks with finite support on the lattice $\mathbb{Z}^{d}$.

From [Handelman 1985, Theorem III.3], the pure faithful traces correspond to points $r=\left(r_{i}\right)$ in the strictly positive orthant of $\mathbb{R}^{d}$; those that are good are precisely the algebraic points that satisfy two number-theoretic conditions, which in the case that $d=1$, reduce to (i) no other algebraic conjugate of $r=r_{1}$ is positive and (ii) if the leading and terminal coefficients of the polynomial implementing the random walk are $a_{0}$ and $a_{k}$, then there exists $s$ such that $a_{0}^{s} / r$ and $a_{k}^{s} r$ are both algebraic integers.

We also deal with a strict form of direct sum of dimension groups, determining when the corresponding sum of traces is good; there are some surprises here, as the direct sum can be good without either one being good. We find for each $m$, a collection of simple dimension groups with traces, $\left(G_{i}, \tau_{i}\right)$, such that for any strict direct sum of $m$ or fewer distinct summands, $\bigoplus_{i \in S} G_{i}$, the sum of the traces is not good, but for any sum of more than $m$ direct summands, the sum is good.

We then consider good sets of traces. The first problem is the definition; it should be consistent with the current definition in the simple case and in the singleton case, and we discuss various possibilities; finally, we settle on one. We show that for the class of dimension groups considered above (arising from random walks on $\mathbb{Z}^{d}$ ), with any reasonable definition, the notion is surprisingly restrictive, and even order unit goodness turns out to be sensitive to the Newton polyhedra of the polynomials (unlike the case for single traces).

There are three appendices. The first discusses connections with dynamical systems, mostly for simple dimension groups. The second characterizes order unit good traces on simplicial groups, and the resulting characterization suggests that 
there are no effective criteria for goodness or order unit goodness when there are discrete traces, in contrast to the approximately divisible situation discussed in the rest of this article. Appendix $\mathrm{C}$ verifies, in the case of a finite-dimensional trace space, a conjecture made in [BeH 2014, Section 7] concerning the structure of good subsets relative to a simplex.

Definitions. A partially ordered abelian group $G$ with positive cone $G^{+}$is unperforated if whenever $n$ is a positive integer and $g \in G$, then $n g \in G^{+}$entails $g \in G^{+}$. An order unit for $G$ is an element $u \in G^{+}$such that for all $g \in G$, there exists a positive integer $K$ such that $-K u \leq g \leq K u$. A trace (formerly, state) is a nonzero positive group homomorphism $\tau: G \rightarrow \mathbb{R}$; if $\tau(u)=1$ and $u$ is an order unit, we say $\tau$ is normalized (with respect to $u$ ). The trace $\tau$ is faithful if $\operatorname{ker} \tau \cap G^{+}=\{0\}$ (this is much weaker than being one-to-one, and corresponds to faithfulness of the corresponding measure when there is a dynamical system nearby).

When $(G, u)$ is a partially ordered abelian group with order unit, we may form $S(G, u)$, the compact convex set of normalized traces, equipped with the weak (or point-open) topology. We denote by $\operatorname{Aff} S(G, u)$ the Banach space of continuous convex-linear (affine) real-valued functions on $S(G, u)$. There is a natural representation $G \rightarrow$ Aff $S(G, u)$, given by $g \mapsto \hat{g}$, where $\hat{g}(\tau)=\tau(g)$. We call this the affine representation of $(G, u)$. If $h$ in $\operatorname{Aff} S(G, u)$ is strictly positive (as a function on $S(G, u)$ ), we write $h \gg 0$. When $G$ is unperforated, we may use the notation $g \gg 0$ or $0 \ll g$ to indicate that $g$ is an order unit; this is consistent, as $\hat{g} \gg 0$ if and only if $g$ is an order unit.

If $Y \subset S(G, u)$, we define $Y^{\vdash}=\{h \in \operatorname{Aff} S(G, u)|h| Y \equiv 0\}$; when $Y=\{\tau\}$, a singleton, we abbreviate this to $\tau^{\vdash}$. In this case, $\tau^{\vdash}$ is a codimension-one subspace of Aff $S(G, u)$ and is an order ideal if and only if $\tau$ is pure. Following the convention in [BeH 2014], we signal purity with the replacement notation $\tau^{\perp}$.

If $(G, u)$ is an unperforated ordered abelian group, we say $G$ is approximately divisible if its range in $\operatorname{Aff} S(G, u)$ is norm-dense; for dimension groups with order unit, this is equivalent to $\tau(G)$ being dense in $\mathbb{R}$ for all pure traces $\tau$, or equivalently, for all order units $g \in G$, there exist order units $a, b$ of $G$ such that $g=2 a+3 b$ (and there are many other equivalent formulations) [Handelman 2014, Corollary 6.2].

When $I$ is a subgroup (typically an order ideal) of a partially ordered abelian group $G$, we say $I$ has its own order unit $w$ or $w$ is a relative order unit of I if $w \in I$ is an order unit of $I$ with respect to the relative ordering inherited from $G$. This is to emphasize the fact that $w$ is not an order unit for $G$, merely for $I$.

If $G$ is an unperforated ordered abelian group, we say $G$ is nearly divisible if for every order ideal $(I, w)$ which has its own order unit, $(I, w)$ is approximately divisible; from the discussion above, an equivalent form not referring to order ideals is that for all $g \in G^{+}$, there exist $a, b \in G^{+}$such that $g=2 a+3 b$ and $g \leq k a, k b$ for some positive integer $k$. This appears to be a new concept. 
For example, if $G=H \otimes U$, where $H$ is a partially ordered unperforated abelian group and $U$ is a noncyclic subgroup of the rationals $\mathbb{Q}$, then $G$ is nearly divisible, and it is approximately divisible if it has an order unit. We will see plenty of nearly divisible examples that are not of this type in later sections.

A trace on $G$ is discrete if its image $\tau(G)$ is a cyclic (that is, discrete) subgroup of $\mathbb{R}$. An alternative characterization of approximately divisible, for dimension groups, is that $(G, u)$ admit no discrete traces; for nearly divisible, the characterization is that no nonzero order ideal with order unit admits a discrete trace.

For general relevant results on partially ordered abelian groups, especially dimension groups, see [Goodearl 1986].

An interval in a partially ordered group $G$ is a subset of the form $[0, b]:=$ $\{g \in G \mid 0 \leq g \leq b\}$ for some $b \in G^{+}$.

Following [BeH 2014], and based on Akin's notion for measures on Cantor sets, a trace $\tau: G \rightarrow \mathbb{R}$ is $\operatorname{good}$ (as a trace of $G$ ) if for all $b \in G^{+}$, we have $\tau([0, b])=$ $[0, \tau(b)] \cap \tau(G)$; that is, if $a^{\prime} \in G$ and $0 \leq \tau\left(a^{\prime}\right) \leq \tau(b)$, there exists $a \in[0, b]$ such that $a-a^{\prime} \in \operatorname{ker} \tau$. If $(G, u)$ is a partially ordered abelian group with order unit, we say $\tau$ is order unit good if in the definition of good, we restrict $b$ to be an order unit.

\section{Characterization of goodness}

Order unit goodness is relatively easy to characterize when $(G, u)$ is an approximately divisible dimension group [BeH 2014, Proposition 1.7]: $\tau$ is order unit good if and only if the image of $\operatorname{ker} \tau$ in $\operatorname{Aff} S(G, u)$ is dense in

$$
\tau^{\vdash}:=\{h \in \operatorname{Aff} S(G, u) \mid h(\tau)=0\}
$$

(the latter is a closed codimension-one subspace of $\operatorname{Aff} S(G, u)$ ). This makes examples and non-examples relatively easy to construct. There is a corresponding characterization for goodness, which we shall simplify a bit, and use to actually do something.

Proposition 1.1. Suppose $(G, u)$ is a dimension group with order unit. Let $\tau$ be a faithful trace of $G$. Then $\tau$ is good if and only if for all nonzero order ideals with order unit $(I, w)$, both $\tau(I)=\tau(G)$ and $\tau \mid I$ is order unit good. If $\tau$ is pure, then sufficient for goodness is that there exist an order ideal I such that $\tau \mid I$ is good and $\tau\left(I^{+}\right)=\tau\left(G^{+}\right)$.

Remark. Necessity is shown in [BeH 2014, Proposition 4.2]; although the statement hypothesizes that $\tau$ be pure, this is not used in the proof (it is used there in the proof of sufficiency); also shown there was that if $\tau$ is good, then $\tau \mid I$ is good (as a trace on the order ideal $I$ ), and this implies (in the case that $I$ is approximately divisible) that $\tau \mid I$ is order unit good, just from the definitions. 
Remark. It is always possible to reduce to the case that $\tau$ is faithful by factoring out the maximal order ideal $J$ contained in $\operatorname{ker} \tau$ [BeH 2014, Lemma 4.4]. In this case, the criteria apply to $G / J$ (replacing $G$ ). This would make the statement somewhat more complicated.

Proof. Proof of necessity is given in [BeH 2014, Proposition 4.2], requiring neither purity of $\tau$ nor approximate divisibility.

Conversely, suppose $a \in G, b \in G^{+}$and $0<\tau(a)<\tau(b)$. Form the order ideal $I$ generated by $b$, that is, $I=\{c \in G \mid \exists N \in \mathbb{N}$ such that $-N b \leq g \leq N b\}$. Then $I$ is an order ideal with its own order unit, $b$. Since $\tau\left(I^{+}\right)=\tau\left(G^{+}\right)$, we have $\tau(I)=\tau(G)$, and thus there exists $a_{1} \in I$ such that $\tau\left(a_{1}\right)=\tau(a)$. Now order unit goodness of $\tau \mid I$ yields $a^{\prime} \in I$ such that $\tau\left(a^{\prime}\right)=\tau\left(a_{1}\right)=\tau(a)$ and $0 \leq a^{\prime} \leq b$, verifying goodness of $\tau$.

The final statement is just the sufficiency condition of [BeH 2014, Proposition 4.2].

Let $G$ be a dimension group, and let $I$ and $J$ be order ideals thereof. Then $H:=I+J$ (the set of sums of elements in $I$ and $J$ ) and $I \cap J$ are both order ideals. Most of the following are variations on [BeH 2014, Lemma 1.3]. As in [BeH 2014], an element $v$ of $G^{+}$is $\tau$-good or $\tau$-order unit good if $\tau([0, v])=[0, \tau(v)] \cap \tau(G)$.

Lemma 1.2. Suppose $G$ is a dimension group, and $I$ and $J$ each have relative order units, $w, y$ respectively. Then:

(a) $I+J$ is an order ideal of $G$ with a relative order unit.

(b) Let $\tau$ be a trace on $G$ such that $\operatorname{ker} \tau \cap G^{+}=\{0\}$ and $\tau(I) \cap \tau(J)$ is dense in $\mathbb{R}$. If $\tau \mid I$ and $\tau \mid J$ are good (as traces on $I$ and $J$ respectively), then $\tau \mid(I+J)$ is good.

(c) If $I+J$ is approximately divisible, then every order unit $b$ of $I+J$ can be written in the form $b=u+v$, where $u$, $v$ are relative order units for $I, J$ respectively.

(d) If $v$ is $\tau$-order unit good (with respect to I) and $w$ is $\tau$-order unit good (with respect to $J)$, and $\tau(I) \cap \tau(J)$ is dense in $\mathbb{R}$, then $v+w$ is $\tau$-order unit good with respect to $I+J$.

(e) Suppose that each of $I, J$ and $I+J$ is approximately divisible, and $\tau$ is a trace on $I+J$ such that each of $\tau \mid I$ and $\tau \mid J$ is order unit good, and $\tau(I) \cap \tau(J)$ is dense in $\mathbb{R}$. Then $\tau$ is order unit good as a trace of $I+J$.

Remark. Part (c) can fail if approximate divisibility is dropped; for example, take $G=\mathbb{Z}^{3}$ with the usual simplicial ordering, let $I$ be the order ideal generated by $(1,1,0)$ and let $J$ be the order ideal generated by $(0,1,1)$; then $I+J=G$ and the order unit $(1,1,1)$ cannot be realized as a sum of relative order units from $I$ and $J$ respectively. 
Proof. (a) That $I+J$ is an order ideal is ancient; see, e.g., [Goodearl 1986]. If $w$ and $y$ are respective order units for $I$ and $J$, then $z:=w+y$ is an order unit for $I+J$. To see this, let $f \in(I+J)^{+}$; for dimension groups, $(I+J)^{+}=I^{+}+J^{+}$, hence we can find $e \in I^{+}$and $g \in J^{+}$such that $f=e+g$. Since there exist positive integers $k, k^{\prime}$ such that $e \leq k w$ and $g \leq k^{\prime} v$, we have $f \leq k^{\prime \prime} z$, where $k^{\prime \prime}=\max \left\{k, k^{\prime}\right\}$.

(b) Select $b \in G^{+}$and $a \in G$ such that $\tau(a)<\tau(b)$. We may write $b=i+j$, where $i \in I^{+}$and $j \in J^{+}$. Then $\tau(i), \tau(j)>0$. We may write $\tau(a)=r+s$, where $r \in \tau(I)$ and $s \in \tau(J)$.

Assume $\tau(a) \geq \tau(i)$. By density of $\tau(I) \cap \tau(J)$, given

$$
0<\epsilon<\min \{\tau(i), \tau(b)-\tau(a)\},
$$

there exists $\delta \in \tau(I) \cap \tau(J)$ such that $\tau(i)-\epsilon<r+\delta<\tau(i)$. Then $s-\delta=\tau(a)-r-\delta$ satisfies

$$
\tau(a)-\tau(i)+\epsilon>s-\delta>\tau(a)-\tau(i)>0 .
$$

Hence we can write $\tau(a)=(r+\delta)+(s-\delta)$, where the parenthesized terms are respectively in the intervals $(0, \tau(i))$ and $(0, \tau(a)-\tau(i)+\epsilon)$. However, $\epsilon<\tau(b)-\tau(a)$ entails $\tau(a)-\tau(i)+\epsilon<\tau(b)-\tau(i)=\tau(j)$. Since $\pm \delta \in \tau(I \cap J)$, we may thus find $a_{1} \in I$ and $a_{2} \in J$ such that $0<\tau\left(a_{1}\right)<\tau(i)$ and $0<\tau\left(a_{2}\right)<\tau(j)$. Since each of $\tau \mid I$ and $\tau \mid J$ is good, there exist $c_{1} \in[0, i]$ (the interval in $I$ ) and $c_{2} \in[0, j]$ such that $\tau\left(c_{1}\right)<\tau(i)$ and $\tau\left(c_{2}\right)<\tau(j)$. Hence we have $c:=c_{1}+c_{2} \in[0, b]$ and $\tau(c)=\tau\left(c_{1}\right)+\tau\left(c_{2}\right)<\tau(i)+\tau(j)=\tau(b)$, verifying goodness in this case.

Reversing the roles of $i$ and $j$, the same conclusion results if $\tau(a) \geq \tau(j)$, so we are reduced to the case that $\tau(a)<\min \{\tau(i), \tau(j)\}$. If $\tau(a)=0$, there is nothing to do (except set $c=0$ ). Otherwise, choose $0<\epsilon<\tau(a) / 2$, find real $\delta \in \tau(I \cap J)$ such that $\tau(a) / 2-\epsilon<\delta+r<\tau(a) / 2$, and consider $\tau(a)=(r+\delta)+(s-\delta)$; then $r+\delta \in(0, \tau(a) / 2) \subset(0, \tau(i))$, so $s-\delta \in(\tau(a) / 2, \tau(a)) \subset(0, \tau(j))$. Now we can proceed as in the previous paragraph.

(c) Now let $b$ be an order unit of $I+J$. By approximate divisibility of $I+J$, the range of $I+J$ in Aff $S(I+J, b)$ is dense; hence given $\epsilon>0$, we may find $b_{0} \in I+J$ such that $(1 / 2-\epsilon) \mathbf{1}<\hat{b}_{0}<\mathbf{1} / \mathbf{2}$ (where ^ refers only to the representation on $S(I+J, b)$, that is, $\hat{b}=\mathbf{1})$. Let $\epsilon<1 / 8$, so that $\hat{b}_{0} \gg 0$ and thus $b_{0}$ is an order unit of $I+J$, and moreover, $2 b_{0} \leq b$, and $b-b_{0}$ is also an order unit for $I+J$.

Now consider the set $\mathcal{S}:=\left\{c \in I^{+} \mid c \leq b_{0}\right\}$. This is directed, as if $c, c^{\prime} \in \mathcal{S}$, then we have $c, c^{\prime} \leq b_{0}, c+c^{\prime}$; interpolating, we obtain $c^{\prime \prime}$ such that $c, c^{\prime} \leq c^{\prime \prime} \leq b_{0}, c+c^{\prime}$; as $c+c^{\prime} \in I$, it follows that $c^{\prime \prime} \in I$, so $c^{\prime \prime} \in \mathcal{S}$. As there exists $k$ such that $w \leq k b_{0}$, we can write $w=\sum_{i=1}^{k} w_{i}$, where $w_{i} \in I^{+}$and each $w_{i} \leq b_{0}$. Then $w_{i} \in \mathcal{S}$, so there exists $u_{0} \in I^{+}$such that $w_{i} \leq u_{0} \leq b_{0}$ for all $i$. Since $\sum w_{i}=w$ is an order unit for $I$, we know that $k u_{0}$ is an order unit for $I$, and thus $u_{0}$ is too. Hence there exists an order unit $u_{0}$ of $I$ such that $u_{0} \leq b_{0}$. 
Since $b-b_{0}$ is also an order unit for $I+J$, applying the same process to $J$ instead of $I$ yields an order unit $v_{0}$ of $J$ such that $v_{0} \leq b-b_{0}$. Thus $u_{0}+v_{0} \leq b_{0}+\left(b-b_{0}\right)=b$. The element $b-\left(u_{0}+v_{0}\right)$ is in the positive cone of $I+J$, so it can be written as $b-\left(u_{0}+v_{0}\right)=c+d$, where $c \in I^{+}$and $d \in J^{+}$. This yields $b=\left(u_{0}+c\right)+\left(v_{0}+d\right)$; setting $u=u_{0}+c$, we see that $u \in I^{+}$and is larger than an order unit for $I$, and so is itself an order unit for $I$; similarly $v=v_{0}+d$ is an order unit for $J$.

(d)-(e) Select an order unit $b$ for $I+J$, and $a \in I+J$ such that $0<\tau(a)<\tau(b)$. By (c), we may write $b=u+v$, where $u$ and $v$ are order units for $I$ and $J$ respectively. We can write $a=r+s$, where $r \in I$ and $s \in J$, and set $t=\tau(u)$ (as $\tau \mid I$ is order unit good, it does not vanish identically, hence $t>0$ ), so that $\tau(v)=\tau(b)-t$, which is again positive. Now proceed as in the proof of (b).

The density requirement on $\tau(I) \cap \tau(J)$ is essential.

Lemma 1.3. Suppose that $u$ and $v$ are elements of $G^{+}$, and let $\tau$ be a trace such that each is $\tau$-order unit good on the order ideals they generate, $I(u)$ and $I(v)$ respectively.

(a) If $u+v$ is $\tau$-order unit good on $I(u)+I(v)=I(u+v)$ and $\tau(I(u))+\tau(I(v))$ is dense in $\mathbb{R}$, then $\tau(I(u)) \cap \tau(I(v)) \neq\{0\}$.

(b) If, additionally, both $\tau(I(u))$ and $\tau(I(v))$ are dense subgroups of $\mathbb{R}$, then so is $\tau(I(u)) \cap \tau(I(v))$.

Proof. Suppose the intersection consists of just 0 . We may find positive real numbers $s \in \tau(I(u))$ and $t \in \tau(I(v))$ such that $s>\tau(u), t>\tau(v)$, and $0<r:=s-t<\tau(u+v)$ (since the value group is dense). By order unit goodness, there exists $a$ such that $0 \leq a \leq u+v$ and $\tau(a)=r$. Riesz decomposition entails $a=a_{1}+a_{2}$, where $0 \leq a_{1} \leq u$ and $0 \leq a_{2} \leq v$. Set $s^{\prime}=\tau\left(a_{1}\right) \geq 0$ and $t^{\prime}=\tau\left(a_{2}\right) \geq 0$. Then $s-t=s^{\prime}+t^{\prime}$, so $s-s^{\prime}=t+t^{\prime}$. The intersection consisting of 0 forces $s=s^{\prime}$ and $t=-t^{\prime}$; the latter forces $t=t^{\prime}=0$, a contradiction.

Now suppose the intersection is nonzero and not dense. Then it is cyclic, so there exists $x \in \mathbb{R}$, which we may assume to be positive, such that $\tau(I(u)) \cap \tau(I(v))=x \mathbb{Z}$. We may find $0<s, t<x$ with $s \in \tau(I(u))$ and $t \in \tau(I(v))$ such that $0<r:=s-t$. Find $a \leq u+v$ as above with $r=\tau(a)$, similarly decompose $a=a_{1}+a_{2}$, and define $s^{\prime}, t^{\prime}$ as in the preceding paragraph. We deduce $s-s^{\prime}=t+t^{\prime}$, hence there exists an integer $m$ such that $s-s^{\prime}=m x=t+t^{\prime}$; as $t, t^{\prime} \geq 0$, we have $m \geq 0$, but as $s<x$, we have $m<1$, hence $m=0$. This forces $t=t^{\prime}=0$, again a contradiction.

Corollary 1.4. Let $G$ be a nearly divisible dimension group with a faithful trace $\tau$. Suppose that I and $J$ are order ideals with their own order units such that each of $\tau|I, \tau| J$, and $\tau \mid(I+J)$ is order unit good. Then $\tau(I) \cap \tau(J)$ is a dense subgroup of $\mathbb{R}$. 
Proof. Since $\tau$ is faithful, $\tau \mid I$ and $\tau \mid J$ are nonzero, and since every trace on an order ideal with order unit is nondiscrete (as the order ideals are approximately divisible by definition), it follows that $\tau(I)$ and $\tau(J)$ are dense. Now Lemma 1.3(b) applies.

Let $(G, u)$ be a dimension group. Let $\mathcal{J}$ be a collection of nonzero order ideals, each with their own order unit, such that every order ideal of $G$ with order unit can be expressed as a sum of order ideals from $\mathcal{J}$ (such a sum can always be made finite, as the order ideal has an order unit); then we say $\mathcal{J}$ is a generating set of order ideals of $G$.

The criteria in Lemma 1.2 for goodness can be reduced to that on a generating set of order ideals. This will make the computations of Section 4 much simpler.

Lemma 1.5. Let $(G, u)$ be a nearly divisible dimension group, let $\mathcal{J}$ be a generating set of order ideals of $G$, and let $\tau$ be a faithful trace of $G$. For $\tau$ to be a good trace of $G$, it is sufficient that it satisfy

(i) for all $J \in \mathcal{J}$, we have $\tau(J)=\tau(G)$ and

(ii) for all $J \in \mathcal{J}$, we have $\tau \mid J$ is an order unit good trace of $J$.

Proof. We can express a nonzero order ideal $I$ with order unit as $I=\sum J_{\alpha}$ for some $J_{\alpha} \in \mathcal{J}$. Thus $\tau(I)=\sum \tau\left(J_{\alpha}\right)=\tau(G)$.

Since $I$ has an order unit, the sum can be made finite; now we apply induction (on the number of summands) to Lemma 1.2(d); this verifies the second property in Proposition 1.1.

Verifying the criteria for goodness and related properties is much simpler when the partially ordered abelian group is an ordered ring having 1 as an order unit.

Lemma 1.6. Let $(R, 1)$ be a (commutative) partially ordered commutative ring with 1 as order unit. If $R$ is approximately divisible, then it is nearly divisible.

Proof. Approximate divisibility implies the existence of order units $u$ and $v$ such that $1=2 u+3 v$; for any $r \in R^{+} \backslash\{0\}$, we thus have $r=2(r u)+3(r v)$. From $1 \leq k u, k v$ for some positive integer $k$, we deduce $r \leq k(r u), k(r v)$, verifying the definition of nearly divisible.

The following is implicit in the proof of [BeH 2014, Corollary 7.12].

Lemma 1.7. Let $(R, 1)$ be a partially ordered (commutative) unperforated ring with 1 as order unit, that is, an approximately divisible dimension group. Let $\tau$ be a faithful pure trace. Then $\tau$ is order unit good if and only if for all $\sigma \in \partial_{e} S(R, 1) \backslash\{\tau\}$, we have $\sigma(\operatorname{ker} \tau) \neq\{0\}$.

Proof. Since 1 is an order unit of the partially ordered ring, $X:=\partial_{e} S(R, 1)$ is compact and consists precisely of the normalized multiplicative traces of $R$; moreover, Aff $S(R, 1)=C(X, \mathbb{R})$ with the affine representation reinterpreted as $\tilde{g}(\phi)=\phi(g)$ for $\phi \in X$ (note the use of ${ }^{\sim}$ rather than ${ }^{\wedge}$, to distinguish them). By 
approximate divisibility, the image of $R$ is dense in $C(X, \mathbb{R})$. If $A$ is any ideal of $R$, then its closure in $C(X, \mathbb{R})$ is an ideal therein, and hence is of the form $\operatorname{Ann}(Y):=\{f \in C(X, \mathbb{R})|f| Y \equiv 0\}$ for a unique compact subset $Y$ of $X$.

Since $\tau$ is pure, it is multiplicative, and therefore $\operatorname{ker} \tau$ is an ideal of $R$ (not an order ideal, unless $\operatorname{ker} \tau=0$, as $\operatorname{ker} \tau \cap R^{+}=\{0\}$ is the definition of faithfulness). The closure of the image of $\operatorname{ker} \tau$ in $C(X, \mathbb{R})$ can thus be written in the form $\operatorname{Ann}(Y)$ for some compact subset $Y$.

If $\tau$ is order unit good, then $\operatorname{Ann}(Y)$ is $\operatorname{Ann}(\{\tau\})$ (corresponding to $\tau^{\perp}$ in Aff $S(R, 1))$, from which it follows that $Y=\{\tau\}$. Hence if $\sigma \in X \backslash\{\tau\}$, there exists a continuous $f: X \rightarrow[0,1]$ such that $f(\tau)=0$ but $f(\sigma)=1$; then $f \in \operatorname{Ann}(\{\tau\})$, hence there exist $g_{n} \in R$ such that $g_{n} \in \operatorname{ker} \tau$ and $\tilde{g}_{n} \rightarrow f$ uniformly. Applying $\sigma$, there exists $n$ such that $\sigma\left(g_{n}\right) \neq 0$, so that $\sigma(\operatorname{ker} \tau) \neq\{0\}$.

Conversely, suppose for every $\sigma \in X \backslash\{\tau\}$, we have $\sigma(\operatorname{ker} \tau) \neq\{0\}$. Then $\sigma \notin Y$, hence $Y=\{\tau\}$, so that the closure of the image of ker $\tau$ is codimension one in $C(X, \mathbb{R})$, hence equal to $\tau^{\perp}$ in $\operatorname{Aff} S(G, u)$. Thus $\tau$ is order unit good.

\section{Tensor products}

If $G$ and $H$ are partially ordered abelian groups, we may form the tensor product (as $\mathbb{Z}$-modules) $G \otimes_{\mathbb{Z}} H$ (usually, we delete the subscripted $\mathbb{Z}$ ); it is equipped with a cone which makes it into a partially ordered group, $\left\{\sum g_{i} \otimes h_{i} \mid g_{i} \in G^{+}\right.$and $\left.h_{i} \in H^{+}\right\}$ [Goodearl and Handelman 1986, Proposition 2.1]. If both are dimension groups, then so is $G \otimes H$, and if $u, v$ are respectively order units for $G, H$, then $u \otimes v$ is an order unit for $G \otimes H$. If $\sigma, \tau$ are respective (normalized) traces on $(G, u)$ and $(H, v)$, then $\sigma \otimes \tau$ (defined in the obvious way) is a (normalized) trace of $(G \otimes H, u \otimes v)$.

Appendix A informally discusses connections between tensor products of dimension groups and products of $\mathbb{Z}$-actions on Cantor minimal systems.

A special case occurs when we form the divisible hull of a dimension group, $G \otimes \mathbb{Q}$, the rational vector space that $G$ generates. Then $\tau$ extends to a trace $G \otimes \mathbb{Q}$ in the obvious way, denoted $\tau \otimes 1_{\mathbb{Q}}$. In general, $\tau$ being order unit good or good implies the corresponding property for $\tau \otimes 1_{\mathbb{Q}}$, but the converse fails practically generically. As a special case, we [BeH 2014] defined a trace $\tau$ to be $u g l y$ if $\tau \otimes 1_{\mathbb{Q}}$ is good and $\operatorname{ker} \tau$ has discrete image in (the Banach space) Aff $S(G, u)$. Ugly traces exist in profusion.

In Akin's original context of measures on Cantor sets, he showed that (what amounts to) the tensor product of good traces is good; in the context of simple dimension groups or more generally for approximately divisible dimension groups, the tensor product of order unit good traces was shown to be order unit good $[\mathrm{BeH}$ 2014, Proposition 5.2]. Here, we show a somewhat surprising result for order unit goodness: if $(G, u)$ and $(H, v)$ are approximately divisible, and both $\sigma \otimes 1_{\mathbb{Q}}$ and 
$\tau \otimes 1_{\mathbb{Q}}$ are order unit good on their respective groups, then $\sigma \otimes \tau$ is order unit good (as a trace on $G \otimes H$ ). This means that the tensor product has a stronger property (in general) than its constituents. In particular, the tensor product of ugly traces is at least order unit good.

A weaker notion is refinability; again based on Akin's definition in the dynamical situation, and translated to partially ordered groups: a trace $\tau$ on $(G, u)$ is refinable if whenever $b \in G^{+} \backslash \operatorname{ker} \tau$ and $\left\{a_{i}\right\}$ is a finite subset of $G^{+}$such that $\tau(b)=\sum \tau\left(a_{i}\right)$, there exist $\left\{a_{i}^{\prime}\right\} \subset G^{+}$such that $b=\sum a_{i}^{\prime}$ and $\tau\left(a_{i}\right)=\tau\left(a_{i}^{\prime}\right)$. Surprisingly, the corresponding tensor product results actually fail for refinability (even though the set of refinable traces is a dense $\mathrm{G}_{\delta}$ in the trace space).

Using the criterion of Proposition 1.1, we then obtain a corresponding criterion for goodness of the tensor product $\left(G\right.$ and $H$ are nearly divisible, $\sigma \otimes 1_{\mathbb{Q}}$ and $\tau \otimes 1_{\mathbb{Q}}$ are good, and a condition that guarantees the value groups on the order ideals is the same as the full value group).

Proposition 2.1. Let $(G, u)$ and $(H, v)$ be approximately divisible dimension groups with traces $\sigma$ and $\tau$ respectively. If each of $\sigma \otimes 1_{\mathbb{Q}}$ and $\tau \otimes 1_{\mathbb{Q}}$ on $G \otimes \mathbb{Q}$ and $H \otimes \mathbb{Q}$ respectively is order unit good, then the trace on $(G \otimes H, u \otimes v)$ given by $\sigma \otimes \tau$ is order unit good.

If we only require that $\sigma \otimes \tau \otimes 1_{\mathbb{Q}}$ (a trace on $G \otimes H \otimes \mathbb{Q}$ ) be order unit good (in place of each of $\sigma \otimes 1_{\mathbb{Q}}$ and $\tau \otimes 1_{\mathbb{Q}}$ being good), the conclusion is false; an example will be given later (Example 2.6).

We require a number of elementary results about tensor products. Here the tensors will be over one of the rings $\mathbb{Z}, \mathbb{Q}$, or $\mathbb{R}$; torsion-free (module) means torsion-free abelian group when the underlying ring is $\mathbb{Z}$; otherwise, it just means vector space over the relevant field.

Lemma 2.2. Let $A$ and $B$ be torsion-free modules, and $A^{\prime} \subset A, B^{\prime} \subset B$ submodules such that $A / A^{\prime}$ and $B / B^{\prime}$ are torsion-free.

(a) The kernel of the map $A \otimes B \rightarrow A \otimes\left(B / B^{\prime}\right)$ is $A \otimes B^{\prime}$.

(b) The kernel of the map $A \otimes B \rightarrow\left(A / A^{\prime}\right) \otimes\left(B / B^{\prime}\right)$ is $A \otimes B^{\prime}+A^{\prime} \otimes B$.

Proof. (a) One inclusion is obvious. Because the quotient is torsion-free, $A \otimes B / B^{\prime}$ is torsion-free. We have an induced map $(A \otimes B) /\left(A \otimes B^{\prime}\right) \rightarrow A \otimes\left(B / B^{\prime}\right)$. If $z$ is in the kernel, find a nonzero integer $n$ such that $n z$ has a representative in $A \otimes B$ of least length (as $n$ varies over nonzero integers), say $n z=\sum a_{i} \otimes b_{i}+\left(A \otimes B^{\prime}\right)$. Then $\left\{a_{i}\right\}$ is rationally linearly independent; hence the image, $n \bar{z}$, yields $0=\sum a_{i} \otimes\left(b_{i}+B^{\prime}\right)$. Since $B / B^{\prime}$ is torsion-free, this easily implies all $b_{i}+B^{\prime}=0$ (tensor with $\mathbb{Q}$ if necessary, so we are working over a field, then use a basis for $B^{\prime} \mathbb{Q}$, extended to $B \mathbb{Q}$ ). (This proof works for all fields.) 
(b) First, $A \otimes B /\left(A \otimes B^{\prime}\right)$ is naturally isomorphic to $A \otimes B / B^{\prime}$ by (a). Then another application of (a) with the order reversed yields a natural isomorphism $\left(A \otimes\left(B / B^{\prime}\right)\right) /\left(A^{\prime} \otimes\left(B / B^{\prime}\right)\right) \cong\left(A / A^{\prime}\right) \otimes\left(B / B^{\prime}\right)$. Then the kernel of the first map is $A \otimes B^{\prime}$, and that of the second is $A^{\prime} \otimes\left(B / B^{\prime}\right)$, which pulls back to $A \otimes B^{\prime}+A^{\prime} \otimes B$.

Proof of Proposition 2.1. We will show that the closure of the image of $\operatorname{ker} \sigma \otimes \tau$ in Aff $S(G \otimes H, u \otimes v)$ is $(\sigma \otimes \tau)^{\vdash}$; by [BeH 2014, Proposition 1.7], $\sigma \otimes \tau$ is order unit good.

First, we identify the product Aff $S(G, u) \otimes_{\mathbb{R}} \operatorname{Aff} S(H, v)$ with a subspace of Aff $S(G \otimes H, u \otimes v)$ in the obvious way. Standard results (e.g., pure traces are pure tensors) yield that it is a dense subspace.

We note that $(\operatorname{ker} \sigma) \otimes H+G \otimes(\operatorname{ker} \tau) \subseteq \operatorname{ker} \sigma \otimes \tau$. It easily follows that the closure of the image of $(\operatorname{ker} \sigma) \otimes H$ contains everything in $y \otimes \operatorname{Aff} S(H, v)$ (real tensors), where $y$ varies over the image of $\operatorname{ker} \sigma$ (in $\sigma^{\vdash} \subset \operatorname{Aff} S(G, u)$ ). For $y$ fixed, $y \otimes \operatorname{Aff} S(H, v)$ is a real vector space, and this means that we can rewrite it as $y \mathbb{R} \otimes \operatorname{Aff} S(H, v)$ (just approximate real multiples of $\hat{v}$ by elements of $\widehat{H}$, and transfer through the tensor product). Taking finite sums, we see that the closure of the image of $\operatorname{ker} \sigma \otimes H$ includes the closure of $\operatorname{Im}(\operatorname{ker} \sigma) \mathbb{Q} \otimes \operatorname{Aff} S(H, v)$.

Now $\sigma \otimes 1_{\mathbb{Q}}$ being order unit good implies $(\operatorname{ker} \sigma) \otimes \mathbb{Q}$ has dense image in $\sigma^{\vdash}$ (in Aff $S(G, u)$ ). If $e$ is an element of $G \otimes \mathbb{Q}$, there exists a nonzero integer $m$ such that $m e \in G$. If in addition, $\sigma \otimes 1_{\mathbb{Q}}(e)=0$, then $\sigma(m e)=0$; thus $\operatorname{ker}\left(\sigma \otimes 1_{\mathbb{Q}}\right) \subseteq(\operatorname{ker} \sigma) \mathbb{Q}$ (the reverse inclusion is trivial, but never needed).

Thus the closure of the image of $(\operatorname{ker} \sigma) \otimes H$ contains $\operatorname{Im}(\operatorname{ker} \sigma) \mathbb{Q} \otimes \operatorname{Aff} S(H, v)$, which in turn contains the closure of $\operatorname{Im}(\operatorname{ker} \sigma) \mathbb{Q} \otimes \operatorname{Aff} S(H, v)$, and thus includes $\sigma^{\vdash} \otimes \operatorname{Aff} S(H, v)$.

Similarly, the closure of the image of $G \otimes \operatorname{ker} \tau$ contains Aff $S(G, u) \otimes \tau^{\vdash}$. Set $A=\operatorname{Aff} S(G, u), A^{\prime}=\sigma^{\vdash}, B=\operatorname{Aff} S(H, v)$, and $B^{\prime}=\tau^{\vdash}$; then each is a Banach space, and $\left(A / A^{\prime}\right)$ and $\left(B / B^{\prime}\right)$ are both one-dimensional, and the closure of the image of $\operatorname{ker}(\sigma \otimes \tau)$ contains $A^{\prime} \otimes B+A \otimes B^{\prime}$.

By (b) above, $(A \otimes B) /\left(A^{\prime} \otimes B+A \otimes B^{\prime}\right)$ is one-dimensional. Let $W=$ $A^{\prime} \otimes B+A \otimes B^{\prime}$ and $Z=\operatorname{Aff} S(G, u) \otimes \operatorname{Aff} S(H, v)$, so that $W$ is a codimensionone subspace of $Z$. It is now an easy exercise to show that when we complete $Z$ to Aff $S(G \otimes H, u \otimes v)$, the closure, $\bar{W}$, is of at most codimension one. (This is a general Banach space result; if $\bar{W} \neq \bar{z}$, then $W=\bar{W} \cap Z$ as $W$ is codimension one in $Z$; choose $z \in Z \backslash W$; the functional sending $z \mapsto 1$ and $W \mapsto 0$ is continuous (essentially the closed graph theorem), and hence extends to a bounded linear functional $p$ on $\bar{W}$; we may write arbitrary $y \in \bar{Z}$ as $\lim y_{n}$; then $y_{n}=p\left(y_{n}\right) z+\left(y_{n}-p\left(y_{n}\right) z\right)$, and thus by continuity, $y=p(y) z+(y-p(y) z)$, and $y-p(y) z$ is in $\bar{W}$, hence $z+\bar{W}=\bar{z}$.)

In particular, the closure of the image of $\operatorname{ker} \sigma \otimes \tau$ in Aff $S(G \otimes H, u \otimes v)$ is codimension one. As it is contained in $(\sigma \otimes \tau)^{\vdash}$, which is proper, it follows that the image of $\operatorname{ker} \sigma \otimes \tau$ is dense in $(\sigma \otimes \tau)^{\vdash}$. 
This explains a phenomenon exemplified in [BeH 2014, Example 9]. Let $G$ be a critical dimension group of rank $k+1$ (that is, a free rank $k+1$ abelian group densely embedded in $\mathbb{R}^{k}$, and equipped with the strict ordering therefrom [Handelman 1982]). Then we say $G$ is basic (as a critical group) if it is order-isomorphic to the subgroup of $\mathbb{R}^{k}$ spanned by $\left\{e_{i} ; \sum \alpha_{j} e_{j}\right\}$, where $\left\{e_{i}\right\}$ is the standard basis and $\left\{1, \alpha_{1}, \ldots, \alpha_{k}\right\}$ is linearly independent over the rationals (this guarantees density of the subgroup). Every critical group is topologically isomorphic to a group of the latter form.

For basic critical groups, every pure trace is ugly, as is immediate from the definitions. Hence if $G_{i}$ are basic critical groups (and there is more than one), then all of the pure traces of their tensor product (a simple dimension group) $\otimes G_{i}$ are good. In [BeH 2014, Example 9], an example was given of a basic critical group of rank three, for which all pure traces on $G \otimes G$ are good. We also asked whether the pure traces on $G \otimes G \otimes G$ are good, and now we know that the answer is yes.

It is plausible that among critical groups, basic ones are characterized by all pure traces being ugly; this is false, but is close to being true [Handelman 2013a, Proposition 7.4]. There are lots of critical groups for which all or some are bad, hence not ugly [BeH 2014, Section 2]. It can also happen that if both $\sigma, \tau$ are bad traces (a trace $\tau$ is bad if $\operatorname{ker} \tau$ consists of the infinitesimal elements of the group [BeH 2014]), then $\sigma \otimes \tau$ is good; but it can also arise that $\sigma \otimes \tau$ is not even ugly.

Now suppose that $(G, u)$ and $(H, v)$ are nearly divisible, and $\sigma, \tau$ are normalized traces on $G, H$ respectively such that $\sigma \otimes 1_{\mathbb{Q}}$ and $\tau \otimes 1_{\mathbb{Q}}$ are both good. We expect to obtain that $\sigma \otimes \tau$ is a good trace on $G \otimes H$.

Lemma 2.3. Let $(G, u)$ and $(H, v)$ be dimension groups with order unit. Then:

(a) $G \otimes H$ is approximately divisible if and only if at least one of $G$ or $H$ is.

(b) $G \otimes H$ is nearly divisible if and only if at least one of $G$ or $H$ is.

Proof. (a) Suppose $G$ is approximately divisible. Every pure trace of $(G \otimes H, u \otimes v)$ is of the form $\sigma \otimes \tau$ [Goodearl and Handelman 1986, Lemma 4.1], where $\sigma, \tau$ are pure traces of $G, H$ respectively. Then $(\sigma \otimes \tau)(G \otimes H)$ is $\sigma(G) \cdot \tau(H)$ (the set of sums of terms of the form $\sigma(g) \cdot \tau(h))$; as $\sigma(G)$ is dense, obviously so is $\sigma(G) \cdot \tau(H)$, so that $G \otimes H$ has no discrete pure traces, and is thus approximately divisible. The same argument applies if instead $H$ is approximately divisible.

If neither $G$ nor $H$ is approximately divisible, then there exists a discrete trace $\sigma$ of $G$ and a discrete trace $\tau$ of $H$; as these are normalized (at $u, v$ respectively), $\sigma(G)=(1 / n) \mathbb{Z}$ and $\tau(H)=(1 / m) \mathbb{Z}$ for some positive integers $m$ and $n$; then $(\sigma \otimes \tau)(G \otimes H)=(1 / m n) \mathbb{Z}$, which is discrete. Hence $G \otimes H$ admits a discrete trace, and thus is not approximately divisible.

(b) Select $a=\sum g_{i} \otimes h_{i} \in(G \otimes H)^{+}$; from the definition of the ordering on the tensor product, we can assume each of $g_{i}$ and $h_{i}$ are positive in their respective 
groups. By definition, we can write $g_{i}=2 a_{i}+3 b_{i}$, where $0 \leq g_{i} \leq k a_{i}, k b_{i}$ for some positive integer $k$; since the sum is finite, we can take the same integer $k$ for all $i$. Set $c_{1}=\sum a_{i} \otimes h_{i}$ and $c_{2}=\sum b_{i} \otimes h_{i}$. Then $a=2 c_{1}+3 c_{2}$; moreover, $\sum g_{i} \otimes h_{i} \leq k \sum a_{i} \otimes h_{i}$, that is, $a \leq k c_{1}$, and similarly $a \leq k c_{2}$.

If neither $G$ nor $H$ is nearly divisible, there exist an order ideal of $G$ with its own order unit, $(I, w)$ together with a discrete trace (of $I) \phi$, and an order ideal of $H$ with its own order unit, $(J, y)$ and a discrete trace on it, $\psi$. Then $\phi \otimes \psi$ is a discrete trace (as above) of $I \otimes J$; this being an order ideal of $G \otimes H$, the latter is not nearly divisible.

Lemma 2.4. Let $G$ and $H$ be nearly divisible, having faithful traces $\sigma$ and $\tau$ respectively such that $\sigma \otimes 1_{\mathbb{Q}}$ and $\tau \otimes 1_{\mathbb{Q}}$ are good as traces on $G \otimes \mathbb{Q}, H \otimes \mathbb{Q}$ respectively.

(a) Let $(I, w)$ be an order ideal of $G$ with its own order unit, and let $(J, y)$ be an order ideal of $H$ with its own order unit. Then $(\sigma \otimes \tau) \mid(I \otimes J)$ is order unit good.

(b) Suppose for each order ideal I of $G, \sigma(I)=\sigma(G)$, and similarly, for each order ideal $J$ of $H$, we have $\tau(J)=\tau(H)$. Then for every nonzero order ideal $L$ of $G \otimes H$, we have $(\sigma \otimes \tau)(L)=(\sigma \otimes \tau)(G \otimes H)$.

(c) Suppose the hypotheses of (b) apply. Let $(L, e)$ be an arbitrary order ideal of $G \otimes H$ with its own order unit. Then $(\sigma \otimes \tau) \mid L$ is order unit good.

Proof. (a) Each of the restrictions of $\sigma \otimes 1_{\mathbb{Q}}$ and $\tau \otimes 1_{\mathbb{Q}}$ to $I \otimes \mathbb{Q}$ and $J \otimes \mathbb{Q}$ respectively is good, hence order unit good, and thus $(\sigma \otimes \tau) \mid(I \otimes J)$ is an order unit good trace of $I \otimes J$.

(b) First, if $L=I \otimes J$ (where $I$ and $J$ are nonzero order ideals in $G$ and $H$ respectively), then $(\sigma \otimes \tau)(I \otimes J)$ is the subgroup of $\mathbb{R}$ generated by all terms of the form $\sigma(a) \cdot \tau(b)$, where $a \in I$ and $b \in J$, and $(\sigma \otimes \tau)(G \otimes H)$ has the same form, except $a$ and $b$ are allowed to vary over $G$ and $H$ respectively. Since for all $a \in G$, there exists $a^{\prime} \in I$ such that $\sigma\left(a^{\prime}\right)=\sigma(a)$, and similarly for $\tau$, the two groups are equal.

If $e \in L^{+}$, then by the definition of the tensor product ordering, we can write $e=\sum g_{i} \otimes h_{i}$. For an element $x$ in the positive cone of a dimension group, let $I(x)$ be the order ideal it generates; then it is easy to check (since sums of order ideals are again order ideals in a dimension group) that $L=I(e)=\sum I\left(g_{i}\right) \otimes I\left(h_{i}\right)$; in particular, $L$ contains a tensor product of order ideals, so the previous paragraph applies. (c) Every $e \in(G \otimes H)^{+}$can be written in the form $e=\sum g_{i} \otimes h_{i}$ with $g_{i} \in G^{+}$ and $h_{i} \in H^{+}$. By (a), the restriction of $\sigma \otimes \tau$ to each of $I\left(g_{i}\right) \otimes I\left(h_{i}\right)$ is order unit good. Since $\sigma \otimes \tau(L)=(\sigma \otimes \tau)(G \otimes H)$, for any nonzero order ideal $L$ of $G \otimes H$, we may apply Lemma 1.2(e) (the intersection of the value groups is dense), so the restriction of $\sigma \otimes \tau$ to $L$ is order unit good. 
Proposition 2.5. Suppose that $(G, u, \sigma)$ and $(H, v, \tau)$ are nearly divisible dimension groups with faithful trace having the following properties:

(i) For all nonzero order ideals $I$ and $J$ of $G$ and $H$ respectively, $\sigma(I)=\sigma(G)$ and $\tau(J)=\tau(H)$.

(ii) Each of $\sigma \otimes 1_{\mathbb{Q}}$ and $\tau \otimes 1_{\mathbb{Q}}$ is good on $G \otimes \mathbb{Q}, H \otimes \mathbb{Q}$ respectively.

Then $\sigma \otimes \tau$ is a good trace of $G \otimes H$.

Proof. This follows from Lemma 2.3, Lemma 2.4, and Proposition 1.1.

Even in the simple case, it is not true that goodness of $\tau \otimes \tau \otimes \mathbf{1}_{\mathbb{Q}}$ (a trace on $G \otimes G \otimes \mathbb{Q}$ ) implies $\tau \otimes \tau$ is good. In fact, the next example illustrates something more drastic.

A weaker property than goodness is refinability: a trace $\tau: G \rightarrow \mathbb{R}$ is refinable if whenever $b \in G^{+}$and there exist $\left\{a_{i}\right\} \subset G^{+}$such that $\tau(b)=\sum \tau\left(a_{i}\right)$, then there exist $a_{i}^{\prime} \in G^{+}$such that $\tau\left(a_{i}\right)=\tau\left(a_{i}^{\prime}\right)$ and $b=\sum a_{i}^{\prime}$. Good traces are refinable [BeH 2014, Lemma 7.3]. Following [BeH 2014], a trace $\tau$ is bad $\operatorname{if} \operatorname{Inf} G=\operatorname{ker} \tau$ and $\tau$ is not the only normalized trace. It is trivial that bad traces are refinable when $\operatorname{Inf} G=\{0\}$ [BeH 2014].

More interestingly, when there is more than one trace, bad traces are generic; in fact, they constitute a dense $\mathrm{G}_{\delta}$ of $S(G, u)$, merely under the assumption that $G$ is countable [Giordano et al. $\geq 2016$ ]. Because of this, one would expect refinability to be even better behaved under tensor products than goodness. This is not the case.

Example 2.6. There exists a simple dimension group $G$ with a pure trace $\tau$ with the following properties:

(a) $\tau$ is bad, and thus is refinable.

(b) $\tau \otimes \tau \otimes 1_{\mathbb{Q}}$, a trace on $G \otimes G \otimes \mathbb{Q}$, is good.

(c) The trace $\tau \otimes \tau: G \otimes G \rightarrow \mathbb{R}$ is not even refinable.

(d) The trace $\tau \otimes \tau \otimes \tau \otimes \tau$ on $G^{\otimes 4}$ is good.

Proof. Let $\alpha$ be real, quartic, and integral (that is, it satisfies a monic degree-four irreducible polynomial with integer coefficients), and let $\beta$ be a real number not satisfying any degree-four polynomial over the rationals (in particular, $\beta \notin \mathbb{Q}(\alpha)$, where the latter is the field generated over the rationals by $\alpha$ ). Let $G$ be the subgroup of $\mathbb{R}^{2}$ generated by $\left\{(1,1),(\alpha, \beta),\left(\alpha^{2}, \beta^{2}\right)\right\}$. The three $2 \times 2$ determinants are $\left\{\beta-\alpha, \beta^{2}-\alpha^{2}, \alpha \beta^{2}-\alpha^{2} \beta\right\}$; since $\beta \neq \alpha$, this set is rationally linearly independent (rational linear independence of $\{1, \alpha+\beta, \alpha \beta\}$ follows from $\beta \notin \mathbb{Q}(\alpha)$ ). Thus $G$ is dense in $\mathbb{R}^{2}$, so with the strict ordering it inherits from the latter, it will be a simple dimension group.

Let $\tau: G \rightarrow \mathbb{R}$ be the projection onto the first coordinate. This is a pure trace, and moreover, $\operatorname{ker} \tau=\{0\}$, so that $\tau$ is bad, and thus is refinable. 
Now make the identifications

$$
\begin{array}{ll}
(1,0) \otimes(1,0) \mapsto(1,0,0,0), & (1,0) \otimes(0,1) \mapsto(0,1,0,0), \\
(0,1) \otimes(1,0) \mapsto(0,0,1,0), & (0,1) \otimes(0,1) \mapsto(0,0,0,1) .
\end{array}
$$

This yields order isomorphisms $\mathbb{Z}^{2} \otimes \mathbb{Z}^{2} \rightarrow \mathbb{Z}^{4}$ and $\mathbb{R}^{2} \otimes \mathbb{R}^{2} \rightarrow \mathbb{R}^{4}$. Then $G \otimes G$, being a simple dimension group, inherits the strict ordering on $\mathbb{R}^{4}$, and is spanned by the nine elements

$$
\begin{array}{lll}
a=(1,1,1,1), & b=(\alpha, \beta, \alpha, \beta), & c=\left(\alpha^{2}, \beta^{2}, \alpha^{2}, \beta^{2}\right), \\
d=(\alpha, \alpha, \beta, \beta), & e=\left(\alpha^{2}, \alpha \beta, \alpha \beta, \beta^{2}\right), & f=\left(\alpha^{3}, \alpha \beta^{2}, \alpha^{2} \beta, \beta^{2}\right), \\
g=\left(\alpha^{2}, \alpha^{2}, \beta^{2}, \beta^{2}\right), & h=\left(\alpha^{3}, \alpha^{2} \beta, \alpha \beta^{2}, \beta^{3}\right), & j=\left(\alpha^{4}, \alpha^{2} \beta^{2}, \alpha^{2} \beta^{2}, \beta^{4}\right) .
\end{array}
$$

The trace $\tau \otimes \tau$ identifies with the projection on the first coordinate, which we will call $\sigma$. Since $\alpha$ satisfies an irreducible polynomial of degree four (and therefore of no less degree), say $\alpha^{4}=A \alpha^{3}+B \alpha^{2}+C \alpha+D$, with $A, B, C, D \in \mathbb{Z}$, it follows that

$$
\operatorname{ker} \sigma=\langle d-b, e-c, g-e, h-f, j-A f-B c-C b-D a\rangle \text {. }
$$

(Since $\sigma(G \otimes G) \subset \mathbb{Z}[\alpha]$ and the latter is rank four, the kernel has rank five; this reduces the problem to showing the cokernel of the group on the right is torsion-free, which is routine.) The first four of the generators are of the form $(0, *, *, 0)$, and it is easy to verify that the group that they span is dense in $0 \oplus \mathbb{R}^{2} \oplus 0$. The last generator has nonzero fourth coordinate (since $\beta$ satisfies no fourth degree polynomial); call it $\gamma$. Thus the closure of $\operatorname{ker} \sigma$ is $\{0\} \oplus \mathbb{R}^{2} \oplus \gamma \mathbb{Z}$.

In particular, the closure of $\operatorname{ker} \sigma$ is not a real vector space, so $\sigma=\tau \otimes \tau$ is not good; moreover, it is not even refinable, since $\phi(\operatorname{ker} \sigma)$ is cyclic and nonzero, where $\phi$ is the projection onto the fourth coordinate [BeH 2014, Proposition B.5].

On the other hand, $(\operatorname{ker} \sigma) \otimes \mathbb{Q}$ is dense in $\{0\} \oplus \mathbb{R}^{3}$, and since $\operatorname{ker}\left(\sigma \otimes 1_{\mathbb{Q}}\right)$ contains $(\operatorname{ker} \sigma) \otimes \mathbb{Q}$, it follows that $\sigma \otimes 1_{\mathbb{Q}}=\tau \otimes \tau \otimes 1_{\mathbb{Q}}$ is a good trace on $G \otimes \mathbb{Q}$. By Proposition 2.1, $\tau \otimes \tau \otimes \tau \otimes \tau$ is a good trace on $G \otimes G \otimes G \otimes G$.

Left open are the properties of $\tau \otimes \tau \otimes \tau$. Short of computing with a $\mathbb{Z}$-basis consisting of 27 elements, there did not seem to be any method of attack (the kernel has rank 23; however, the infinitesimal subgroup is substantial, too).

\section{Examples from xerox actions of tori on UHF algebras}

We characterize the good faithful pure traces on the dimension groups arising from xerox-product-type actions of tori on UHF C*-algebras. It turns out that there is a surprising number-theoretic component.

Appendix A points out strong analogies between Bernoulli measures and the traces discussed here. 
Form the Laurent polynomial ring in $d$ variables over the integers, $\mathbb{Z}\left[x_{i}^{ \pm 1}\right]$, and let $\mathbb{Z}\left[x_{i}^{ \pm 1}\right]^{+}$denote the set of those with only nonnegative coefficients. As in [Handelman 1985; 1987], we adopt monomial notation; that is, for $w \in \mathbb{Z}^{d}$, define $x^{w}=x_{1}^{w(1)} \cdot x_{2}^{w(2)} \cdots \cdots x_{d}^{w(d)}$. For any $f \in \mathbb{Z}\left[x_{i}^{ \pm 1}\right]$, we denote the coefficient of $x^{w}$ in $f$ by $\left(f, x^{w}\right)$ (inner product notation, which is consistent with the origins of the work), and we set $\log f:=\left\{w \in \mathbb{Z}^{d} \mid\left(f, x^{w}\right) \neq 0\right\}$. Let $P=\sum a_{w} x^{w} \in \mathbb{Z}\left[x_{i}^{ \pm 1}\right]^{+}$ (where $a_{w} \in \mathbb{Z}^{+}$), and form the ring $R_{P}=\mathbb{Z}\left[\left\{x^{w} / P\right\}_{w \in \log P}\right]$. Equipped with the partial ordering generated additively and multiplicatively by $\left\{x^{w} / P \mid w \in \log P\right\}$, this is a dimension group and an ordered ring with 1 as order unit, and many more properties (marked with bullets below). We may also form $\mathbb{Z}\left[x_{i}^{ \pm 1}, 1 / P\right]$ (a subring of the field of fractions of the Laurent polynomial ring). It also has a partial ordering given by $\left\{f / P^{k} \mid \exists N\right.$ such that $P^{N} f$ has no negative coefficients $\}$. The restriction of this to $R_{P}$ yields the original ordering.

This arose from the following construction. Let $n=P(1,1,1, \ldots, 1)$, and form $\mathcal{A}=\otimes M_{n} \mathbb{C}$ (the UHF $C^{*}$-algebra). The Laurent polynomial $P$ is the character of an $n$-dimensional representation of the torus $\mathbb{T}^{d}$, say given by $z \mapsto \operatorname{diag}\left(z^{w}\right)$ (one for each $w$ that appears in $P$, with repetitions as indicated by the multiplicities, that is, the coefficients). This yields a map $\pi: \mathbb{T}^{d} \rightarrow M_{n} \mathbb{C}$ with nonzero entries along the diagonal. Form $\phi:=\otimes \operatorname{Ad} \pi: \mathbb{T}^{d} \rightarrow$ Aut $\mathcal{A}$, and the corresponding fixed point subrings, $\mathcal{A}^{\phi\left(\mathbb{T}^{d}\right)}$, and $\mathcal{A} \times{ }_{\phi} \mathbb{T}^{d}$, the latter the $\mathrm{C}^{*}$-crossed product. Then $\left(\mathrm{K}_{0}\left(\mathcal{A}^{\phi\left(\mathbb{T}^{d}\right)}\right),[1]\right)$ is a naturally ordered ring isomorphic to $R_{P}$ and $\mathrm{K}_{0}\left(\mathcal{A} \times{ }_{\phi} \mathbb{T}^{d}\right)$ is similarly isomorphic to the ordered ring $\mathbb{Z}\left[x_{i}^{ \pm 1}, 1 / P\right]$. This will play a role in what follows.

Renault [1980] determined the positive cone and analyzed (inter alia) the structure of $R_{P}$ when $P=1+x$. The pure (ergodic) traces thereon were determined by Orey (in terms of the simple random walk) in the mid-1960s.

We normally assume that $P$ is projectively faithful; that is, $\log P-\log P$ generates (as an abelian group) the standard copy of $\mathbb{Z}^{d}$ in $\mathbb{R}^{d}$ (we can reduce to this case anyway). This has the effect that whenever $v \in \log P^{k} \cap$ int cvx $\log P^{k}$ for some positive integer $k$, it follows that $x^{v} / P^{k}$ belongs to $R_{P}$ and $R_{P}\left[\left(x^{v} / P^{k}\right)^{-1}\right]=$ $\mathbb{Z}\left[x_{i}^{ \pm 1}, 1 / P\right]$; i.e., the larger ring is obtained by inverting $x^{v} / P^{k}$.

We call an element of the form $x^{w} / P$ with $w \in \log P$ a formal monomial in $R_{P}$. (It can happen that $x^{w} / P \in R_{P}$ even if $w \notin \log P-$ e.g., if $w+\log P^{k} \subseteq \log P^{k+1}$ for some $k$. This is not significant for what follows.)

In addition to the obvious facts about $R_{P}$ (it is a commutative, finitely generated hence noetherian - domain), the following results are known:

- $R_{P}=\left\{g / P^{k} \mid g \in \mathbb{Z}[x], \log g \subset \log P^{k}\right\}, R_{P}$ is a partially ordered ring with 1 as an order unit, and it is a dimension group [Handelman 1985, Section I].

- All sums and finite intersections of order ideals are order ideals (this is true for all dimension groups) [Goodearl 1986]. 
- Products of order ideals are order ideals (this is not generally true for dimension groups that are commutative partially ordered domains having 1 as an order unit) [Handelman 1985].

- Every order ideal is an ideal (this is true in every partially ordered commutative ring in which 1 is an order unit) [Handelman 1985, Proposition I.2].

- If $f$ is a formal monomial, then $f R_{P}$ (the ideal generated by $f$ ) is an order ideal [Handelman 1987, Proposition II.2A].

- Every order ideal is the finite sum of ideals, $\sum f_{i} R_{P}$, where $f_{i}$ are formal monomials, and all such sums are order ideals [Handelman 1987, p. 19].

- If $f$ is a formal monomial and $a \in R_{P}$, then $f a \in R_{P}^{+}$implies $a \in R_{P}^{+}$(this follows from the definitions); the conclusion is also true if we replace formal monomial by order unit, a result that is very special for $R_{P}$ [Handelman 1987, Proposition II.5].

- The pure traces are exactly the multiplicative ones (this is true for any partially ordered ring with 1 as an order unit); the pure faithful traces are exactly those of the form $\tau_{r}\left(g / P^{k}\right)=g(r) / P^{k}(r)$, where $r=\left(r_{i}\right)$ is a strictly positive $d$-tuple in $\mathbb{R}^{d}$, and these extend in the obvious way to positive homomorphisms $\tau_{r}: \mathbb{Z}\left[x_{i}^{ \pm 1} ; 1 / P\right] \rightarrow \mathbb{R}$ (warning: although the ring $\mathbb{Z}\left[x_{i}^{ \pm 1} ; 1 / P\right]$ is partially ordered, 1 is not an order unit for it) [Handelman 1985, Theorem III.3].

- The weighted moment map/Legendre transform corresponding to $P$ implements a homeomorphism $\partial_{e} S\left(R_{P}, 1\right) \rightarrow \operatorname{cvx} \log P$ (the latter is the Newton polytope of $P$ ) sending the faithful pure traces onto the interior; unexpectedly, the set of pure traces admits a type of convex structure; in particular, the faces correspond to traces that factor through quotients in a particularly nice way [Handelman 1987, Theorem IV.1].

- In general, $R_{P}$ is not a pure polynomial ring; only rarely does it have unique factorization [Handelman 1987, Theorem A.8A].

Now let us consider the following property of a faithful pure trace $\tau \equiv \tau_{r}$ :

for every nonzero order ideal $I$, we have $\tau_{r}(I)=\tau_{r}\left(R_{P}\right)$.

By Proposition 1.1, this is one of the two necessary conditions for $\tau_{r}$ to be a good trace.

Here $r=\left(r_{i}\right) \in\left(\mathbb{R}^{d}\right)^{++}$as described above. First we note that $\left\{f R_{P}\right\}$ (as $f$ varies over all products of formal monomials) is a generating set of order ideals with order unit (they are given as ring ideals, but in fact are order ideals by the properties above, and every order ideal is a finite sum of these). Necessary and sufficient for (1) to hold is simply that it hold for all ideals of the form $I_{w}=\left(x^{w} / P\right) R_{P}$ (where $w \in \log P$, a finite set). To see this, note that $\tau_{r}\left(I_{w}\right)=\left(r^{w} / P(r)\right) \tau\left(R_{P}\right)$, hence $\tau_{r}\left(I_{w}\right)=\tau_{r}\left(R_{P}\right)$ if and only if $P(r) / r^{w} \in \tau_{r}\left(R_{P}\right)$; thus if this holds for all $w \in \log P$, then each of $P(r) / r^{w}$ belong to $\tau\left(R_{P}\right)$, and hence all their products do; 
this means that for every formal monomial $f, 1 / \tau_{r}(f)$ belongs to $\tau_{r}\left(R_{P}\right)$, hence $\tau_{r}\left(f R_{P}\right)=\tau\left(R_{P}\right)$.

The upshot of this is that $\tau_{r}$ satisfies (1) if and only if for all $w \in \log P$, we have $P(r) / r^{w} \in \tau_{r}\left(R_{P}\right)$. The latter is simply $\mathbb{Z}\left[r^{w} / P(r)\right]_{w \in \log P}$. So we deduce this:

Lemma 3.1. For $r \in\left(\mathbb{R}^{d}\right)^{++}, \tau_{r}$ satisfies (1) if and only if for all $v \in \log P$, $P(r) / r^{v} \in \mathbb{Z}\left[r^{w} / P(r)\right]_{w \in \log P}$.

This is a fairly drastic condition, even when $d=1$ and $P=1+x$ or $2+3 x$.

For $r \in\left(\mathbb{R}^{d}\right)^{++}$and $P \in \mathbb{Z}\left[x_{i}^{ \pm 1}\right]^{+}$, let $R_{r}=\mathbb{Z}\left[\left\{r^{w} / P(r)\right\}_{w \in \log P}\right]$; this is exactly $\tau_{r}\left(R_{P}\right)$, and is a finitely generated unital subring of $\mathbb{R}$. The next lemma says that $r$ satisfies (1) if and only if when we extend $\tau_{r}$ all the way up to $\mathbb{Z}\left[x_{1}^{ \pm 1}, \ldots, x_{d}^{ \pm 1}, P^{-1}\right]$, the image of $\tau_{r}$ does not increase - something we should have expected, in terms of the original definition.

Lemma 3.2. Let $r=\left(r_{i}\right) \in\left(\mathbb{R}^{d}\right)^{++}$and $P \in \mathbb{Z}\left[x_{i}^{ \pm 1}\right]^{+}$be projectively faithful. Then $r$ satisfies (1) if and only if $R_{r}=\mathbb{Z}\left[r_{i}^{ \pm 1} ; P(r)^{-1}\right]$.

Proof. We may construct $R_{P}$ by beginning with $\mathbb{Z}\left[x_{i}^{ \pm 1}\right]$ (the Laurent polynomial ring) instead of $\mathbb{Z}\left[x_{i}\right]$; this is how it was originally constructed in [Handelman 1985; 1987]. By replacing $P$ by $x^{v} P^{t}$ for some $v \in \mathbb{Z}^{d}$ and positive integer $t$ (this has no effect on $R_{P}$, up to order isomorphism), we can arrange for $\mathbf{0}$ to be in the interior of $\operatorname{cvx} \log P$ and in $\log P$. Then $1 / P \in R_{P}$ and we may invert $1 / P$, creating $R_{P}[P]=\mathbb{Z}\left[x_{i}^{ \pm 1} ; P^{-1}\right]$ [Handelman 1987]. Let $I=(1 / P) R_{P} ;$ this is an order ideal [Handelman 1987, p. 19], and $\mathbb{Z}\left[x_{i}^{ \pm 1} ; P^{-1}\right]=\bigcup_{j \in \mathbb{Z}^{+}} P^{j} R_{P}$.

If $r$ satisfies (1) with respect to $P$, then applying it to $I$, we obtain

$$
\tau_{r}(I)=\tau_{r}(1 / P) \tau_{r}(R)=(1 / P(r)) \tau_{r}(R)=(1 / P(r)) R_{r} .
$$

By hypothesis, this is $R_{r}$, so $P(r)$ is a unit in $R_{r}$. Thus $\tau_{r}\left(P^{j} R_{P}\right)=P^{j}(r) R_{r} \subset R_{r}$. Taking the union, we obtain $\tau_{r}\left(\mathbb{Z}\left[x_{i}^{ \pm 1} ; P^{-1}\right]\right) \subseteq R_{r}$, and the reverse inclusion is trivial.

Conversely, suppose $R_{r}=\tau_{r}\left(\mathbb{Z}\left[x_{i}^{ \pm 1} ; P^{-1}\right]\right)$. Then $\tau_{r}\left(x_{i}^{ \pm 1}\right)=r_{i}^{ \pm 1}$ and $\tau_{r}\left(P^{ \pm 1}\right)=$ $P^{ \pm 1}(r)$ belong to $R_{r}$ and are invertible therein. Thus if $f$ is any formal monomial, $\tau_{r}(f)$ is a product of terms of the form $r^{w} / P(r)$, and hence is invertible in $R_{r}$. Thus if $I$ is an order ideal, it contains a formal monomial, and $\tau_{r}(I)$ contains an invertible element in $R_{r}$, and so $\tau_{r}(I)=R_{r}=\tau_{r}\left(R_{P}\right)$. Thus $r$ satisfies (1).

In other words, (1) holds if and only if the range of evaluation at $r$ on $R_{P}$ is the same as the range of the evaluation on the much larger ring $\mathbb{Z}\left[x_{i}^{ \pm 1}, 1 / P\right]$.

Now we consider what (1) means in the special case that $d=1$.

Let $A$ be a unital subring of $\mathbb{C}$, the complexes. A complex number $r$ is integral over $A$ (or $r$ is an A-algebraic integer) if it satisfies a monic polynomial with coefficients from $A$; equivalently, $r \in A\left[r^{-1}\right]$. The number $r$ is an $A$-algebraic unit 
if it satisfies a monic polynomial with coefficients from $A$ whose constant term is invertible in $A$; equivalently, $A[r]=A\left[r^{-1}\right]$. If $A=\mathbb{Z}$, we just write integral (adjective) or algebraic integer (noun). If $A=\mathbb{Q}$, these notions coincide, and we just say $r$ is algebraic. The degree of an integral or algebraic element is the degree of its minimal polynomial (over $A$ ).

Lemma 3.3. Let $P$ be a projectively faithful element of $\mathbb{Z}[x]^{+}$with smallest and largest degree coefficients $a_{0}$ and $a_{k}$ respectively. If $r \in \mathbb{R}^{++}$satisfies (1) with respect to $P$, then there exist nonnegative integers $s$ and $t$ such that $a_{0}^{s} / r$ and $a_{k}^{t} r$ are integral.

Proof. Write $P=a_{0}+\sum_{0<i<k} a_{i} x^{i}+a_{k} x^{k}$, where $a_{i}$ are nonnegative integers (some can be zero, but we still need $\operatorname{gcd}\left(\left\{i \mid a_{i} \neq 0\right\} \cup\{k\}\right)=1$ ). From $P(r) \in \mathbb{Z}\left[\left\{r^{j} / P(r)\right\}_{j \in \log P}\right]$, we deduce an equation of the form $P(r)^{m+1}=p(r)$, where $p \in \mathbb{Z}[x]$ and $\operatorname{deg} p \leq \operatorname{deg} P^{m}=k m$. The leading term of this expression is $a_{k}^{m+1} r^{(m+1) k}$, and so $r$ satisfies a monic polynomial with coefficients from $A=\mathbb{Z}\left[a_{k}^{-1}\right]$. It follows that $a_{k}^{t} r$ is integral for all sufficiently large $s$.

Replacing $P$ by its reversal (also called reciprocal) $\widetilde{P}$ (defined by $\widetilde{P}(x)=$ $\left.P\left(x^{-1}\right) x^{k}\right)$, and redoing the process yields the other form, that $a_{0}^{s} / r$ is integral.

The following is true if we weaken the hypotheses on $P$ to be projectively faithful (instead of requiring all the intermediate coefficients to be strictly positive). The modifications to the proof will muddy an already-complicated but elementary argument; so we just outline it afterwards. We can replace $P$ by any power of itself, without changing anything, so the no-gaps condition is just that the second largest and second smallest terms have nonzero coefficients.

Proposition 3.4. Let $r \in \mathbb{R}^{++}$and $P \in \mathbb{Z}[x]^{+}$be $\sum_{i=0}^{k} a_{i} x^{i}$, where all $a_{i} \neq 0$. Let $a_{0}$ and $a_{k}$ be the coefficients of the least and greatest degree terms in $P$. Let $R_{r}=\mathbb{Z}\left[\left\{r^{i} / P(r)\right\}_{i \in \log P}\right]$. Then the following are equivalent:

(i) $r$ satisfies (1) with respect to $P$.

(ii) There exist nonnegative integers $s$ and $t$ such that both $a_{k}^{s} r$ and $a_{0}^{t} / r$ are algebraic integers.

(iii) $R_{r}=\mathbb{Z}\left[r^{ \pm 1}, P(r)^{ \pm 1}\right]$.

(iv) For all $j \in \log P$, we have $P(r) / r^{j} \in R_{r}$.

Proof. We begin with (ii) implies (iv). Without loss of generality, we may assume $P=a_{0}+\sum_{0<i<k} a_{i} x^{i}+a_{k} x^{k}$.

If $c$ is an algebraic integer, then $\mathbb{Z}[c]$ is free on the $\mathbb{Z}$-basis $\left\{1, c, c^{2}, \ldots, c^{e-1}\right\}$, where $e$ is the degree of $c$ (this is an alternative definition of integrality); in particular, for every positive integer $u$, we can write $c^{u}=\sum_{i=0}^{e-1} b_{i} c^{i}$; in other words, there exists a polynomial $p \in \mathbb{Z}[x]$ of degree at most $e-1$ such that $c^{u}=p(c)$. 
Apply this to $c=a_{k}^{s} r$; for each positive integer $u$, we can write $\left(a_{k}^{s} r\right)^{u}=$ $p_{u}\left(a_{k}^{s} r\right)=q_{u}(r)$, where $\operatorname{deg} q_{u} \leq e-1$. Multiplying this by $r^{u(s-1)}$, we obtain $\left(a_{k} r\right)^{u s}=r^{u(s-1)} q_{u}$; setting $Q_{u}=x^{u(s-1)} q_{u}$, we have $\left(a_{k} r\right)^{u s}=Q_{u}(r)$, where $Q_{u} \in \mathbb{Z}[x]$ and $\operatorname{deg} Q_{u}=u(s-1)+\operatorname{deg} q_{u} \leq u(s-1)+e-1$. Hence (multiplying by an additional $r^{j}$ ), for every $j=0,1,2, \ldots$, we have $Q_{u, j} \in \mathbb{Z}[x]$ such that $\operatorname{deg} Q_{u, j}=u(s-1)+j$ and $\left(a_{k} r\right)^{u s+j}=Q_{u, j}(r)$. We will subsequently choose $u$ to be fairly large.

Now let $N$ be a (large) positive integer, and consider the $k$ leading coefficients of $P^{N}$, that is, the coefficients of the terms $x^{k N}, x^{k N-1}, x^{k N-2}, \ldots, x^{k N-k+1}$. They are respectively divisible by $a_{k}^{N}, a_{k}^{N-1}, \ldots, a_{k}^{N-k+1}$ (as is trivially easy to see). Hence we may find integers $b_{i}$ (with $b_{0}=1$ ) such that

$$
P^{N}-\sum_{i=0}^{k-1}\left(a_{k} x\right)^{N-i} x^{N(k-1)} b_{i}:=G
$$

is a polynomial of degree at most $N k-k$. Assume (as we may) that $N-k=u s$ for some integer $u$. Replace each $\left(a_{k} x^{N-i}\right)$ by $Q_{u, k-i}$; this has no effect on the value at $r$. Setting $H=\sum_{i=0}^{k-1} b_{i} Q_{u, k-i} x^{N(k-1)}$, we have $P^{N}(r)=(G+H)(r)$. Then

$$
\begin{aligned}
\operatorname{deg}(G+H) & \leq \max \{\operatorname{deg} G, \operatorname{deg} H\} \\
& \leq \max \left\{N k-k, \max _{i}\left\{\operatorname{deg} Q_{u, k-i}+N k-N\right\}\right\} \\
& \leq \max \{N k-k, u(s-1)+e-1+N k-N\} \\
& =\max \{N k-k, N k-N+e-1+N-k-u\} \\
& \leq \max \{N k-k, N k-k-u+e-1\} .
\end{aligned}
$$

We can choose $u \geq e-1$ at the outset, and so guarantee that $\operatorname{deg}(G+H) \leq N k-k$. Thus $P(r)=(G+H)(r) / P^{N-1}(r)$. For every $0 \leq i \leq k$, we have $r^{i} / P(r) \in R_{r}$, and since $\operatorname{deg}(G+H) \leq N k-k=\operatorname{deg} P^{N-1}$, we obtain $P(r) \in R_{r}$.

Now form the reversal of $P$, given by $\widetilde{P}(x)=P\left(x^{-1}\right) x^{k}$; this reverses the roles of $a_{k}$ and $a_{0}$, and the same process (using $a_{0}^{t} / r$ being integral) yields, after translating back, $P(r) / r^{k} \in R_{r}$. From $P(r) \in R_{r}$, we obtain $r^{i}=\left(r^{i} / P(r)\right) \cdot P(r) \in R_{r}$ for $i \in \log P$, and thus for all $i \geq 0$. Since $P(r) / r^{k} \in R_{r}$, we deduce $r^{-k} \in R_{r}$, hence $r^{-j} \in R_{r}$ for all $j \geq 0$; thus $P(r) / r^{j} \in R_{r}$.

Now (i) implies (ii) was done in the previous lemma, and the equivalence of (i), (iii), and (iv) follows from the general results preceding this.

To prove the result when $P$ is only projectively faithful, we can still write $P=$ $a_{0}+\sum_{1 \leq i \leq k-1} a_{i} x^{i}+a_{k} x^{k}$, where gcd $\left\{i \mid a_{i} \neq 0\right\}=1$ (equivalent after translation to projective faithfulness). Then it is elementary, and presumably well-known, that there exists $M$ such that for all $N$, we have $\left(P^{N}, x^{i}\right) \neq 0$ if $M<i<k N-M$. Now in the construction above, make sure that when the multiplications by powers 
of $r$ take place, the exponent lands in the interval where all the coefficients are guaranteed to be nonzero (we are of course free to take arbitrary large powers of $P$ ).

A strange consequence is that when the hypotheses on $P$ are satisfied, the set of $r$ such that $\tau_{r}$ satisfies (1) is closed under multiplication; this follows immediately from (ii), but not obviously from any of the other equivalent properties.

Multiplicativity does not appear to extend to more than one variable. For example, if $P=2+3 x+5 y$, and we restrict to $r=(m, n)$ with positive integer coordinates, it is tedious but routine to see that $\tau_{r}$ satisfies (1) with respect to $P$ if and only if for all primes $p$ and $q$,

$$
p|m \Longrightarrow p|(2+5 n) \text { and } q|n \Longrightarrow q|(2+3 m) .
$$

For example, $(7,1),(3,11),\left(2^{i}, 2^{j}\right)$ (where both $\left.i, j>0\right)$ satisfy these conditions, but $(14,2)$ does not. There may be another, more appropriate, notion of multiplication with respect to which the set is closed.

Another general property concerns approximate divisibility. Let $K=\operatorname{cvx} \log P$; this is a compact convex polytope. Let $e \in K$ be an extreme point (we do not use the usual term, vertex, because this might be confused with lattice point); then $v \in \log P$, and there is a pure trace $\sigma^{v}$ associated with $v$, given by $\sigma^{v}\left(g / P^{k}\right)=$ $\left(g, x^{k v}\right) /\left(P, x^{v}\right)^{k}$ (this can also be obtained as the limit along a path of $\left.\tau_{r}\right)$, via l'Hôpital's rule, as in [Handelman 1985, Section III] (especially just before III.3).

Since every order ideal of $R_{P}$ is of the form $\sum f_{i} R_{P}$ (finite sum), if we assume that $R_{P}$ is approximately divisible, then $R_{P}$ is nearly divisible. Thus every order ideal has its own order unit and is approximately divisible. If $\tau$ is faithful, then $\tau(I \cap J) \neq 0$ (no finite intersections of order ideals can be zero since they are also ideals in a domain), and $I \cap J$ is itself approximately divisible, hence $\tau(I \cap J)$ is dense in $\mathbb{R}$. Thus for any faithful trace that is order unit good for $R_{P}$, its restriction to any nonzero order ideal is also order unit good.

Thus we have the following.

Lemma 3.5. The ordered ring $R_{P}$ is approximately divisible if and only if for all extreme points $v$ of $K=\operatorname{cvx} \log P$, we have $\left(P, x^{v}\right)>1$.

Lemma 3.6. Let $P=\sum \lambda_{w} x^{w} \in \mathbb{Z}\left[x_{i}^{ \pm 1}\right]^{+}$with $\left(P, x^{v}\right)>1$ for all extreme points of $K=\operatorname{cvx} \log P$.

(a) Then $R_{P}$ is nearly divisible

(b) If $\tau$ is a faithful trace that is order unit good for $R_{P}$, then its restriction to any nonzero ideal is order unit good for that ideal.

If we replace $R_{P}$ by $S_{P}:=R_{P} \otimes \mathbb{Q}=\mathbb{Q}\left[x^{w} / P\right]$, then it is divisible, which is of course stronger than nearly divisible, so that (a) holds automatically (without the hypothesis on the coefficients at extreme points), and (b) also holds by the same arguments. 
Proposition 3.7. Let $r=\left(r_{i}\right) \in\left(\mathbb{R}^{d}\right)^{++}$, and let $P \in \mathbb{Z}\left[x_{i}^{ \pm 1}\right]^{+}$be projectively faithful.

(a) The pure trace $\tau_{r}$ on $R_{P}$ is good if and only if

(i) $\tau_{r}$ is order unit good for $R_{P}$ and

(ii) for all $v \in \log P$, we have $P(r) / r^{v} \in \mathbb{Z}\left[r^{w} / P(r)\right]_{w \in \log P \text {. }}$

(b) The pure trace $\tau_{r}$ on $S_{P}$ is good if and only if

(i) $\tau_{r}$ is order unit good for $R_{P}$.

Remark. Note the absence of (ii) from (b), and the appearance of $R_{P}$ in (b)(i). It is known (along the same lines as in [BeH 2014, Proposition 5.10]), that if $\tau_{r}$ is order unit good (for either coefficient ring), then each $r_{i}$ is algebraic. Since $\mathbb{Q}\left[r_{1}, \ldots, r_{d}\right]$ is thus a field, (ii) is redundant in (b).

Proof. We show that if $\tau_{r}$ is order unit good (which means that the closure of the image of $\operatorname{ker} \tau_{r}$ in Aff $S(R, 1)$ is exactly $\left.\tau_{r}^{\perp}=\left\{h \in \operatorname{Aff} S(R, 1) \mid h\left(\tau_{r}\right)=0\right\}\right)$, then its restriction to any order ideal is also order unit good. It suffices to do this for $I=f R_{P}$, where $f$ is a formal monomial.

The map $R_{P} \rightarrow f R_{P}$, given by $r \mapsto f r$, is an order-isomorphism of $R_{P}$ modules (this of course uses the fact that $f r \geq 0$ in $R_{P}$ entails $r \geq 0$ ). Using $f$ as an order unit for $I$, the map on traces $\tau \mapsto \tau / \tau(f)$ (restricted to those $\tau$ such that $\tau(f) \neq 0$ ) sends $\tau_{r} \rightarrow \tau_{r} / \tau_{r}(f)=\tau^{\prime}$, and $\operatorname{ker} \tau^{\prime}=\operatorname{ker} \tau_{r} \cap f R_{P}=f \cdot \operatorname{ker} \tau_{r}$ (since $\left.f(r) \neq 0\right)$. The map between $R_{P}$ modules induces an affine homeomorphism between $S\left(R_{P}, 1\right)$ and $S(I, f)$, sending $\tau_{r}$ to $\tau^{\prime}$, and it easily follows that $\tau^{\prime}$ is order unit good. But $\tau^{\prime}$ is just the normalization of $\tau \mid I$, hence the latter is order unit good.

The rest follows from the preceding results.

In one variable, we can show that $\tau_{r}$ is order unit good if and only if none of the algebraic conjugates of $r$ (except itself) are positive real. In more than one variable, the situation is far more complicated, and there is no decisive theorem (yet).

Example. Let $d=1$ and $P=1+x$; then we can rewrite $R_{P}=\mathbb{Z}[1 / P, x / P]=$ $\mathbb{Z}[1-X, X]$, where $X=x /(1+x)$, and the positive cone translates to $\langle X, 1-X\rangle$. This goes back to Renault [1980]. The translation, however, obscures some of the features, as we will see. First, $R_{P}$ has two discrete pure traces, $\tau_{0}=\sigma^{0}$ and $\tau_{\infty}=\sigma^{1}$ ( 0 and 1 are the extreme points of the convex set cvx $\log P=[0,1]$ ), so it is not approximately divisible. However, it is interesting to calculate the condition that $\tau_{r}(I)=\tau_{r}\left(R_{P}\right)$ for all nonzero order ideals.

By Proposition 3.7 above, this amounts to $1+r, 1+1 / r \in \mathbb{Z}[1 /(1+r), r /(1+r)]$; as $r /(1+r)=1-1 /(1+r)$, the condition (1) is equivalent to $1+r^{ \pm 1} \in \mathbb{Z}[1 /(1+r)]$. Now for a real number $s$, the condition $s \in \mathbb{Z}[1 / s]$ is equivalent to $s$ being an algebraic integer (that is, satisfying a monic integer polynomial). Hence we infer that if (1) holds for $\tau_{r}$, then $r$ has to be an algebraic unit (that is, not only is its minimal 
polynomial over the integers monic, but the constant term must be \pm 1 as well). Conversely, if $r$ is an algebraic unit, then the desired membership property holds.

We conclude that $\tau_{r}$ satisfies (1) if and only if $r$ is an algebraic unit.

In particular, if $r$ is an integer, then $\tau_{r}$ satisfies (1) if and only if $r=1$ (we are restricting ourselves to actual traces, hence excluding negative values for $r$ ).

The translation, $X=x /(1+x)$ converts $r$ to $r /(1+r)$; then of course $\tau(X)$ is a fractional linear transformation of an algebraic unit, but this characterization is not as pleasant as the pretranslation version.

Let $V \subset \mathbb{C}^{d}$. For $A$ a subring of $\mathbb{C}$, define $I_{A}(V)$ to be the ideal in the polynomial ring $A\left[x_{1}, \ldots, x_{d}\right]$ consisting of polynomials that vanish at all points of $V$. Given an ideal $I$ of $A\left[x_{1}, \ldots, x_{d}\right]$, define $Z_{A}(I)$ to be the common zero set (in $\mathbb{C}^{d}$ ) of all elements of $I$. The variety generated by $V$ over $A$ is simply $Z_{A} I_{A}(V)$. If $A=\mathbb{Z}$, we drop the subscript.

We say $r=\left(r_{i}\right) \in\left(\mathbb{R}^{d}\right)^{++}$is really isolated if $Z I(\{r\}) \cap\left(\mathbb{R}^{d}\right)^{++}=\{r\}$. For example, if $d=1$, then $r$ is really isolated if $r$ is algebraic and all algebraic conjugates of $r$ other than $r$ itself are not positive real. In general, $r$ is really isolated means that the slice of the variety generated by $r$ (or more simply, the Zariski closure of $\{r\})$ by the positive orthant contains only $r$.

The argument in [BeH 2014, Proposition 5.10] shows that if $r$ is really isolated (or more generally, $\{r\}$ is an isolated point in $\left(\mathbb{R}^{d}\right)^{++} \cap Z I(\{r\})$ ), then all of its coordinates are algebraic (there is an assumption in [BeH 2014] concerning interior points which is automatic here). We remind the reader that we have assumed that $P$ is projectively faithful, which implies in particular, that its Newton polytope contains a $d$-ball.

The condition that $r$ be really isolated appears in [BeH 2014, Examples 5 and 10], for which the relevant dimension groups are remotely related to the ones appearing here.

Proposition 3.8. Suppose $R_{P}$ is approximately divisible, and $\tau$ is a pure faithful trace. Then:

(a) $\tau$ is an order unit good trace of $R_{P}$ if and only if $\tau=\tau_{r}$, where $r \in\left(\mathbb{R}^{d}\right)^{++}$is really isolated.

(b) $\tau_{r}$ is a good trace of $R_{P}$ if and only if $r$ is really isolated and for all $v \in \log P$, we have $P(r) / r^{v} \in \mathbb{Z}\left[\left\{r^{w} / P(r)\right\}_{w \in \log P}\right]$.

(c) $\tau_{r}$ is a good trace of $R_{P} \otimes \mathbb{Q}$ if and only if $r$ is really isolated.

Proof. Every pure faithful trace of $R_{P}$ is of the form $\tau_{r}$ for (a unique) $r$ in the positive orthant.

If $r$ is not really isolated, then there exists $r^{\prime} \in\left(\mathbb{R}^{d}\right)^{++}$such that every polynomial that vanishes at $r$ also vanishes at $r^{\prime}$. Suppose $a:=g / P^{k} \in R_{P}$; we may assume $\log g \subseteq \log P^{k}$. If $\tau_{r}(a)=0$, then $g(r)=0$, hence $g\left(r^{\prime}\right)=0$, whence $\tau_{r^{\prime}}(a)=0$; 
thus with $\sigma=\tau_{r^{\prime}}$, we have $\sigma \in \partial_{e} S(R, 1) \backslash\left\{\tau_{r}\right\}$ such that $\sigma \mid \operatorname{ker} \tau_{r} \equiv 0$. Hence $\tau_{r}$ is not order unit good. The same of course applies with $R_{P} \otimes \mathbb{Q}$ in place of $R_{P}$.

Conversely, suppose that $r$ is really isolated, but there exists $\sigma \in \partial_{e} S(R, 1) \backslash\left\{\tau_{r}\right\}$ such that $\sigma \mid \operatorname{ker} \tau_{r}=0$. Then $\sigma$ cannot be faithful (as otherwise, $\sigma=\tau_{r^{\prime}}$ for some $r^{\prime} \in\left(\mathbb{R}^{d}\right)^{++}$, and $\left.r^{\prime} \in Z I(\{r\})\right)$. Consider $S=R_{P} \otimes \mathbb{Q}$, and let $T_{r}, \Sigma$ be the extension to $S$ of $\tau_{r}$ and $\Sigma$ (both extend, since the ranges are torsion-free abelian groups). Then $T_{r}(S)=\mathbb{Q}\left[r^{w} / P(r)\right]$, which is a field (since the coordinates are algebraic, so are all the $\left.r^{w} / P(r)\right)$. Then $\operatorname{ker} T_{r}$ is a field, so $\operatorname{ker} T_{r}$ is a maximal ideal. Also, $\operatorname{ker} T_{r} \cap R_{P}=\operatorname{ker} \tau_{r}$ and $\operatorname{ker} \Sigma \cap R_{P}=\sigma$. If $\operatorname{ker} \tau_{r} \subseteq \operatorname{ker} \sigma$, then $\operatorname{ker} T_{r} \subset \operatorname{ker} \Sigma$, but maximality of $\operatorname{ker} T_{r}$ implies $\operatorname{ker} T_{r}=\operatorname{ker} \Sigma$, and thus $\operatorname{ker} \tau_{r}=\operatorname{ker} \sigma$. However, since $\sigma$ is not faithful, $\operatorname{ker} \sigma$ contains a positive nonzero element of $R_{P}$, whereas $\operatorname{ker} \tau_{r}$ does not, a contradiction.

Hence if $r$ is really isolated, then $\sigma \in \partial_{e} S\left(R_{P}, 1\right) \backslash\left\{\tau_{r}\right\}$ implies $\sigma\left(\operatorname{ker} \tau_{r}\right) \neq 0$, and by Lemma 1.7 above, this implies $\tau_{r}$ is order unit good. The same of course applies to $T_{r}$ as a trace on $S_{P}$. This yields (a), and contributes to (c).

Part (b) now follows from preceding results in this section.

Part (c) comes from $\mathbb{Q}\left[r^{w} / P(r)\right]$ being a field (which in turn arises because the coordinates of $r$ are algebraic), so that condition (1) is automatic.

A particular consequence is that the set of good pure faithful traces of $S_{P}=$ $R_{P} \otimes \mathbb{Q}$ is the same for all choices (with $d$ fixed) of faithfully projective $P \in \mathbb{Z}\left[x_{i}\right]^{+}$ (or $P \in \mathbb{Q}\left[x_{i}\right]^{+}$), whereas for $R_{P}$, there is dependence on $P$.

When $d=1$, the conditions for $\tau_{r}$ to be good are precisely that no distinct algebraic conjugate of $r$ be positive and the integrality condition, (ii), of Proposition 3.4.

Example. Let $d=1$ and $P=2+3 x$. By Proposition 3.4, the positive real number $r$ satisfies (1) if and only if there exists $s$ such that both $2^{s} / r$ and $3^{s} r$ are integral. Let $K=\mathbb{Q}(r)$, and $\mathbb{Z}_{K}$ denote the ring of integers in $K$. The fractional ideal $r \mathbb{Z}_{K}$ factors as $\prod \mathcal{P}_{i} / \prod \mathcal{Q}_{j}$ (where $\mathcal{P}_{i}$ and $\mathcal{Q}_{j}$ are prime ideals in $\mathbb{Z}_{K}$, and we allow repetitions; the products might also be over the empty set). The intersections $\mathcal{P}_{i} \cap \mathbb{Z}$ and $\mathcal{Q}_{j} \cap \mathbb{Z}$ determine primes in $\mathbb{Z}$, denoted respectively $p_{i}$ and $q_{j}$. Then (1) is equivalent to $p_{i}=2$ and $q_{j}=3$ for all $i$ and $j$. Hence $\tau_{r}$ is good for $R_{P}$ if and only if no nonidentity algebraic conjugate is positive and the prime factorization of the fractional ideal $r \mathbb{Z}_{K}$ consists of primes sitting over 2 in the numerator and over 3 in the denominator.

In this section, we have restricted ourselves to pure faithful traces; this is a technical convenience. By the comment after Proposition 1.1, we can factor out the largest order ideal contained in the kernel of a trace, and in the case that the dimension group is $R_{P}$, these correspond to quotients corresponding to faces of the Newton polytope [Handelman 1985, Section VII]. This amounts to a reduction to a lower dimensional lattice and vector space, that is, a polynomial in fewer variables. 
There are related naturally occurring classes of dimension groups whose pure traces can be similarly analyzed. For example, for the matrix-valued random walks appearing in [Handelman 2009], in nondegenerate cases, the pure faithful traces are similarly parameterized by the positive orthant (the nonfaithful traces are generically terrible, but can be analyzed in reasonable cases). An example appears in [Petersen 2012], where very specific local limit asymptotics were used to derive the one-parameter family (indexed by the unit interval) of pure traces. In fact, that random walk can be represented as $M=\left(\begin{array}{rr}1+x & x \\ 1 & 0\end{array}\right)$, and in this very simple case, via [Handelman 2009], we can write down the pure traces parameterized by $[0, \infty]$ (the endpoints corresponding to the two nonfaithful pure traces) via the large eigenvalue function. Alternatively (in the notation of [Handelman 2009]), it is elementary that $(1+x) \widehat{M}^{-1}$ is an order unit in $E_{b}\left(G_{M}\right)$, so on setting $P=1+x$, we can view $M / P$ as a matrix with entries in $R_{P}$ without changing the pure trace space. This yields a parameterization of the pure traces by those of $R_{P}$ (again via the large eigenvalue function, an algebraic function), which are indexed by the unit interval.

\section{Direct sums and goodness}

For (noncyclic) simple dimension groups, there is a notion of direct sum (corresponding to coproduct; see [BeH 2014, Appendix B] for a discussion). This actually extends to nearly divisible dimension groups. Let $G$ and $H$ be dimension groups. Form the group direct sum $K=G \oplus H$, and impose on it the strict ordering given by the positive cone

$$
K^{+}:=\left\{(g, h) \mid g \in G^{+} \backslash\{0\} \text { and } h \in H^{+} \backslash\{0\}\right\} \cup\{(0,0)\} .
$$

We see immediately that $K$ is an unperforated partially ordered group; we denote it by $G \oplus_{s} H$, although we frequently suppress the subscript $s$. In general, $K$ need not be a dimension group (as a simple example, if $G$ is simple and $H=\mathbb{Z}$, then $K$ is a simple partially ordered abelian group with a discrete trace, and hence cannot be a dimension group [Goodearl 1986, Proposition 4.22]).

A partially ordered abelian group $G$ is prime if the intersection of any two nonzero order ideals contains a nonzero positive element; for dimension groups, this definition simplifies to "the intersection of any nonzero order ideals is nonzero". Here is a natural generalization of [Effros et al. 1980, Corollary 1.2].

Lemma 4.1. Let $G$ and $H$ be dimension groups. Then the strict direct sum $K=$ $G \oplus_{s} H$ is a dimension group if and only if both of the following conditions hold:

(a) Both $G$ and $H$ are prime.

(b) Both $G$ and $H$ are nearly divisible. 
Proof. Assume (a) and (b). The unperforation and directedness of $K$ are trivial, so it suffices to prove Riesz decomposition. Suppose $0 \leq a \leq b+c$, where $a=(g, h)$, $b=(e, f)$, and $c=(k, l)$ and all of $a, b, c$ are in $K^{+} \backslash\{0\}$ (if any of $a, b$, or $c$ is zero, the decomposition is immediate). This entails all of $g, h, e, f, k, l$ are nonzero positive elements of their respective groups, and moreover, that either $a=b+c$ (in which case, the decomposition condition is satisfied) or both $g<e+k$ and $h<f+l$ in their respective groups. Assume the latter.

By Riesz decomposition in $G$, there exist $0 \leq e_{1} \leq e$ and $0 \leq k_{1} \leq k$ such that $g=e_{1}+k_{1}$. If $e=e_{1}$, then $k_{1}<k$, and the intersection of the order ideals $\langle e\rangle \cap\left\langle k-k_{1}\right\rangle$ is thus nonzero, and contains a nonzero positive element, $z$. The order ideal $\langle z\rangle$ has $z$ as an order unit, hence (by (b)) is approximately divisible, and it follows immediately that there exists a positive, nonzero $x$ such that $x<e, k-k_{1}$. Then we can write $g=\left(e_{1}-x\right)+\left(k_{1}+x\right)$. Now $e-\left(e_{1}-x\right)>0$, and $k-\left(k_{1}+x\right)>0$.

Now suppose that $e_{1}=0$, so that $g=k_{1}$. By the same procedure as in the previous paragraph, there exists a nonzero positive $x$ such that $x<e, k_{1}$, so we can write $g=x+\left(k_{1}-x\right)$, with $x<e$ and $k_{1}-x<k$.

This leaves the case that $0<e_{1}<e$. If $k_{1}$ is zero or $k$, we reverse the roles of $e$ and $k$ and apply the preceding, so that in all cases, we can find nonzero positive $x_{i} \in G$ such that $g=x_{1}+x_{2}$, with $0<x_{1}<e$ and $0<x_{2}<k$.

By applying the preceding to $H$ in place of $G$, we obtain $y_{i} \in H$ such that $h=y_{1}+y_{2}$ with $0<y_{1}<f$ and $0<y_{2}<l$. Then $g=\left(x_{1}, y_{1}\right)+\left(x_{2}, y_{2}\right)$ is the desired decomposition.

Conversely, suppose that $K$ satisfies Riesz decomposition. If $H$ were not prime, we could find nonzero $y, z$ in $H^{+}$such that $0 \leq h \leq y, z$ implies $h=0$. Consider, for $g \in G^{+} \backslash\{0\},(5 g, y) \leq(3 g, y)+(3 g, z)$ : this holds in $K$; hence if Riesz decomposition applies, we can write $(5 g, y)=\left(3 g, y_{1}\right)+\left(3 g, z_{1}\right)$ with the latter two terms in $K^{+}$, and at the very least $y_{1} \leq y$ and $z_{i} \leq z$. In particular $y=y_{1}+z_{1}$, but since $z_{1} \leq y, z$, we have $z_{1}=0$, so that $y_{1}=y$. But this entails $(3 g, 0)$ is in the positive cone of $K$, which is a contradiction, since $g>0$. Now the same argument applies to $G$, so both have to be prime.

Suppose that $K$ is a dimension group; we can also assume that $G$ admits an order unit. Now assume that $H$ is not nearly divisible. Then $H$ admits an order ideal with its own order unit, $(I, w)$, that has a discrete pure trace; call it $\tau$. Then $\operatorname{ker} \tau$ is a maximal order ideal of $H$; call it $T$. Since $G$ has an order unit, it has a maximal order ideal, $J$. Consider $J \oplus_{s} T$. This is an order ideal of $K$, and it is easy to verify that $K /(J \oplus T)$ is order isomorphic to $K^{\prime}:=(G / J) \oplus_{s} \mathbb{Z}$. Since both pieces are simple, $K^{\prime}$ is simple, but admits a discrete trace (projection on the second coordinate). Thus $K^{\prime}$ is not a dimension group. On the other hand, since $K$ is a dimension group, and the quotient of it by an order ideal is also a dimension group, we have a contradiction. Hence $H$ must be nearly divisible. The same applies with $H$ replaced by $G$. 
If $K=G \oplus_{s} H$, and $\sigma$ and $\tau$ are traces on $G$ and $H$ respectively, we consider the possibility that $\phi:=\sigma \oplus \tau$ (defined by $(g, h) \mapsto \sigma(g)+\tau(h))$ is good or order unit good. This turns out to be surprisingly interesting. Iteration of this process yields some weird examples.

Lemma 4.2 (a consequence of the method of proof of [BeH 2014, Proposition 1.7]). Suppose $(K, w)$ is an approximately divisible dimension group with order unit, and $\phi$ is an order unit good trace. Then whenever $a \in G, b \in G^{++}$and $0<\phi(a)<\phi(b)$, for all $\epsilon>0$, there exists $a^{\prime} \in[0, b]$ such that $\phi\left(a^{\prime}\right)=a$ and $\left\|\hat{a}^{\prime}-\hat{b} \sigma(a) / \sigma(b)\right\|<\epsilon$.

Proof. Approximate divisibility implies density of $G$ in $\operatorname{Aff} S(G, u)$. Set $j=$ $\sigma(b) \hat{b} / \sigma(a)$, so that $j(\sigma)=\sigma(a)$ and $\inf j=\sigma(a) \sigma(b)^{-1} \inf \hat{b}$. There exists $g_{n} \in G$ such that $\hat{g}_{n} \rightarrow j$ uniformly. If for infinitely many $n$, we have $g_{n}(\sigma)=\sigma(a)$, we are done (taking large enough $n$ ). Otherwise, select $\sigma(a)(\sigma(b) 2)^{-1}$ inf $b>\epsilon>0$ and $\left\|\hat{g}_{n}-j\right\|<\epsilon$. Then $\left|\sigma\left(g_{n}\right)-\sigma(a)\right|<\epsilon$ provided $n$ is sufficiently large; if $\sigma\left(g_{n}\right)>\sigma(a)$, set $c_{n}=g_{n}-a$. There exists an order unit $z_{n}$ such that $0<\sigma\left(c_{n}\right) \mathbf{1}<$ $\hat{z}_{n}<2 \epsilon$. By order unit goodness, there exists $v_{n} \ll z_{n}$ such that $\sigma\left(c_{n}\right)=\sigma\left(v_{n}\right)$, and of course, $\left\|v_{n}\right\| \leq\left\|\hat{z}_{n}\right\|<2 \epsilon$. Then $g_{n}-v_{n}$ has image within $3 \epsilon$ of $j$, and it is easy to check that $g_{n}-v_{n}$ is strictly positive, and hence is an order unit.

If instead, $\sigma(a)>\sigma\left(c_{n}\right)$ for infinitely many $n$, we obtain a corresponding $c_{n}=g_{n}-a$ and $v_{n} \ll z_{n}$, and this time, $g_{n}+v_{n}$ has all the right properties. In both cases, by taking $n$ sufficiently large, we make the error terms go to zero, and hence obtain the $a^{\prime}$ as one of $g_{n} \pm v_{n}$.

In the following, the function $\psi$ need not be a group homomorphism.

Lemma 4.3. Suppose $G$ and $H$ are nearly divisible dimension groups, each with order unit, and respective trace $\sigma$ and $\tau$. Let $K=G \oplus H$ with the strict ordering, and suppose that the trace on $K, \phi:=\sigma \oplus \tau$, is order unit good. Then provided the following condition holds, $\sigma$ is order unit good as a trace on $G$ :

- There exists a function $\psi: \tau^{-1}(\sigma(G) \cap \tau(H)) \rightarrow \sigma^{-1}(\sigma(G) \cap \tau(H))$ that is pseudonorm continuous with the additional property that $\sigma \psi=\tau$.

Remark. As we will see below, without the weird extra condition, the result fails.

Proof. Select an order unit $b$ in $G$, and $a$ in $G$ such that $0<\sigma(a)<\sigma(b)$. As $H$ is approximately divisible, there is a sequence of order units $\left(h_{n}\right)$ in $H$ such that $h_{n} \rightarrow 0$ (with respect to the pseudonorm topology on $H$; equivalently, as functions on $S(H, v), \hat{h}_{n}$ converges uniformly to zero). There also exists $\delta$ in $G$ such that $\sigma(b-a) / 4<\hat{\delta}<\min \left\{\sigma(b-a) / 2, \inf _{\theta \in S(G, u)} \theta(b) / 2\right\}$ uniformly on $S(G, u)$. Then $B_{n}:=\left(b-\delta, h_{n}\right)$ are order units of $G \oplus H$, and $\phi(a, 0)<\phi\left(B_{n}\right)=$ $\sigma(b)-\sigma(\delta)+\tau\left(h_{n}\right)$.

Since $\phi$ is order unit good and each $B_{n}$ is an order unit, there exist $\left(a_{n}, z_{n}\right)$ such that $0 \ll a_{n} \ll b-\delta$ and $0 \ll z_{n} \ll h_{n}$, with $\phi\left(\left(a_{n}, z_{n}\right)\right)=\sigma(a)$, and by the previous 
lemma, $\inf _{S(G, u)} \hat{a}_{n}$ is bounded below (as $n \rightarrow \infty$ ); in particular, $\left\|z_{n}\right\|_{H} \rightarrow 0$ and $\sigma\left(a_{n}\right)+\tau\left(z_{n}\right)=\sigma(a)$. Thus $z_{n} \in \tau^{-1}(\sigma(G) \cap \tau(H))$, so we may consider the sequence $\psi\left(z_{n}\right) \in \sigma^{-1}(\sigma(G) \cap \tau(H))$. Since $\psi$ is pseudonorm continuous, $\widehat{\psi\left(z_{n}\right)} \rightarrow 0$ uniformly on $S(G, u)$.

Consider $a_{n}+\psi\left(z_{n}\right)$; its value at $\sigma$ is $\sigma\left(a_{n}\right)+\sigma\left(\psi\left(z_{n}\right)\right)=\sigma\left(a_{n}\right)+\tau\left(z_{n}\right)=\sigma(a)$. If we choose $n$ sufficiently large so that $\left\|\widehat{\psi\left(z_{n}\right)}\right\|<\inf \delta$, then $a_{n}+\psi\left(z_{n}\right) \ll b-\delta+\delta=b$. In addition, we can also choose $n$ sufficiently large so that inf $\overline{\psi\left(z_{n}\right)}>-\inf _{S(G, u)} \hat{a}_{n}$, by the uniform boundedness below of the $a_{n}$ (there is no guarantee that $\psi\left(z_{n}\right)$ is positive). Then $a_{n}+\psi\left(z_{n}\right)$ is an order unit in the interval $[0, b]$ and we are done.

One advantage of not requiring normalization of $\sigma$ and $\tau$ is that we can replace them by any positive scalar multiples in testing for order unit goodness of $\lambda \sigma \oplus \mu \tau$; the first hypotheses are unchanged, but the second translates to density of $(\lambda \sigma(G)) \cap(\mu \tau(G))$ in $\mathbb{R}$. In the following, we cannot apply earlier results directly, since $G \oplus 0$ is not an order ideal of $G \oplus H$ (strict ordering).

Lemma 4.4. Suppose that $\sigma$ is a trace on $G, \tau$ is a trace on $H$, and $\sigma \oplus \tau=\phi$ is order unit good for $K=G \oplus H$ with the strict ordering, and moreover assume that each of $G$ and $H$ is nearly divisible. Then $\sigma(G) \cap \tau(H)$ is dense in $\mathbb{R}$.

Proof. We use the characterization of order unit good traces on approximately divisible dimension groups; namely $\operatorname{ker} \phi$ has dense image in $\phi^{\vdash}$ [BeH 2014, Proposition 1.7].

Suppose the intersection is not dense; then there exists a real $\delta \geq 0$ such that $\sigma(G) \cap \tau(H)=\delta \mathbb{Z}$. We have that $\operatorname{ker} \phi$ has dense range in Aff $S(K,(u, v))=$ Aff $S(G, u) \times$ Aff $S(H, v)$. But

$$
\operatorname{ker} \phi=\{(g, h) \in G \oplus H \mid \sigma(g)=-\tau(h)\} .
$$

If $\delta=0$, then $\operatorname{ker} \phi=\operatorname{ker} \sigma \oplus \operatorname{ker} \tau$ (since $\sigma(g)=-\tau(h)$ implies $\sigma(g) \in$ $\tau(H) \cap \sigma(H)$, and hence is zero). The image of $\operatorname{ker} \phi$ is then contained in $\sigma^{\vdash} \times \tau^{\vdash}$, which is closed and of codimension two in Aff $S(K,(u, v))$, and so ker $\phi$ cannot be dense in $\phi^{\vdash}$ (which has codimension one), hence $\phi$ cannot be order unit good.

If $\delta \neq 0$, select $g$ and $h$ in $G$ and $H$ respectively such that $\sigma(g)=\delta=\tau(h)$. Then it is easy to see that $\operatorname{ker} \phi=(\operatorname{ker} \sigma \oplus \operatorname{ker} \tau)+(g,-h) \mathbb{Z}$, and then its range is contained in $\left(\sigma^{\vdash} \times \tau^{\vdash}\right)+(\hat{g},-\hat{h}) \mathbb{Z}$. However, the latter is closed (easy to see), and so the image of $\operatorname{ker} \phi$ is contained in a proper closed subspace (with a discrete direct summand) of $\phi^{\vdash}$; hence in this case as well, $\phi$ is not order unit good.

Now we want to determine when $\sigma \oplus \tau$ is good or order unit good. Let $\pi_{G}: G \oplus H \rightarrow G$ and $\pi_{H}: G \oplus H \rightarrow H$ be the obvious projection maps. Unlike the inclusions $G, H \rightarrow G \oplus H$, these are order-preserving. First, consider $\sigma \circ \pi_{G}: \operatorname{ker} \phi \rightarrow \sigma(G) \cap \tau(H) \subseteq \mathbb{R}$. The kernel is exactly $\operatorname{ker} \sigma \oplus \operatorname{ker} \tau$; we also note that $\sigma$ extends to a map $\Sigma: \phi^{\vdash} \rightarrow \mathbb{R}$ (sending $(j, l)$ to $j(\sigma)$ ), the kernel of which is 
$\sigma^{\vdash} \times \tau^{\vdash}$. Via the identification of Aff $S(K,(u, v))$ with $\operatorname{Aff} S(G, u) \times \operatorname{Aff} S(H, v)$, we have the following diagram:

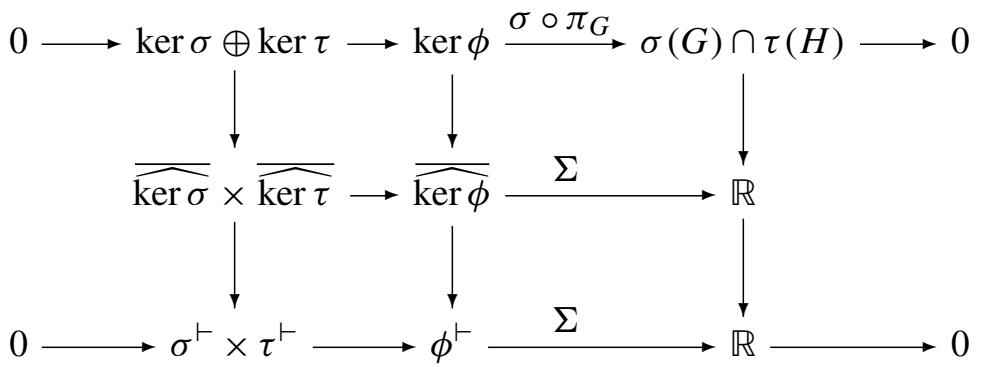

The long horizontal overlines indicate closure, as subgroups of the affine function vector spaces; of course, there is no requirement that any of the three overlined groups be real vector spaces (they are norm-complete subgroups). The two leftmost top vertical arrows are just induced by the affine representations; the right one is the inclusion, compatible with $\Sigma$ restricted to the image of $\operatorname{ker} \phi$. The two leftmost bottom vertical arrows are the obvious inclusions. The $\Sigma$ in the middle row is an abuse of notation; it represents the restriction of $\Sigma$ to $\overline{\operatorname{ker} \phi}$, the closed subgroup of $\phi^{\vdash}$, but the notation is already rather complex.

The middle row need not be exact at either end. For example, if $\operatorname{ker} \phi$ has dense image in $\phi^{\vdash}$ but one or both of $\operatorname{ker} \sigma$ or $\operatorname{ker} \tau$ does not have dense image in $\sigma^{\vdash}$ or respectively $\tau^{\vdash}$, then it is not left exact; if $\sigma(G) \cap \tau(H)$ is discrete, then the middle line is not right exact.

If $\operatorname{ker} \phi$ has dense image in $\phi^{\vdash}$, then $\sigma(G) \cap \tau(H)$ is a dense subgroup of $\mathbb{R}$ : we simply note that density of the image of $\operatorname{ker} \phi$ in $\phi^{\vdash}$, the latter being a closed and therefore a norm-complete subspace of $\operatorname{Aff} S(K,(u, v))$, entails that for every bounded linear functional that is not zero on $\phi^{\vdash}$, its restriction to a dense subgroup must be not zero and have dense range in the reals.

It also leads to a straightforward proof that if $\operatorname{ker} \sigma$ and $\operatorname{ker} \tau$ have dense images in $\sigma^{\vdash}$ and $\tau^{\vdash}$ respectively, and if $\sigma(G) \cap \tau(H)$ is a dense subgroup of $\mathbb{R}$, then $\operatorname{ker} \phi$ has dense image in $\phi^{\vdash}$. We have that

$$
\sigma^{\vdash} \times \tau^{\vdash}=\overline{\widehat{\operatorname{ker} \sigma}} \times \overline{\widehat{\operatorname{ker} \tau}} \subseteq \overline{\widehat{\operatorname{ker} \phi}} \subseteq \phi^{\vdash} .
$$

The left and right terms of these inclusions are vector spaces, and since $\sigma^{\vdash} \times \tau^{\vdash}$ is a closed codimension-two subspace of Aff $S(K,(u, v))$ and $\phi^{\vdash}$ is codimension one, it follows that $\sigma^{\vdash} \times \tau^{\vdash}$ is a codimension-one subspace of $\phi^{\vdash}$. (The proof does not stop here - we do not know that $\widehat{\operatorname{ker} \phi}$ is a real vector space.)

The map $\Sigma$ induces a map from 
to a subgroup of the reals. However, this subgroup of the reals includes the dense subgroup $\sigma(G) \cap \tau(H)$, and as $\widehat{\operatorname{ker} \phi}$ is a norm-complete abelian group, the image must be complete, and thus must be onto. In addition, since

$$
\operatorname{ker} \Sigma=\sigma^{\vdash} \times \tau^{\vdash}=\overline{\widehat{\operatorname{ker} \sigma}} \times \overline{\widehat{\operatorname{ker} \tau}},
$$

it follows that

$$
\operatorname{ker} \Sigma \cap \overline{\widehat{\operatorname{ker} \phi}}=\overline{\widehat{\operatorname{ker} \sigma}} \times \overline{\widehat{\operatorname{ker} \tau}}
$$

We thus have

$$
\operatorname{ker} \Sigma \subset \widehat{\widehat{\operatorname{ker} \phi}} \subseteq \phi^{\vdash}
$$

but $\Sigma$ induces the equality

$$
\overline{\widehat{\operatorname{ker} \phi}} /(\operatorname{ker} \Sigma \cap \overline{\widehat{\operatorname{ker} \phi}})=\phi^{\vdash} / \operatorname{ker} \Sigma .
$$

It follows immediately that $\overline{\operatorname{ker} \phi}=\phi^{\vdash}$.

Now we can show that if the closure of the images of $\operatorname{ker} \sigma$ and $\operatorname{ker} \tau$ are real vector spaces, and if $\operatorname{ker} \phi$ is order unit good, then $\sigma$ and $\tau$ are order unit good.

We wish to show

$$
\overline{\widehat{\operatorname{ker} \sigma}} \times \overline{\widehat{\operatorname{ker} \tau}}=\sigma^{\vdash} \times \tau^{\vdash},
$$

as from this it follows trivially that

$$
\overline{\widehat{\operatorname{ker} \sigma}}=\sigma^{\vdash} \text { and } \overline{\widehat{\operatorname{ker} \tau}}=\tau^{\vdash} \text {. }
$$

Since the left thing is a vector space, and a complete normed abelian group (hence a closed vector subspace of Aff $S(K,(u, v)))$, if equality does not hold, there exists a bounded linear functional $\alpha$ on $\operatorname{Aff} S(K,(u, v))$ that kills $\overline{\operatorname{ker} \sigma} \times \widehat{\widehat{\operatorname{ker} \tau}}$ but not $\sigma^{\vdash} \times \tau^{\vdash}$; in particular, $\alpha$ does not kill $\phi^{\vdash}$.

By composition with the affine representation, we "restrict" $\alpha$ to a real-valued bounded group homomorphism $\beta: G \oplus H \rightarrow \mathbb{R}$ (for a treatment of bounded group homomorphisms on dimension groups, see [Goodearl 1986]; their behaviour is just what you'd expect). Since $\alpha$ kills $\widehat{\operatorname{ker} \sigma} \times \overline{\operatorname{ker} \tau}$, it follows that $\beta$ kills $\operatorname{ker} \sigma \oplus \operatorname{ker} \tau$. We form the normed abelian group $\operatorname{ker} \phi /(\operatorname{ker} \sigma \oplus \operatorname{ker} \tau)$, which via $\sigma$, we know to be $\sigma(G) \cap \tau(H) \subset \mathbb{R}$. Thus $\beta$ induces a bounded real-valued group homomorphism on $\operatorname{ker} \phi /(\operatorname{ker} \sigma \oplus \operatorname{ker} \tau)$; call it $\bar{\beta}$. We thus have two bounded group homomorphisms on the quotient, $\bar{\beta}$ and $\bar{\sigma}$, but as the quotient is isomorphic (as a normed abelian group) to a subgroup of the reals, there must be a positive real number $\lambda$ such that $\bar{\beta}=\lambda \bar{\sigma}$.

This forces $\beta=\lambda \cdot \sigma \circ \pi_{G}$ (as bounded group homomorphisms on ker $\phi$ ). Since ker $\phi$ has dense image in its completion (!) which happens to be $\phi^{\vdash}$, we have that $\alpha \mid \phi^{\vdash}=\lambda \Sigma$. Thus $\alpha$ kills $\sigma^{\vdash} \times \tau^{\vdash}$, a contradiction.

To summarize, we have the following results. 
Proposition 4.5. Suppose $(G, u, \sigma)$ and $(H, v, \tau)$ are nearly divisible dimension groups with order unit and distinguished trace, and form $K=G \oplus H$, and the trace $\phi=\sigma \oplus \tau: K \rightarrow \mathbb{R}$.

(a) If $\phi$ is order unit good (with respect to either the usual or the strict ordering on $K)$, then $\sigma(G) \cap \tau(H)$ is a dense subgroup of the reals, and $\sigma \otimes 1_{\mathbb{Q}}$ and $\tau \otimes 1_{\mathbb{Q}}$ are order unit good as traces on $G \otimes \mathbb{Q}$ and $H \otimes \mathbb{Q}$ respectively.

(b) If the closure of the images of $\operatorname{ker} \sigma$ and $\operatorname{ker} \tau$ in $\sigma^{\vdash}$ and $\tau^{\vdash}$ respectively are real vector spaces, and if $\phi$ is order unit good, then both $\sigma$ and $\tau$ are order unit good.

(c) If $\sigma$ and $\tau$ are order unit good and $\sigma(G) \cap \tau(H)$ is dense in $\mathbb{R}$, then $\phi$ is order unit good.

We can also rephrase this as follows.

Proposition 4.6. Let $(G, u, \sigma)$ and $(H, v, \tau)$ be nearly divisible dimension groups with order units and normalized traces. Consider the following properties:

(1) $\sigma \oplus \tau$ is an order unit good trace on $G \oplus_{s} H$.

(2) $\sigma(G) \cap \tau(H)$ is a dense subgroup of $\mathbb{R}$.

(3) The closures of the images of $\operatorname{ker} \sigma$ and $\operatorname{ker} \tau$ in their respective affine spaces are real vector spaces.

(4) Both $\sigma$ and $\tau$ are order unit good traces.

Then the following implications hold: (1) implies (2); (1) and (3) jointly imply (4); (4) implies (3); (4) and (2) jointly imply (1).

Remark. The implications are invariant under the transformation $(x) \mapsto(5-x)$.

Examples exist (Example 4.8) where $G$ and $H$ are simple dimension groups that show that if $\phi$ is order unit good, then neither $\sigma$ nor $\tau$ (or exactly one of them) need be order unit good.

This method suggests what to do with multiple traces. Let $\left(G_{i}, u_{i}, \sigma_{i}\right)$, where $i=1,2, \ldots, n$, be approximately divisible dimension groups, each with order unit and (unnormalized) trace. Form $K=\bigoplus G_{i}$ with the strict ordering, and $\phi=\sigma_{1} \oplus \sigma_{2} \oplus \cdots \oplus \sigma_{n}: K \rightarrow \mathbb{R}$, and the map $T: K \rightarrow \mathbb{R}^{n}$ defined by $\phi\left(\left(g_{i}\right)\right)=$ $\sum \sigma_{i}\left(g_{i}\right)$ and $T\left(\left(g_{i}\right)\right)=\left(\sigma_{1}\left(g_{1}\right), \sigma_{2}\left(g_{2}\right), \ldots, \sigma_{n}\left(g_{n}\right)\right)$. Identify Aff $S\left(K,\left(\left(u_{i}\right)\right)\right)$ with the cartesian product Aff $S\left(G_{1}, u_{1}\right) \times \cdots \times \operatorname{Aff} S\left(G_{n}, u_{n}\right)$.

If $\left(g_{i}\right) \in \operatorname{ker} \phi$, then $\sigma_{n}\left(g_{n}\right)=-\sum_{i=1}^{n-1} \sigma_{i}\left(g_{i}\right)$, and we can interchange $n$ with any other integer less than $n$. In particular, $V:=\sigma_{n}^{-1}\left(\sigma_{n}\left(G_{n}\right) \cap\left(\sum_{i=1}^{n} \sigma_{i}\left(G_{i}\right)\right)\right)$ is independent of permutations and the range of $T$ on $\operatorname{ker} \phi$ is $T(V)$.

Extend $T$ to $\mathcal{T}$ : Aff $S\left(K,\left(u_{i}\right)\right) \rightarrow \mathbb{R}^{n}$ (sending $\left(j_{i}\right)$ to $\left(j_{i}\left(\sigma_{i}\right)\right)$ ). Restricted to $\phi^{\vdash}$, the range of $\mathcal{T}$ is exactly $(1,1,1, \ldots, 1)^{\perp}$, i.e., the entries add to zero. 
Now we can form the diagram analogous to the previous one.

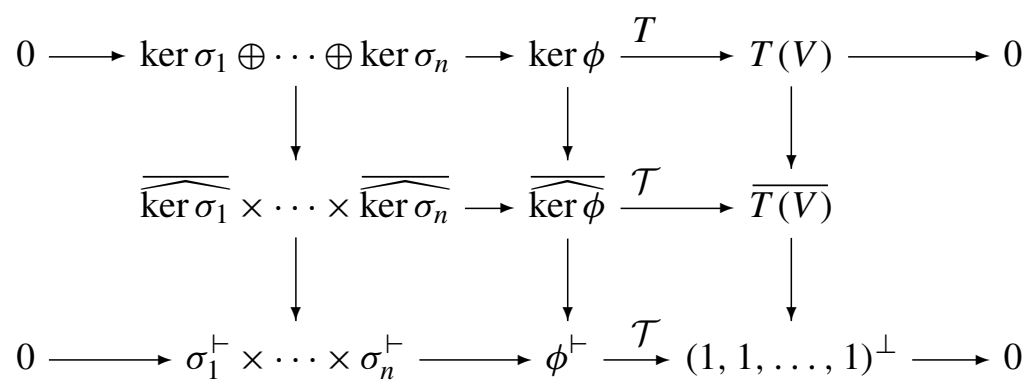

We quickly see that density of $T(V)$ (a subgroup of $\mathbb{R}^{n}$ contained in $(1, \ldots, 1)^{\perp}$ ) in $(1, \ldots, 1)^{\perp}$ is necessary for $\phi$ to be order unit good; that is, it is necessary in order for ker $\phi$ to have norm dense image in $\phi^{\vdash}$.

Suppose all the $\sigma_{i}$ are order unit good and $T(V)$ is dense in $(1, \ldots, 1)^{\perp}$. Then

$$
\overline{\overline{\operatorname{ker} \sigma_{1}}} \times \cdots \times \overline{\overline{\operatorname{ker} \sigma_{n}}}=\sigma_{1}^{\vdash} \times \cdots \times \sigma_{n}^{\vdash}
$$

is a closed subspace of $\phi^{\vdash}$, and the middle line yields that the codimension of $\overline{\overline{\operatorname{ker} \phi}}$ in Aff $S(K)$ is $n-(n-1)=1$, so being a closed subspace of the codimension-one space $\phi^{\vdash}, \widehat{\operatorname{ker} \phi}$ must equal it, and therefore $\phi$ is order unit good.

Suppose that $\phi$ is order unit good (hence we have density of $T(V)$ in $(1, \ldots, 1)^{\perp}$ ) and each $\widehat{\operatorname{ker} \sigma_{i}}$ is a vector space. To show that the $\sigma_{i}$ are all order unit good, it is sufficient to show that the $\operatorname{ker} \sigma_{i}$ have dense image in $\sigma^{\vdash}$, and it is easy to show that $\overline{\operatorname{ker} \sigma_{1}} \times \cdots \times \overline{\overline{\operatorname{ker} \sigma_{n}}}$ equals $\sigma_{1}^{\vdash} \times \cdots \times \sigma_{n}^{\vdash}$ is sufficient for this.

We note that the bounded real-valued group homomorphisms on $T(V)$, and of course on its closure, are linear combinations of the coordinate functions, which correspond to the $\sigma_{i}$, with lack of uniqueness arising from the relation that the sum of the coefficients is zero.

By assumption, each $\widehat{\operatorname{ker} \sigma_{i}}$ is a vector space (and closed in $\operatorname{Aff} S\left(G_{i}, u_{i}\right)$ ), whence the whole batch $L:=\overline{\operatorname{ker} \sigma_{1}} \times \cdots \times \overline{\operatorname{ker} \sigma_{n}}$ is a closed subspace of $M:=$ $\sigma_{1}^{\vdash} \times \cdots \times \sigma_{n}^{\vdash}$ (which is itself a closed codimension- $n$ subspace of Aff $S(K)$ ). If they are not equal, there exists a bounded linear functional $\alpha$ on $\operatorname{Aff} S\left(K,\left(u_{i}\right)\right)$ that kills $L$ but not $M$. This induces a bounded real-valued group homomorphism $\beta$ on $\operatorname{ker} \phi$ which kills $\operatorname{ker} \sigma_{1} \oplus \cdots \oplus \operatorname{ker} \sigma_{n}$. Hence it induces a bounded real-valued group homomorphism on the quotient, $T(V), B: T(V) \rightarrow \mathbb{R}$.

Each $\sigma_{i}$ induces $\Sigma_{i}$ on $T(V)$, and these are the coordinate functions. Hence there exist real $\lambda_{i}$ such that $B=\sum \lambda_{i} \Sigma_{i}$. Thus $\beta-\sum \lambda_{i} \sigma_{i}$ vanishes identically on $\operatorname{ker} \phi$, and by density, $\alpha=\sum \lambda_{i} \sigma_{i}$ (where $\sigma_{i}$ is now interpreted as the map $\left(j_{i}\right) \mapsto j_{i}\left(\sigma_{i}\right)$ on Aff $\left.S(K)\right)$. But this obviously kills $\sigma_{1}^{\vdash} \times \cdots \times \sigma_{n}^{\vdash}$, a contradiction. Hence each $\sigma_{i}$ is order unit good.

To summarize what happens with multiple traces, we have the following: 
Theorem 4.7. Let $\left(G_{i}, u_{i}, \sigma_{i}\right)$ be approximately divisible dimension groups with order unit $\left(u_{i}\right)$ and (unnormalized) trace $\left(\sigma_{i}\right)$. Form $K=\bigoplus G_{i}$ (with the strict ordering), and the trace $\phi=\bigoplus \sigma_{i}$ on $K$. Set $J=\sigma_{n}\left(G_{n}\right) \cap\left(\sum_{i \leq n-1} \sigma_{i}\left(G_{i}\right)\right)$, a subgroup of $\mathbb{R}$.

(a) If $\phi$ is order unit good, then $T\left(\sigma_{n}^{-1}(J)\right)$ is dense in $(1,1, \ldots, 1)^{\perp}$.

(b) If the closure of the image of $\operatorname{ker} \sigma_{i}$ in $\sigma_{i}^{\vdash}$ is a real vector space for all $i$, and if $\phi$ is order unit good, then all $\sigma_{i}$ are order unit good.

(c) If the image of $\operatorname{ker} \sigma_{i}$ is dense in $\sigma_{i}^{\vdash}$ for all $i$ (that is, each $\sigma_{i}$ is order unit good), and if $T\left(\sigma_{n}^{-1}(J)\right)$ is dense in $(1,1, \ldots, 1)^{\perp}$, then $\phi$ is order unit good.

The conditions for order unit goodness with $n$ direct summands are slightly different, in that they involve the density of a subgroup of $\mathbb{R}^{n-1}$ (identified with $\left.(1, \ldots, 1)^{\perp}\right)$, or simply that the closure of $T(V)$ is a vector space of dimension $n-1$ (in general, the closure need not be a vector space). To some extent, this explains some of the phenomena illustrated in the examples below, with direct sums of two not yielding an order unit good trace, but direct sums of three doing so. In fact, the argument in the example, $G_{n}=\mathbb{Z}+(\sqrt{3}+n \sqrt{2}) \mathbb{Z}$, essentially boils down to showing the closure of $T(V)$ is a two-dimensional vector space. But actually calculating with $T(V)$ seems awkward.

However, computation is feasible in special cases. Suppose $G_{1}=\mathbb{Z}+\sqrt{6} \mathbb{Z}$, $G_{2}=\mathbb{Z}+\sqrt{15} \mathbb{Z}$, and $G_{3}=\mathbb{Z}+\sqrt{10} \mathbb{Z}$. Then $T(V)$ is discrete, so $\sigma_{1} \oplus \sigma_{2} \oplus \sigma_{3}$ is not order unit good. However, if we add a fourth term, $G_{4}=\mathbb{Z}+(\sqrt{6}+\sqrt{15}+\sqrt{10}) \mathbb{Z}$, then with $\phi=\bigoplus_{i \leq 4} \sigma_{i}$,

$$
\begin{aligned}
\operatorname{ker} \phi=\{(a+b \sqrt{6}, c+b \sqrt{15}, d+b \sqrt{10}, & \\
& -(a+c+d)-b(\sqrt{6}+\sqrt{15}+\sqrt{10})) \mid a, b, c, d \in \mathbb{Z}\} .
\end{aligned}
$$

Let $v_{1}=(1,0,0,-1), v_{2}=(0,1,0,-1)$, and $v_{3}=(0,0,1,-1)$; then $\operatorname{ker} \phi$ is the $\mathbb{Z}$-span of

$$
\left\{v_{1}, v_{2}, v_{3}, \sqrt{6} v_{1}+\sqrt{15} v_{2}+\sqrt{10} v_{3}\right\} .
$$

The map from ker $\phi$ to $\mathbb{R}^{3}$ given by $v_{i} \mapsto e_{i}$ (standard basis elements) has range equal to the free abelian group on $\left\{e_{1}, e_{2}, e_{3}, \sqrt{6} e_{1}+\sqrt{15} e_{2}+\sqrt{10} e_{3}\right\}$. Since $\{1, \sqrt{6}, \sqrt{10}, \sqrt{15}\}$ is linearly independent over the rationals, this group is dense. It is trivial that $\left\{v_{i}\right\}$ is a real basis for $\phi^{\vdash}$, so $\phi$ is good. In this example, all the $\operatorname{ker} \sigma_{i}$ are trivial, so $T(V)$ is all of $\operatorname{ker} \phi$.

On the other hand, if we omit any one or two of the $G_{i}$, the resulting trace is not order unit good, since the resulting $T(V)$ will be discrete.

We can similarly construct $\left(G_{i}, \sigma_{i}\right)$ (the $G_{i}$ subgroups of the reals), $i=1, \ldots, n$, such that $\bigoplus_{i=1}^{n} \sigma_{i}$ is order unit good, but for no proper subset $J$ of $\{1,2, \ldots, n\}$ with $|J|>1$ is $\bigoplus_{i \in J} \sigma_{i}$ order unit good: Let $\left\{p_{i}\right\}_{i=1}^{n}$ be distinct primes; set 
$G_{i}=\mathbb{Z}+\sqrt{p_{i}} \mathbb{Z}$ for $1 \leq i \leq n-1$, and $G_{n}=\mathbb{Z}+\left(\sum_{i=1}^{n-1} \sqrt{p_{i}}\right) \mathbb{Z}$. The resulting $T(V)$ will be a critical group of rank $n$, so all subgroups of lesser rank are discrete.

Example 4.8. There exist simple dimension groups $(G, \sigma)$ and $(H, \tau)$ with traces such that $\phi=\sigma \oplus \tau$ is (order unit) good on the strict direct sum $K=G \oplus H$, but $\sigma$ is not good as a trace on $G$ (and in one case, $\tau$ is good, in another case, it is not).

Proof. For simple dimension groups (as $G, H$, and $K$ are), order unit goodness is equivalent to goodness. Begin with three subgroups of the reals,

$$
\begin{aligned}
& G_{1}=\mathbb{Z}+\sqrt{3} \mathbb{Z}+\sqrt{5} \mathbb{Z}, \\
& G_{2}=\mathbb{Z}+\sqrt{2} \mathbb{Z}+\sqrt{5} \mathbb{Z}, \\
& G_{3}=\mathbb{Z}+(\sqrt{3}+\sqrt{2}) \mathbb{Z} .
\end{aligned}
$$

Let $\tau_{i}$ denote the respective identifications of $G_{i}$ with subgroups of the reals; these are traces on each of these three totally ordered dimension groups. Each $\tau_{i}$ is the unique (up to scalar multiple) trace, and thus is good. Now form $L=G_{1} \oplus G_{2}$ with the strict order; since both subgroups contain $\mathbb{Z}+\sqrt{5} \mathbb{Z}$, which is dense, it follows from the criterion above that $\tau_{1} \oplus \tau_{2}$ is a good trace thereon. Next, form $K=L \oplus G_{3}$, with the strict order; since the value group of $\tau_{1} \oplus \tau_{2}(L)$ includes $\mathbb{Z}+(\sqrt{3}+\sqrt{2}) \mathbb{Z}$ and the latter is dense, we can apply the criterion again, and so deduce that $\tau_{1} \oplus \tau_{2} \oplus \tau_{3}$ is good, as a trace on $K$.

However, we can obtain $K$ by proceeding in a different order. Set $G=G_{1} \oplus G_{3}$ with the strict order. Either by direct examination or by the necessity of the density condition, $\tau_{1} \oplus \tau_{3}$ is not good - note in particular, that the intersection of the value groups is just $\mathbb{Z}$. Let $H=G_{2}$; then the obvious permutation-order isomorphism which takes $K$ to $G \oplus H$ takes $\tau_{1} \oplus \tau_{2} \oplus \tau_{3}$ to $\tau_{1} \oplus \tau_{3} \oplus \tau_{2}$, hence the latter is good. But with $\sigma=\tau_{1} \oplus \tau_{3}$ and $\tau=\tau_{2}$, we have that $\sigma$ is not good, whereas $\sigma \oplus \tau$ (and $\tau$ ) is good.

To obtain an example wherein neither $\sigma$ nor $\tau$ is good, let $G_{4}$ be another copy of $G_{3}$, set $G=G_{1} \oplus G_{3}$ and $H=G_{2} \oplus G_{4}$ (with the strict orderings of course); $\tau=$ $\tau_{2} \oplus \tau_{4}$ is not good for the same reason as $\sigma=\tau_{1} \oplus \tau_{3}$, but their direct sum is good.

\section{Good sets of traces}

As in [BeH 2014], a compact convex subset $Y$ of $S(G, u)$ is order unit good with respect to $(G, u)$ if given $b \in G^{+} \backslash\{0\}$ ( $b$ is an order unit of $\left.G\right)$ and $a \in G$ such that $0 \ll \hat{a}|K \ll \hat{b}| K$, there exists $a^{\prime} \in G$ such that $\hat{a}\left|K=\hat{a}^{\prime}\right| K$ and $0 \leq a^{\prime} \leq b$. When $Y$ is a face (it need not be; e.g., for any singleton subset of $S(G, u),\{\tau\}$ is good if and only if the trace $\tau$ is good as defined for individual traces), $Y$ is order unit good if and only if ker $Y:=\bigcap_{\tau \in K} \operatorname{ker} \tau$ has dense range in $Y^{\perp}=\{h \in \operatorname{Aff} S(G, u)|h| K \equiv 0\}$. When $G$ is simple, this was defined as good in [BeH 2014]. When $G=\operatorname{Aff} K$ (where $K$ is a Choquet simplex), equipped with the strict ordering, goodness of 
subsets of $K$ is an interesting geometric property. In Appendix B, we show that at least when $K$ is finite-dimensional, the good subsets of $K$ are as conjectured in [BeH 2014, Section 7, Conjecture].

There is a problem in defining what a good subset $Y$ should be in the nonsimple case. It should be consistent with what has been defined in the simple case (where good is the same as order unit good), and the singleton case (whence came the original definition of good); additionally, it would be desirable that if $Y=S(G, u)$, then $Y$ should be good whenever $G$ is a dimension group such that $\operatorname{Inf} G=\{0\}$.

We give a definition of good in more complicated situations, including for a set of traces; this extends some of the definitions in [BeH 2014]. For any partially ordered abelian group $H$ and $h \in H^{+}$, recall the definition of the interval generated by $h$, denoted $[0, h]$ (possibly with a subscript $H$ if necessary to avoid ambiguity about the choice of group), to be $\{j \in H \mid 0 \leq j \leq h\}$. Let $(G, u)$ be a dimension group (at this stage, we really only require that it be a partially ordered unperforated group) with order unit. Let $L$ be a subgroup of $G$; we say $L$ is a good subgroup of $G$ if

(i) $L$ is convex (that is, if $a \leq c \leq b$ with $a, b \in L$ and $c \in G$, then $c \in L$ ), and $G / L$ is unperforated;

(ii) using the quotient map $\pi: G \rightarrow G / L$, the latter equipped with the quotient ordering, for every $b \in G^{+}$, we have $\pi([0, b])=[0, \pi(b)]$.

Convexity is required in order that the quotient positive cone be proper, that is, the only positive and negative elements are zero. Unperforation is often redundant; it may always be (in the presence of (ii); see the discussion concerning refinability in [BeH 2014]). The second property says that for all $b \in G^{+}$, and for all $a \in G$ such that $0 \leq a+L \leq b+L$ (or equivalently, $(a+L) \cap G^{+}$and $(b-a+L) \cap G^{+}$ are both nonempty), there exists $a^{\prime} \in G$ such that $a-a^{\prime} \in L$ and $0 \leq a^{\prime} \leq b$. This is a strong lifting property.

For example, if $\tau$ is a trace, set $L=\operatorname{ker} \tau$; this is convex, and is a good subgroup of $G$ if and only if $\tau$ is good (as a trace); in this case, $G / L$ is naturally isomorphic to a subgroup of the reals, so unperforation is automatic.

For a subset $U$ of $S(G, u)$ define $\operatorname{ker} U=\bigcap_{\sigma \in U} \operatorname{ker} \sigma$; for a subset $W$ of $G$, define $Z(W)=\{\sigma \in S(G, u) \mid \sigma(w)=0\}$. For good sets, we may as well assume that $Y=Z(\operatorname{ker} Y)$ at the outset; in other words, $\sigma \in Y$ if and only if $\sigma(\operatorname{ker} K)=0$, since in any reasonable definition for good or order unit good, the candidate set will satisfy $Y=Z(\operatorname{ker} Y)$. As explained in [BeH 2014], these form the collection of closed sets with respect to a Zariski-like topology, and also extend the definition of facial topology (relative to $G$ ) defined on $\partial_{e} S(G, u)$, to $S(G, u)$. If $Y \subset S(G, u)$, set $\widetilde{Y}=$ $Z(\operatorname{ker} Y)=\{\sigma \in S(G, u) \mid \sigma(\operatorname{ker} Y)=\{0\}\}$; this is a closure operation, corresponding to the facial topology and analogous to the Zariski topology from algebraic geometry. In many cases, we just assume $Y=\widetilde{Y}$ already, since $\operatorname{ker} Y=\operatorname{ker} \widetilde{Y}$. 
We say $Y$ is a good subset of $S(G, u)$ with respect to $(G, u)$ if $Y=\widetilde{Y}$ and $\operatorname{ker} Y$ is a good subgroup of $G$. If $Y=\{\tau\}$, and $\tau$ is merely an order unit good trace, then ker $\tau$ has dense image in $\tau^{\vdash}$, and this implies $Y=\widetilde{Y}$.

If $L$ is a subgroup of $G$, then we may form

$$
Y \equiv Z(L)=\{\sigma \in S(G, u) \mid \sigma(L)=\{0\}\} .
$$

Then $Y$ satisfies $\widetilde{Y}=Y$. However, it can happen that $L$ is a good subgroup of $G$, but $Z(L)$ is not a good subset of $S(G, u)$ with respect to $G$.

For example, let $\left(H,\left[\chi_{X}\right]\right)$ be the ordered Čech cohomology group of any noncyclic primitive subshift of finite type. It is known not to be a dimension group, but is unperforated and has numerous other properties (see [Boyle and Handelman 1996] as well as unpublished results of Boyle and the author). There exists a dimension group $(G, u)$ such that $H \cong G / \operatorname{Inf} G$ with the quotient ordering. Set $Y=S(G, u)$, so that $\operatorname{ker} Y=\operatorname{Inf} G$. Since the quotient $H=G / \operatorname{Inf} G$ is not a dimension group, it follows from results below that property (ii) fails. However, $L=\{0\}$ is clearly a good subgroup of $G$, and $Z(L)=Y$, but $\operatorname{ker} Y=\operatorname{Inf} G$. So $Y$ is not a good subset of $S(G, u)$.

In the definition of a good subgroup, it may be that the relatively strong condition that $G / L$ is unperforated can be replaced by the much weaker $G / L$ is torsionfree, in the presence of (ii), the lifting property. This is the case in the situation described in [BeH 2014, Proposition 7.6], dealing with simple dimension groups and $L=\operatorname{ker} Y$. There are criteria for the quotient $G / L$ to be unperforated $[\mathrm{BeH}$ 2014, Lemma B1], but these are not always easy to verify.

The following is implicit in [BeH 2014, Proposition 7.6].

Lemma 5.1. Suppose $(G, u)$ is a dimension group and $L$ is a convex subgroup of $G$ satisfying (ii). Then $G / L$ with the quotient ordering is an interpolation group, and its trace space is canonically affinely homeomorphic to $L^{\vdash}$. The latter is a Choquet simplex.

Proof. If $0 \leq a+L \leq(b+L)+(c+L)$ in $G / L$, first lift $b$ and $c$ separately to positive elements of $G$; it doesn't hurt to relabel them $b$ and $c$. Applying (ii) to $0 \leq a+L \leq(b+c)+L$, we can find $a^{\prime} \in[0, b+c]$ such that $a-a^{\prime} \in L$. Hence $0 \leq a^{\prime} \leq b+c$; by interpolation in $G$, we may find $a_{1} \in[0, b]$ and $a_{2} \in[0, c]$ such that $a^{\prime}=a_{1}+a_{2}$. Then $a+L=a^{\prime}+L=\left(a_{1}+L\right)+\left(a_{2}+L\right)$ and $a_{i}+L$ are positive elements of $G / L$, and each is less than $b+L, c+L$ respectively. Thus $G / L$ satisfies Riesz decomposition. The rest is standard.

We may consider the simplest definition possible for goodness of a set; that is, $Y$ is better (a facetious, but not inapt, name) for $(G, u)$ if (i) $Y=Z(\operatorname{ker} Y)$ and (ii) whenever $a \in G, b \in G^{+}$and $0 \leq \hat{a}|Y \leq \hat{b}| Y$, there exists $a^{\prime} \in G^{+}$such that $\hat{a}^{\prime}|Y=\hat{a}| Y$ and $a^{\prime} \leq b$. This turns out to be much too restrictive (although it is an 
interesting property); for example, if $Y=S(G, u)$, then $Y$ is better implies $G / \operatorname{Inf} G$ (with the quotient ordering; this need not be a dimension group) is archimedean, which hardly ever occurs; and if $G$ is simple, this is generally stronger than order unit good. If $Y$ is a singleton, then better agrees with the original definition of good.

Lemma 5.2. Let $(G, u)$ be a dimension group with order unit $u$. If $Y \subseteq S(G, u)$ is good with respect to $(G, u)$, then $G / \operatorname{ker} Y$ is a dimension group, with trace space canonically affinely homeomorphic to $Y$.

Proof. As good implies order unit good, ker $Y$ has dense image in $Y^{\vdash}$, and thus its closure is a vector space, so that by [BeH 2014, Corollary B2], $G / \operatorname{ker} Y$ is unperforated. Now suppose $0 \leq a+\operatorname{ker} Y \leq(b+\operatorname{ker} Y)+\left(b^{\prime}+\operatorname{ker} Y\right)$, where the latter two terms are nonnegative. Hence we may assume $b, b^{\prime} \geq 0$, and thus $0 \leq a+\operatorname{ker} Y \leq\left(b+b^{\prime}\right)+\operatorname{ker} Y$ implies there exists $a^{\prime} \in G^{+}$such that $a^{\prime}+\operatorname{ker} Y=a+\operatorname{ker} Y$ and $a^{\prime} \leq b+b^{\prime}$. Riesz interpolation in $G$ yields a decomposition $a^{\prime}=a_{1}+a_{2}$, where $0 \leq a_{1}, a_{2}$ and $a_{1} \leq b$ and $a_{2} \leq b^{\prime}$. Hence $a+\operatorname{ker} Y=a^{\prime}+\operatorname{ker} Y=\left(a_{1}+\operatorname{ker} Y\right)+\left(a_{2}+\operatorname{ker} Y\right)$, and $a_{1}+\operatorname{ker} Y \leq b+\operatorname{ker} Y$, and $a_{2}+\operatorname{ker} Y \leq b^{\prime}+\operatorname{ker} Y$. Thus $G / \operatorname{ker} Y$ satisfies interpolation.

Any trace $\tau$ of $G / \operatorname{ker} Y$, normalized at $u+\operatorname{ker} Y$, induces a trace $\tilde{\tau}$ of $(G, u)$ by composing with the quotient map. Conversely, if $\sigma$ is a trace that kills $\operatorname{ker} Y$, then from the definition, $\sigma \in Y$. Hence the map $S(G / \operatorname{ker} Y, u+\operatorname{ker} Y) \rightarrow S(G, u)$ is oneto-one and onto, and it is easy to see that it is an affine homeomorphism to Aff $Y$.

Lemma 5.3. If $Y$ is a good subset of $S(G, u)$, then $(I, w)$ is an order ideal of $G$ with its own order unit, and for all $\sigma \in Y$, we have $\sigma \mid I \not \equiv 0$, then the map

$$
I /(I \cap \operatorname{ker} Y) \rightarrow G / \operatorname{ker} Y
$$

is an order isomorphism.

Proof. First we show $I /(\operatorname{ker} Y \cap I)$ is unperforated, by showing the image of $I$ is an order ideal in $G / \operatorname{ker} Y$ (which is unperforated, by the preceding). Select $0 \leq a+\operatorname{ker} Y \leq b+\operatorname{ker} Y$, where $b \in I$; we can write $b=b_{1}-b_{2}$, where $b_{i} \in I^{+}$, and thus $0 \leq a+\operatorname{ker} Y \leq b_{1}+\operatorname{ker} Y$, and now $b_{1} \in I^{+}$. There thus exists $a^{\prime} \in\left[0, b_{1}\right]$ such that $a-a_{1} \in \operatorname{ker} Y$. As $0 \leq a^{\prime} \leq b_{1}$ and $b_{1} \in I$, it follows that $a_{1} \in I^{+}$, so that $a_{1}+\operatorname{ker} Y$ is in the image of $I$; the latter is thus a convex subgroup of $G / \operatorname{ker} Y$. Directedness of the image is trivial, so $I /(I \cap \operatorname{ker} Y)$ is an order ideal in $G / \operatorname{ker} Y$.

Any order ideal in an unperforated partially ordered group is itself unperforated, so $I /(\operatorname{ker} Y \cap I)$ is unperforated.

If $\sigma \in Y$ and $\sigma(w)=0$, then $\sigma(I)=0$, contradicting the property of $Y$; hence $\hat{w} \mid Y \gg \delta$ for some $\delta>0$. Since $G / \operatorname{ker} Y$ is unperforated and its trace space is canonically identified with $Y$, it follows that $w+\operatorname{ker} Y$ is an order unit for $G / \operatorname{ker} Y$. Hence the order ideal generated by $w+\operatorname{ker} Y$ is all of $G / \operatorname{ker} Y$. Hence the image of $I$ in $G / \operatorname{ker} Y$ is onto. 
So far, the map $I /(I \cap \operatorname{ker} Y) \rightarrow G / \operatorname{ker} Y$ is one-to-one (by construction), orderpreserving (by definition), and now we know that it is onto. To show it is an order-isomorphism, it suffices to show that the image of $I^{+}$is all of the positive cone.

Select $b \in G^{+}$. Then $\hat{b} \mid Y \ll m$ for some integer $m$, so there exists an integer $N$ such that $\hat{b} \ll N \hat{w}$, and thus $0 \leq b+\operatorname{ker} Y \leq N w+\operatorname{ker} Y$ (the latter by unperforation, again). By goodness, there exists $a \in[0, N w]$ such that $a-b \in \operatorname{ker} Y ; 0 \leq a \leq N w$ implies $a \in I^{+}$, and it maps to $b+\operatorname{ker} Y$.

The latter property is the analogue of $\tau(I)=\tau(G)$ for a single good trace $\tau$ of $G$. If we weaken the hypotheses to "ker $Y$ does not contain $I$ ", then the result is unclear. We have similar problems with the following characterization when some points of $Y$ are not faithful.

Lemma 5.4. Let $(I, w)$ be an order ideal of $G$ with its own order unit, and suppose that every point of $Y$ does not kill $I$. Then the map $\phi_{I}: Y \rightarrow S(I, w)$, given by $\sigma \mapsto \sigma / \sigma(w) \mid I$, is continuous. If $Y$ is good with respect to $(G, u)$, then $\phi_{I}(Y)$ is good with respect to $(I, w)$.

Proof. The restriction map on traces sends every point to a nonzero trace of $I$, and thus the map is continuous (as $Y$ is compact, $\inf _{\sigma \in Y} \sigma(w)>0$ ). Suppose $\rho$ is a normalized trace on $(I, w)$ such that $\rho \mid(I \cap \operatorname{ker} \rho)$ is identically zero. Then $\rho$ induces a trace on $I /(I \cap \operatorname{ker} Y)$, hence is a trace on $G / \operatorname{ker} Y$, and therefore $\rho$ is the restriction of a trace from $G$, necessarily killing $Y$. If $r$ is the lifted trace, we must have $r \in Y$, and thus $\rho \in \phi_{I}(Y)$. In particular, relative to $(I, w)$, we have $\phi_{I}(Y)=Z\left(\operatorname{ker} \phi_{I}(Y)\right)$, and it follows immediately that $\phi_{I}(Y)$ is good with respect to $(I, w)$.

The condition on $Y$ in the next result, that every point be faithful, is rather strong, but makes things easier to deal with. The much weaker faithfulness condition (ker $Y \cap G^{+}=\{0\}$ ) is innocuous, as we can factor out the maximal order ideal contained in $\operatorname{ker} Y$.

Lemma 5.5. Let $(G, u)$ be a dimension group, and $Y$ a subset of $S(G, u)$ for normalized traces $\sigma$. Then $\sigma \mid \operatorname{ker} Y \equiv 0$ if and only if $\sigma \in Y$ and $\operatorname{ker} Y \cap G^{+}=\{0\}$.

(a) The trace space of the quotient $G / \operatorname{ker} Y$ is canonically affinely homeomorphic to $Y$.

(b) If $G / \operatorname{ker} Y$ is unperforated and $Y$ satisfies the additional condition that every element of $Y$ is faithful, then $G / \operatorname{ker} Y$ is simple.

Proof. Let $\phi$ be a normalized trace of $(G / \operatorname{ker} Y, u+\operatorname{ker} Y)$, and let $\pi: G \rightarrow G / \operatorname{ker} Y$ be the quotient map. Then $\sigma^{\prime}:=\sigma \circ \pi$ is a normalized trace of $(G, u)$ satisfying $\sigma(\operatorname{ker} Y)=0$, so $\sigma \in Y$. Thus the map $S(G / \operatorname{ker} Y, u+\operatorname{ker} Y) \rightarrow S(G, u)$ given by $\sigma \mapsto \sigma \circ \pi$ has image in $Y$, and is clearly onto $Y$.

(a) The map is obviously one-to-one, affine, and continuous, with continuous inverse $Y \rightarrow S(G / \operatorname{ker} Y, u+\operatorname{ker} Y)$, and so is an affine homeomorphism. 
(b) Suppose $a+\operatorname{ker} Y$ is nonzero and $a+\operatorname{ker} Y \geq 0+\operatorname{ker} Y$; there thus exists $a^{\prime} \geq 0$ such that $a^{\prime}-a \in \operatorname{ker} Y$ (from the definition of the ordering on the quotient group). If $a+\operatorname{ker} Y$ is not an order unit, then there exists a normalized trace $\sigma$ on $G / \operatorname{ker} Y$ such that $\sigma(a+\operatorname{ker} Y)=0$ (otherwise, $\hat{a} \mid Y$ is strictly positive, and as $G / \operatorname{ker} Y$ is unperforated, this would imply $a+\operatorname{ker} Y$ is an order unit in $G / \operatorname{ker} Y$ ). Then $\sigma^{\prime}=\sigma \circ \pi$ belongs to $Y$ and $\sigma^{\prime}\left(a^{\prime}\right)=0$, contradicting $\operatorname{ker} \sigma^{\prime} \cap G^{+}=\{0\}$.

Hence every nonzero element of $G / \operatorname{ker} Y$ is an order unit.

If in part (b), we drop the unperforated hypothesis, then we can still say something. From $a+\operatorname{ker} Y \geq 0+\operatorname{ker} Y$, we have $0 \leq \hat{a} \mid Y$; if for all positive integers $m$, we have that $m a+\operatorname{ker} Y$ is not an order unit in $G / \operatorname{ker} Y$, then there must exist a trace $\phi$ on $G / \operatorname{ker} Y$ such that $\phi\left(a^{\prime}\right)=0$. As in the argument above, this leads to a contradiction. So in the perforated case, we obtain that there exists $m>0$ such that $m(a+\operatorname{ker} Y)$ is an order unit. If we define simple to mean no proper order ideals, then the quotient group is simple. (We normally deal with unperforated order groups, wherein the lack of order ideals is equivalent to every nonzero nonnegative element being an order unit.)

The following is a variant of [BeH 2014, Lemma 7.1].

Lemma 5.6. Let $(G, u)$ be an approximately divisible dimension group, and let $L$ be a convex subgroup. If $G / L$ is unperforated, then order units lift. (That is, given a such that $a+L$ is an order unit of $G / L$, there exists an order unit $v$ of $G$ such that $a-v \in L$.)

Proof. The traces of $G / L$ are the traces of $G$ that kill $L, Z:=Z(L) \subset S(G, u)$. As $a+L$ is an order unit, $\hat{a} \mid L \gg \delta$ for some $\delta>0$. As $G$ is approximately divisible, there exists $w \in G$ such that $\delta / 3<\hat{w}<\delta / 2$. Then $(\hat{a}-\hat{w}) \mid Z \gg \delta / 2$; as $G / L$ is unperforated, $a-w+L$ is in $(G / L)^{+}$. From the definition of quotient ordering, there exists $c \in G^{+}$such that $c+L=a-w+L$. Set $v=c+w$. Then $v+L=a-w+w+L=a+L$; since $v \geq w$ and $w$ is an order unit, it follows that $v$ is an order unit.

If we drop approximate divisibility, we obtain that for all order units $a+L$ of $G / L$, there exists an integer $N$ such that for all $n \geq N$, there exist order units $v_{n}$ of $G$ such that $v_{n}-n a \in L$. (Instead of using a small order unit $w$, we take $u$ or any other order unit we can find.)

The following gives a general result (without assuming $G / \operatorname{ker} Y$ is unperforated, but instead, that $Y$ is a face) about lifting order units.

Lemma 5.7. Suppose $Y=Z(\operatorname{ker} Y)$ is a face of $S(G, u)$ such that the image of $\operatorname{ker} Y$ is dense in $Y^{\perp}$. Let $a \in G$ satisfy $a+\operatorname{ker} Y \geq 0$ and $\hat{a} \mid Y \gg \delta$ for some $\delta>0$. Then there exists $a^{\prime} \in G^{++}$such that $a^{\prime}+\operatorname{ker} Y=a+\operatorname{ker} Y$. 
Proof. From the quotient ordering, there exists $c \in G^{+}$such that $c-a \in \operatorname{ker} Y$. Let $F=\{\tau \in S(G, u) \mid \tau(c)=0\}$; because $c \in G^{+}, F$ is a face, and is obviously closed. Since $\hat{c}|Y=\hat{a}| Y$, we must have $F \cap Y=\varnothing$. There thus exists $h \in \operatorname{Aff} S(G, u)^{+}$ such that $h \mid Y \equiv 0$ and $h \mid F \equiv 1$.

As $h \in Y^{\perp}$, there exist $g_{n} \in \operatorname{ker} Y$ such that $\hat{g}_{n} \rightarrow h$ uniformly. Hence $\widehat{g_{n}+c} \rightarrow$ $h+\hat{c}$ uniformly. The latter however is strictly positive (since $\hat{c} \geq 0$ and $\hat{c}^{-1}(0)=F$ ). Hence there exists $n$ such that $\widehat{g_{n}+c}$ is strictly positive; as $G$ is unperforated, $a^{\prime}:=g_{n}+c$ is an order unit of $G$. Its image modulo $\operatorname{ker} Y$ is $c+\operatorname{ker} Y=a+\operatorname{ker} Y$.

Proposition 5.8. Suppose that $(G, u)$ is a nearly divisible dimension group, and $Y=Z(\operatorname{ker} Y)$ is a subset of $S(G, u)$ such that for all $\sigma \in Y$, $\operatorname{ker} \sigma \cap G^{+}=\{0\}$. Suppose that either $Y$ is a face or $G / \operatorname{ker} Y$ is unperforated. Then $Y$ is good (with respect to $(G, u))$ if and only if

(a) $\phi_{I}(Y)$ is order unit good for all order ideals I having their own order unit,

(b) for every nonzero order ideal $I$, we have $I+\operatorname{ker} Y=G+\operatorname{ker} Y$.

Remark. Condition (b) is just a restatement of the map $I /(I \cap \operatorname{ker} Y) \rightarrow G / \operatorname{ker} Y$ being onto. It does not require the stronger property, that it be an order isomorphism.

Proof. Sufficiency of the conditions: Suppose $b \in G^{+}$and $a \in G$ and in addition, $0 \leq a+\operatorname{ker} Y \leq b+\operatorname{ker} Y$. Let $I \equiv I(b)$ be the order ideal generated by $b$; that is, $I(b)=\{g \in G \mid \exists N \in \mathbb{N}$ such that $-N b \leq g \leq N b\}$. By (b), there exists $a_{1} \in I(b)$ such that $a_{1}+\operatorname{ker} Y=a+\operatorname{ker} Y$. Since $I /(I \cap \operatorname{ker} Y)$ is simple, $0 \leq a_{1}+\operatorname{ker} Y \leq b+\operatorname{ker} Y$ entails either $a_{1}+\operatorname{ker} Y=0+\operatorname{ker} Y$ or $a_{1}+\operatorname{ker} Y$ is an order unit. In the former case, set $a^{\prime}=0$.

Otherwise, if $Y$ is a face, there exists $a_{2} \in I^{++}$such that $a_{2}+\operatorname{ker} Y=a_{1}+\operatorname{ker} Y$. Similarly, either $b+\operatorname{ker} Y=a_{1}+\operatorname{ker} Y$ (in which case, we take $a^{\prime}=b$ ) or the difference $b+\operatorname{ker} Y-\left(a_{2}+\operatorname{ker} Y\right)$ is an order unit in $I /(\operatorname{ker} Y \cap I)$.

If $G / \operatorname{ker} Y$ is unperforated, then $I /(I \cap \operatorname{ker} Y)$ is unperforated (this follows from $I$ being an order ideal in $G$ ), and applying Lemma 5.6(b) to $\phi_{I}(Y)$, is simple with trace space canonically $\phi_{I}(Y)$. This means that the order-preserving one-to-one and onto map $I /(I \cap \operatorname{ker} Y) \rightarrow G / \operatorname{ker} Y$ induces an affine homeomorphism on their respective trace spaces; since the images in their affine function representations are the same, that of $I /(I \cap \operatorname{ker} Y)$ has dense range, and being simple (and $\phi_{I}(Y)$ being a simplex), the latter is a simple dimension group. A one-to-one order-preserving group homomorphism between simple dimension groups which induces an affine homeomorphism on the trace spaces is necessarily an order isomorphism.

Thus in either case, we have $0 \ll \hat{a}|Y \ll \hat{b}| Y$; now order unit goodness of $(I(b), b)$ yields $a^{\prime} \in I^{+}$such that $a^{\prime} \leq b$.

Necessity of the conditions follows from the preceding results. 
Now we briefly examine examples in $R_{P}$. When $R$ is a partially ordered commutative unperforated ring with 1 as an order unit, every closed face of $S(R, 1)$ is uniquely determined by its extreme points and these form a compact subset of $X=\partial_{e} S(R, 1)$ (and conversely, every closed subset of $X$ yields a closed face in this way). Thus, as a preliminary question, we can ask when the closed face obtained from the closed subset $Y$ of $X$ is good (for $R$ ) or order unit good. We say $Y$ generates an (order unit) good face when this occurs.

It is easy to see that $Y$ generates an order unit good face for $R$ if and only if for all pure traces $\sigma \notin Y$, we have $\sigma \mid \operatorname{ker} Y$ is not identically zero (we define $\operatorname{ker} Y=\bigcap_{\tau \in Y} \operatorname{ker} \tau$, as usual).

To verify this, if $Y$ generates an order unit good face for $R$, then $\operatorname{ker} Y$ has dense image in Ann $Y:=\{f \in C(X, \mathbb{R})|f| Y \equiv 0\}$. There exists $f \in$ Ann $Y$ such that $f(\sigma) \neq 0$, and there exist $a_{n} \in \operatorname{ker} Y$ such that $\hat{a}_{n} \rightarrow f$ uniformly, so there exists $a \in\left\{a_{n}\right\}$ such that $0 \neq \hat{a}(\sigma)=\sigma(a)$, hence $\sigma \mid \operatorname{ker} Y$ is not identically zero.

Conversely, suppose $\sigma(\operatorname{ker} Y) \neq\{0\}$ for every $\sigma \in X \backslash Y$. It is trivial that $\operatorname{ker} Y$ is an ideal of $R$ (not generally an order ideal), so its closure in $C(X, \mathbb{R})$ is a closed ideal thereof, hence of the form Ann $Z$ for some closed $Z \subset X$. Obviously $Y \subset X$, but if $\sigma \in Z \backslash Y$, there exists $a \in \operatorname{ker} Y$ such that $\sigma(a) \neq 0$, so that $\hat{a} \notin \operatorname{Ann} Z$, a contradiction. Hence $Z=Y$, so $\operatorname{ker} Y$ has dense image in Ann $Z$, and thus $Y$ is order unit good for $R$.

Lemma 5.9. Let $R$ be a partially ordered unperforated approximately divisible commutative ring, and let $Y$ be a compact subset of the set of faithful pure traces. Let $(I, v)$ be a nonzero order ideal with its own order unit.

(a) The set $Y$ maps by normalized restriction to a compact set of pure faithful traces on $(I, v), Y_{I}$.

(b) If the closed face generated by $Y$ is order unit good for $R$, then the closed face of $S(I, w)$ generated by $Y_{I}$ is order unit good for $(I, v)$.

Goodness for $R_{P}$ (several variables) of sets corresponding to faces (that is, closed subsets of the pure trace space) is dependent on the coefficients. For example, as we will see below, if $V$ is the variety given by $f=(x-3)^{2}+(y-3)^{2}-1$, the circle of radius one centred at $(3,3)$ and $P=c_{0}+c_{1} x+c_{2} y$, then $V$ (or its corresponding face in $S\left(R_{P}, 1\right)$ is order unit good, but not good, no matter what the choice of (positive) integers $c_{0}, c_{1}, c_{2}$. On the other hand, if $P_{1}=P \cdot Q$ where $Q=c+x f+y g+x y h$, where $f$ is a polynomial in $x$ with no negative coefficients such that $(x-3)^{2}+8$ divides some power of $c+x f$ (such exist!), $g$ is a polynomial in $y$ such that $(y-3)^{2}+8$ divides some power of $c+y g$, and $h$ is a polynomial in $x y^{-1}$ such that $\left(1+X^{2}\right)$ divides some power of $h(X)$, then $V$ is a good subset for $R_{P_{1}}$ (the conditions on the coefficients of monomials appearing in the faces of the Newton 
polytope described by the divisibility condition are necessary and sufficient for Proposition 5.8(b) to apply; however they are also extremely complicated).

Now we specialize to $R=R_{P}$ or $R_{P} \otimes \mathbb{Q}$, and to avoid severe complications, also assume that the compact $Y$ consists of pure faithful traces (that is, $Y$ is a compact subset of the positive orthant, $\left(\mathbb{R}^{d}\right)^{++}$, after identifying the pure faithful traces with points of the orthant). Then $\operatorname{ker} Y=\left\{f / P^{k} \in R_{P}|f| Y \equiv 0\right\}$. Recall that for $f \in$ $\mathbb{Z}\left[x_{1}, \ldots, x_{d}\right], f / P^{k} \in R_{P}$ means that there exists $l$ such that $\log f P^{l} \subseteq \log P^{k+l}$; we can well assume $\log f \subseteq \log P^{k}$.

Hence $Y$ is order unit good for $R$ if and only if whenever $\sigma$ is a pure trace not in $Y$, we have $\sigma \mid \operatorname{ker} Y \neq 0$. If we restrict $\sigma$ to the faithful pure traces, then we deduce a necessary condition: if $Y \subset\left(\mathbb{R}^{d}\right)^{++}$is compact, then $Y$ is order unit good for $R_{P}$ implies

$$
\left.Z I(Y) \cap\left(\mathbb{R}^{d}\right)^{++}\right)=Y .
$$

That is, intersecting the Zariski closure of $Y$ with $\left(\mathbb{R}^{d}\right)^{++}$gives no new points. In the singleton case, we have seen that this condition, real isolation, is sufficient. However, for general compact $Y$, it is no longer sufficient.

In fact, examples to illustrate this are ubiquitous (when $d>1$ ). The very simplest one I could think of is the following. Let $P=1+x y+x$ (the coefficients, here all ones, are not terribly important); then $\log P$ is the triangle with vertices $\{(0,0),(1,1),(1,0)\}$, and as rings $R_{P} \cong \mathbb{Z}[X, W]$ (the pure polynomial ring in two variables) via the transformation $X=x / P$ and $W=x y / P$. Let $f=(x-3)^{2}+(y-3)^{2}-1$, so $Z(f) \cap \mathbb{R}^{2}$ is the circle of radius one centred at $(3,3)$, and we set $Y$ to be this circle, sitting inside the positive quadrant of $\mathbb{R}^{2}$. In particular, $\log f=\{(0,0),(1,0),(2,0),(0,1),(0,2)\}$. It is trivial that $Z I(Y) \cap\left(\mathbb{R}^{2}\right)^{++}=Y$. However, there exists $\sigma \in \partial_{e} S\left(R_{P}, 1\right) \backslash Y$ such that $\sigma \mid \operatorname{ker} Y=0$.

Explicitly, $\sigma$ is the pure trace corresponding to the extreme point of cvx $\log P$ given by $(0,0) ; \sigma\left(g / P^{k}\right)=\left(g, x^{0,0}\right) /\left(P, x^{0,0}\right)^{k}$. Suppose $a=h / P^{k} \in R_{P}$; we may assume $\log h \subseteq \log P^{k}$. If $r \in Y$ implies $h(r) / P^{k}(r)=0$, that is, $\tau(a)=0$ for all $\tau \in Y$, then $h \mid Y \equiv 0$ (since $Y$ is in the positive quadrant, $P \mid Y$ vanishes nowhere). Hence there exists $e \in \mathbb{Q}[x, y]$ such that $h=e \cdot f\left(\right.$ as $\left.I_{\mathbb{Q}}(f)=f \mathbb{Q}[x, y]\right)$; multiplying by a positive integer $N$, we may assume $N h=e \cdot f$, where $e \in \mathbb{Z}[x, y]$.

We claim that this forces $h(0,0)=0$, that is, its constant term must be zero, from which it would follow that $\sigma(a)=0$, showing that $\operatorname{ker} Y \subset \operatorname{ker} \sigma$, as desired. If $h(0,0) \neq 0$, then as $\log h \subseteq \log P^{k}$, we would have to have $(0,0) \in \log h$, and in particular, this point is an extreme point of $\operatorname{cvx} \log h$. Since $(0,0)$ is also an extreme point of $\operatorname{cvx} \log f$, it easily follows that $(0,0)$ is an extreme point of $\operatorname{cvx} \log e$ (we are working with Laurent polynomials as opposed to ordinary polynomials, hence this complicated argument). Now consider the coefficients of $e$ and of $f$ restricted to the line $x=0$ (that is, throw away all the monomials with 
a power of $x), e_{0}$ and $f_{0}=y^{2}-6 y+17$. The product is not zero, and cannot be a single monomial (since $f_{0}$ is not), hence there must be, in addition to the constant term, a term of the form $y^{j}$ in the product. It is easy to check that this forces $(0, j) \in \log e \cdot f=\log h$. However, $\log P^{k}$ is contained in the lattice cone generated by $\{(0,0),(1,1),(1,0)\}$, which does not contain $(0, j)$. This contradicts $\log h \subseteq \log P^{k}$.

This example does not depend on the coefficients in $P$, that is, we could just as well have taken $P=2+3 x y+5 x$ (which guarantees that $R_{P}$ is approximately divisible), or whether we take $R_{P}$ or $R_{P} \otimes \mathbb{Q}$.

In contrast, if we take the same $f$, but $P=2+3 x+5 y$ (or with any other positive coefficients), then $f / P^{2} \in R_{P}$ and for all nonfaithful pure $\sigma$, we have $\sigma\left(f / P^{2}\right)>0$; hence the same $Y$ is now order unit good for $R_{P}$. This is part of a more general criterion.

Let $h$ be a polynomial in $d$ variables, and let $S$ be a finite set of lattice points in $\mathbb{Z}^{d}$, and $K(S)=\operatorname{cvx} S$. Suppose $F$ is a proper face of $K(S)$, and $\log h \subseteq k S$ (the set of sums of $k$ elements of $S$ ). We define $h_{F, k}$, the facial polynomial of $h$ relative to $F$ and $k$, by throwing away all the terms in $x^{w}$ of $h$ for which $w \notin k F$. In the case $S=\log P$, we can form the element $h_{F, k} /\left(P_{F}\right)^{k} \in R_{P_{F}}$ (in fewer variables, the number being the dimension of $F$ ). This yields a positive homomorphism $R_{P} \rightarrow R_{P_{F}}$ as described in [Handelman 1985].

Let $Y$ satisfy $Z I(Y) \cap\left(\mathbb{R}^{d}\right)^{++}=Y$, and form the ideal $I(Y)$ of $\mathbb{Z}\left[x_{1}, \ldots, x_{d}\right]$. Let $P$ be a projectively faithful polynomial in $\mathbb{Z}\left[x_{1}, \ldots, x_{d}\right]$. We say that $Y$ can be fitted with respect to $P$ if there exists a polynomial $h \in I(Y)$ such that

(a) $\log h \subseteq \log P^{k}$ for some $k$,

(b) for every proper face $F$ of $\mathrm{cvx} \log P, h_{F, k}$ has no negative coefficients.

This depends on $\log P$, but not so much on the coefficients $P$ [Handelman 1987, Proposition II.5].

Condition (b) can be somewhat weakened, since we are permitted to multiply the numerator and denominator of $h / P^{k}$ by powers of $P$, and apply eventual positivity criteria, e.g., [Handelman 1986]. The condition is equivalent to "for all pure $\sigma$ that are not faithful, there exists $h \in I(Y)$ such that $\sigma\left(h / P_{k}\right)>0$ ". For example, with $\log P=\{(0,0),(0,1),(1,0)\}$ and $Y$ the circle in $\left(\mathbb{R}^{2}\right)^{++}$of radius 1 centred at $(3,3), Y$ is fitted with respect to $P$. Just observe that $f$ has the three facial polynomials (corresponding to the three edges of $\operatorname{cvx} \log P$ (the extreme points take care of themselves, so we need not worry about the zero-dimensional faces), $(x-3)^{2}+17,(y-3)^{2}+17, x^{2}+y^{2}$. If we multiply the first two by a sufficiently high power, say $N$, of $1+x$ (respectively $(1+y)$ ), the outcome will have no negative coefficients. It follows that if $h=P^{N} f$, then $h$ will be positively fitted with respect to $P$, with $k=N+2$. 
Now the following is practically tautological.

Proposition 5.10. Let $P$ be a faithfully projective element of $\mathbb{Z}\left[x_{i}\right]$, and $Y$ a compact subset of $\left((\mathbb{R})^{d}\right)^{++}$. Then $Y$ generates an order unit good face for $R_{P}$ (and simultaneously for $\left.R_{P} \otimes \mathbb{Q}\right)$ if and only if

(i) $Z I(Y) \cap\left(\mathbb{R}^{d}\right)^{++}=Y$ and

(ii) $Y$ can be fitted with respect to $P$.

Conditions on $Y$ to guarantee property (b) of Lemma 5.9 seem to be very difficult, involving divisibility of polynomials (and so depend on the coefficients). So goodness of subsets of $\partial_{e} S\left(R_{P}, 1\right)$ is still problematic.

\section{Appendix A: Connections with zero-dimensional topological dynamics}

The referee has observed that this paper uses methods almost entirely from partially ordered abelian groups and Choquet theory, and its results refer to the former. As a result, the connections with dynamics are invisible. This informal appendix is intended to outline some of the connections. We assume that the reader has some knowledge of Cantor dynamical systems.

Let $(X, T)$ be a nonatomic zero-dimensional compact separable Hausdorff space (a Cantor set) together with a self-homeomorphism; we call this pair a Cantor minimal system. We may functorially attach a partially preordered abelian group, $K_{0}(X, T)$, to $(X, T)$, in any of several equivalent ways, e.g., the preordered Grothendieck group of the crossed-product $\mathrm{C}^{*}$-algebra $C(X) \times_{T} \mathbb{Z}$, or directly by computing the preordered Čech cohomology, $C(X, \mathbb{Z}) /(I-T) C(X, \mathbb{Z})$ (where $T$ has its natural action on $C(X, \mathbb{Z}))$, with the quotient preordering.

When $T$ is minimal (an abbreviation for the action of $T$ on $X$ is minimal), not only is the preordering a genuine partial ordering, but $K_{0}(X, T)$ is a simple dimension group; moreover (in the minimal case), together with a distinguished order unit, it is a complete invariant for strong orbit equivalence, and a complete invariant for orbit equivalence is obtained from the simple dimension group $K_{0}(X, T) / \operatorname{Inf}\left(K_{0}(X, T)\right)$ [Giordano et al. 1995; Herman et al. 1992].

When $T$ is no longer minimal, chain recurrence (a weak condition) will guarantee that $K_{0}(X, T)$ is partially ordered [Boyle and Handelman 1996]. Unfortunately, even for rather natural systems, such as shifts of finite type, $K_{0}(X, T)$ need not be a dimension group [Kim et al. 2001], and more recent work suggests that being a dimension group is a relatively rare phenomenon - moreover, not all (countable) dimension groups can appear as a $K_{0}(X, T)$. This calls into question the usefulness of the results here, since we work almost exclusively with dimension groups. 
Fortunately, many questions in nonminimal cases can be reduced to questions concerning dimension groups. This is because of the following result of Boyle and the author.

Theorem. If $T$ is chain recurrent, then there exists a dimension group $G$ together with an onto, order-preserving group homomorphism $\phi: G \rightarrow K_{0}(X, T)$ such that

(a) $\operatorname{ker} \phi \subseteq \operatorname{Inf} G$, and

(b) $\phi\left(G^{+}\right)=K_{0}(X, T)^{+}$.

Not only does this say that $K_{0}(X, T)$ is order-isomorphic to $G / \operatorname{ker} \phi$ with the quotient ordering on the latter, but it also implies that $\phi$ induces a natural affine homeomorphism between $S(G, u)$, the normalized trace space of $G$, and the normalized trace space of $K_{0}(X, T)$, which itself is just the space of invariant probability measures on $X$. The images of $G$ and of $K_{0}(X, T)$ in their affine representation agree, and this means that some properties of measures/traces transfer between $(X, T)$ and $G$ (a dimension group). For example, order unit goodness is the same whether we take $K_{0}(X, T)$ or $G$.

This allows one to transfer problems about traces (or finite invariant measures) on $K_{0}(X, T)$ to the dimension group $G$. As a simple example (already a known consequence of Krieger's marker lemma), if $T$ has no periodic points, then the image of $K_{0}(X, T)$ in its affine representation (taking as order unit $u=\left[\chi_{X}\right]$ ) is dense. In particular, almost divisibility transfers (near divisibility probably does not). More relevantly, order unit goodness and its refinable counterpart transfer completely between the two ordered groups, as does the purity criterion of [Goodearl and Handelman 1980]. Properties involving order ideals do not do so well, but very often there is a one-way implication. There is obviously more to be done.

Tensor products. Tensor products of dimension groups, or more generally, of partially ordered abelian groups, as discussed in Section 2, arise naturally. However, their translation to dynamical systems is not so clear. Nonetheless, there are examples - every minimal Cantor system can be realized as a continuous adic map on a Bratteli diagram, and the Cartesian product of the two Cantor sets admits an adic map compatible with the tensor product [BeH 2014, Appendix A].

There is a less tenuous interrelation. Let $(X, T)$ and $(Y, S)$ be Cantor dynamical systems (not necessarily minimal, although not much is known in the nonminimal case), which are at least chain recurrent. Form the product, $(X \times Y, T \times S)$ (meaning the $\mathbb{Z}$-action, not the $\mathbb{Z}^{2}$-action). There is a natural order-preserving group homomorphism, $\Phi: K_{0}(X \times Y, T \times S) \rightarrow K_{0}(X, T) \otimes K_{0}(Y, S)$ (with the usual positive cone on the tensor product). This is induced by the isomorphism $C(X \times Y, \mathbb{Z}) \cong C(X, \mathbb{Z}) \otimes C(Y, \mathbb{Z})$, the latter factoring onto $K_{0}(X, T) \otimes K_{0}(Y, S) ;$ 
then $(\mathrm{I}-(T \times S))(C(X \times Y, \mathbb{Z})) \subseteq(\mathrm{I}-T) C(X \times Y, \mathbb{Z})+(\mathrm{I}-S)(C(X \times Y, \mathbb{Z}))$ shows that $\Phi$ is well-defined.

Call the system constructed in [BeH 2014, Appendix A] realizing the tensor product, $(X \times Y, R)$. For the following observation, we don't really need the construction of $R$, merely that such a minimal system (realizing the tensor product of the dimension groups) exists, which is a consequence of [Giordano et al. 1995].

Observation. Suppose $(X, T)$ and $(Y, S)$ are minimal Cantor dynamical systems. In the following, any of (b), (c), or (d) implies that $T \times S$ is minimal. Moreover, (b), (c), and (d) are equivalent, and each implies (a); finally, if each of $(X, T)$ and $(Y, S)$ has only finitely many ergodic measures, then (a) implies (b).

(a) $T \times S$ is orbit equivalent to $R$.

(b) The kernel of the natural order-preserving homomorphism

$$
\Phi: K_{0}(X \times Y, T \times S) \rightarrow K_{0}(X, T) \otimes K_{0}(Y, S)
$$

consists of infinitesimals.

(c) Every invariant $(T \times S)$-ergodic measure is of the form $\mu \times v$, where $\mu$ is an invariant measure on $(X, T)$ and $v$ is an invariant measure on $(Y, S)$.

(d) For every continuous $f: X \rightarrow \mathbb{Z}$ of $X$ and every coboundary $h=(\mathrm{I}-S) g$ of $Y$ (where $g: Y \rightarrow \mathbb{Z}$ is continuous), $[f \cdot h]$ is an infinitesimal in $K_{0}(X \times Y, T \times S)$.

Remark. The apparent asymmetry in $X$ and $Y$ of (d) is illusory, as

$$
(\mathrm{I}-T \times S)(f \cdot g)=f \cdot h+((\mathrm{I}-T) f) \cdot(g \circ S) .
$$

Remark. Without the assumption that $(X, T)$ and $(Y, S)$ have only finitely many ergodic measures, it is still very likely true that (a) implies (b) anyway.

Proof. We show that (c) implies minimality of the product, and then that (b), (c), and (d) are equivalent in general. If $T \times S$ were not minimal, there would be a proper closed invariant subset $A \subset X \times Y$. Then any invariant ergodic measure supported on $A$ cannot be a product measure, and of course, one exists.

(b) implies (c): Since the kernel of the map to the tensor product is onto and has only infinitesimals in its kernel, it induces a homeomorphism on the normalized trace spaces, and clearly product traces map to product traces under this; since all pure traces on the tensor product are of the form $\sigma \otimes \tau$, where $\sigma$ and $\tau$ are pure traces on the two components respectively, it implies every pure trace on $K_{0}(X \times Y, T \times S)$ is a product trace, and this translates to product measure.

(c) implies (d): For any product measure on $X \times Y, d \rho=d \mu d \nu$, we have

$$
\int_{X \times Y} f \cdot h d \rho=\left(\int_{X} f d \mu\right) \cdot\left(\int_{Y} h d v\right)=0 .
$$


From (c), the closed convex hull of the product invariant measures is the set of all invariant measures, so $\int_{X \times Y} f \cdot h d \zeta=0$ for every invariant measure $\zeta$ on $X \times Y$. Hence $[f \cdot h]$ vanishes at every trace on $K_{0}(X \times Y, T \times S)$, and thus is an infinitesimal. (d) implies (b): The kernel of the map $K_{0}(X \times Y, T \times S) \rightarrow K_{0}(X, T) \otimes K_{0}(X, S)$ is spanned by the images (in $K_{0}$ ) of

$(\mathrm{I}-T \times S) C(X \times Y, \mathbb{Z}), \quad C(X, \mathbb{Z}) \cdot(\mathrm{I}-S)(C(Y, \mathbb{Z})), \quad(\mathrm{I}-T)(C(X, \mathbb{Z})) \cdot C(Y, \mathbb{Z})$.

By the remark, every element of $(\mathrm{I}-T)(C(X, \mathbb{Z})) \cdot C(Y, \mathbb{Z})$ belongs to the abelian group generated by the other two, whose images obviously land in the infinitesimal subgroup.

(b) implies (a): Factoring out the infinitesimals from both groups yields a unital order isomorphism (since the original map sends the positive elements onto the positive elements), so $T \times S$ is orbit equivalent to $R$.

(a) implies (b) (if each of $(X, T)$ and $(Y, T)$ has only finitely many ergodic measures): Let $m$ and $n$ be the respective number of pure traces (ergodic measures). The number of pure traces on the tensor product is exactly $m n$, and orbit equivalence implies the same number of pure traces for $K_{0}(X \times Y, T \times S)$. The natural map to the tensor product induces a positive map between the corresponding affine function spaces (of the same dimension, $m n$ ), which must therefore be an isomorphism. Hence every trace on $K_{0}(X \times Y, T \times S)$ factors through the tensor product. Thus the kernel of the map must be contained in the kernel of all the traces, and hence is contained in the infinitesimal subgroup.

Bernoulli measures and xerox actions. The results of Section 3 are reminiscent of those of [Akin et al. 2008], characterizing goodness of Bernoulli measures. Let $\left\{e_{i}\right\}$ be the standard basis for $\mathbb{Z}^{n}$, and set $x_{i}=x^{e_{i}}$ (in monomial notation), and $P=1+\sum_{i=1}^{n} x_{i}$ (for notational convenience, we sometimes write $1=x^{e_{0}}$, where $e_{0}=0$ ). Form $R_{P}$, a very special case of the ordered rings discussed in this section. This particular one is ring isomorphic to the pure polynomial ring, $A_{P}=\mathbb{Z}\left[X_{1}, \ldots, X_{n}\right]$, under the assignment $X_{i}=x_{i} / P$, and the positive cone is generated multiplicatively and additively by $\left\{X_{1}, \ldots, X_{n} ; 1-\sum X_{i}\right\}$.

The pure traces of $A_{P}$ are precisely the multiplicative ones, determined by $X_{i} \mapsto p_{i}$, where $0 \leq p_{i}$ and $\sum p_{i} \leq 1$. Let $p=\left(p_{1}, \ldots, p_{n}\right)$ denote the corresponding point in the standard simplex in $\mathbb{R}^{n}$. If $p_{0}: 1-\sum p_{i}>0$ (which occurs at least when the corresponding pure trace is faithful, and in other cases as well), we can reconstruct the point in $\left(\mathbb{R}^{n}\right)^{+}$(the faithful pure trace of $R_{P}$ whence it came; explicitly, $x_{i} \mapsto p_{i} / p_{0}$ ), and when the measure is faithful (meaning all of $p_{i}$ and $p_{0}$ are nonzero), these run over the entire open orthant, $\left(\mathbb{R}^{n}\right)^{++}$.

For all choices of (faithfully projective) $P$, there is a natural map from the pure trace space of $\left(R_{P}, 1\right)$ to the Newton polytope of $P$, given by the weighted moment 
map; because this particular choice for $P$ is so pleasant, the weighted moment map is particularly explicit. (For generic $P, R_{P}$ is not even a unique factorization domain - for example, if $P$ is irreducible over the integers, faithfully projective, and $R_{P}$ satisfies unique factorization, then up to the natural action of $\operatorname{AGL}(n, \mathbb{Z})$ on the exponents, $P=\sum_{i=0}^{n} a_{i} x_{i}$, where $x_{0}=1$ and $a_{i}$ are positive integers.)

Now back to our specific choice of $P$; this $R_{P}$ is not approximately divisible (for example, $X_{i} \mapsto 0$ yields a $\mathbb{Z}$-valued trace on $A_{P}$; we could fix this if we permitted nonmonic coefficients at all of the vertices). Nonetheless, we can analyze conditions (1) and (2) here.

For the point $\left.r:=\left(p_{i} / p_{0}\right) \in \mathbb{R}^{n}\right)^{++}, \tau_{r}$ will satisfy (1) and (2) precisely if $r$ is really isolated and $p_{0} / p_{i} \in \mathbb{Z}\left[p_{j} / p_{0}\right]=\tau_{r}\left(R_{P}\right)$; in particular, $p_{0} / p_{i}$ are units in $\tau_{r}\left(R_{P}\right)$ and are algebraic. Since $\sum_{1}^{n} p_{i}=1-p_{0}$, we quickly deduce that all of $p_{0}, p_{1}, \ldots, p_{n}$ must be algebraic and are units in $\mathbb{Z}\left[p_{0}, p_{1}, \ldots, p_{n}\right]$ (the image of $A_{P}$ under the corresponding trace).

The density matrices that implement the pure traces on the fixed point $\mathrm{C}^{*}$-algebras are exactly those whose diagonals are $p$. In a sense, $\mathrm{C}^{*}$-algebra traces are the noncommutative analogue of Bernoulli measures.

Strict direct sums. There is no obvious connection between strict direct sums (even for simple dimension groups) and dynamical systems. In fact, although we know that a strict direct sum of dimension groups is again a dimension group, given realizations of the two components (as direct limits, that is, given Bratteli diagrams for each of them), there is no way known to construct the strict direct sum as such a direct limit from the two direct limits (that is, there does not seem to be a way of finding a Bratteli diagram based on the two given ones). There are a few (very few) ad hoc constructions in very special cases.

\section{Appendix B: Order unit good traces on $\mathbb{Z}^{k}$}

The criteria for goodness of traces on nearly divisible dimension groups depend on order unit goodness; and the usefulness of the former is a consequence of the relatively simple characterization of order unit good traces on approximately divisible dimension groups, namely density of the image of $\operatorname{ker} \tau$ in $\tau^{\vdash}$ via the affine representation of $(G, u)$.

To obtain useful criteria for goodness on a larger class of dimension groups, it would be helpful to find an analogous characterization of order unit goodness in the presence of discrete traces. In this appendix, we consider the extreme dimension groups with discrete traces, namely the simplicial ones, $\mathbb{Z}^{k}$, with the usual ordering. It is already known that up to scalar multiple, the only good traces are given by left multiplication by a vector whose entries consist only of zeros and ones [Handelman 2013b, Lemma 6.2]. 
With the current definition of order unit good (really intended for approximately divisible groups), the order unit good traces on $\mathbb{Z}^{k}$ can be characterized, but the characterization makes it difficult to see how to obtain goodness criteria for more general dimension groups, as we did in the nearly divisible case.

Let $v \in\left(\mathbb{R}^{k \times 1}\right)^{+} \backslash\{\boldsymbol{0}\}$; then $v$ induces a trace on $\mathbb{Z}^{k}$, via left multiplication, $\phi_{v}: \mathbb{Z}^{k} \rightarrow \mathbb{R}$ sending $w \mapsto v w$ (we think of $\mathbb{Z}^{k}$ as a set of columns, so matrix multiplication makes sense). Obviously we can replace $v$ by any positive real multiple of itself without changing properties such as goodness or order unit goodness. In addition, we may apply any permutation to the entries, with the same lack of bad consequences. We may also discard any zeros (reducing the size of the vectors, that is, decreasing $k$ ).

Suppose $v$ has only integer entries; then we may order the nonzero entries, so that

$$
v=(n(1), n(2), \ldots, n(r) ; 0,0, \ldots, 0), \quad \text { where } n(1) \leq n(2) \leq \cdots .
$$

We may also assume that $\operatorname{gcd}\{n(i)\}=1$.

Lemma B.1. With this choice of $v$, we have that $\phi_{v}$ is order unit good if and only if $n(1)=1$ and for all $r \geq j>1$, we have $n(j) \leq 1+\sum_{i<j} n(i)$.

Proof. Assume $v$ is in the form indicated, and $\phi_{v}$ is order unit good. Since $\operatorname{gcd}\{n(i)\}=1$, there exists a vector $w$ such that $v w=1$. Set $u=(1,1,1, \ldots, 1)$; we have that $u$ is an order unit, hence it is $\phi_{v}$-order unit good. Since $v u>1$ (unless $v=(1,0,0, \ldots, 0)$ which is trivially good $)$, there must exist $w_{0} \in\left(\mathbb{Z}^{d}\right)^{+}$such that $v w_{0}=v w=1<v u$. Since the nonzero entries of $v$ are increasing, this forces the smallest one, $n(1)$, to be 1 . Hence $n(1)=1$.

Since $v u=\sum n(i):=N$, and there exists $w \in \mathbb{Z}^{k}$ such that $v w=1$, for each $s$ with $1<s<N$, there exists $w_{s} \in\{0,1\}^{k}$ (as $0 \leq w_{0} \leq u$ ) such that $v w_{s}=s$, by order unit goodness of $u$. Now suppose that for some $j$, we have $n(j)>1+\sum_{i<j} n(i)$. Then $n(j)-1$ cannot be realized as a sum of $n(i)$ s (using at most one for each choice of $i$ ), since $n(j)-1>\sum_{i<j} n(i)$, and $n(j) \leq n\left(j^{\prime}\right)$ for all $j^{\prime}>j$ (if there are any such $j^{\prime}$ ). Hence no such $w_{0}$ can exist.

Thus, if $u$ is $\phi_{v}$-order unit good, then the constraint on growth must hold.

Conversely, suppose the inequalities hold. It is then an easy induction argument (on $r$, augmenting the vector by adjoining $n(k+1)$ ) to show that $u$ is $\tau_{v}$-order unit good, by realizing every integer in the interval $(0, N)$. Finally, to show that every order unit is $\phi_{v}$-order unit good ( $u$ was the smallest choice), it suffices to show that if we add a single one to a $\phi_{v}$-order unit good vector, the outcome is again $\phi_{v}$-order unit good.

In particular, the choices for $v,(1,2,4,8,16)$ and $(1,1,1,4)$ yield order unit good traces, but $(1,3)$ and $(1,1,1,5)$ do not. This rather complicated set of conditions, when applied to order ideals in dimension groups that have a simplicial 
quotient by an order ideal, likely makes order unit goodness unusable for the purposes we had in mind.

Lemma B.2. If $\phi_{v}: \mathbb{Z}^{k} \rightarrow \mathbb{R}$ is an order unit good trace, then up to scalar multiple, $v \in\left(\mathbb{Z}^{k \times 1}\right)^{+}$.

Proof. In $\mathbb{Z}^{k}$, all intervals of the form $[0, u]$ (where $u$ is an order unit) are finite sets. If there were an irrational ratio among the nonzero entries of $v$, we would obtain $\phi_{v}\left(\mathbb{Z}^{k}\right) \cap[0, N]$ is infinite for any positive integer $N$. If order unit goodness held, this would be impossible. Hence all the ratios are rational, and it easily follows that after suitable scalar multiplication, we can convert $v$ to an integer row.

Proposition B.3. Let $v$ be an element of $\left(\mathbb{R}^{k}\right)^{+} \backslash\{\boldsymbol{\Gamma}\}$. Then $\phi_{v}$ is an order unit good trace if and only if up to scalar multiple and after rearrangement so that $v=(n(1), \ldots, n(r) ; 0,0, \ldots)$ with $n(i-1) \leq n(i)$, we have $n(i) \in \mathbb{N}, n(1)=1$, and for all $1<j \leq r$,

$$
n(j) \leq 1+\sum_{i<j} n(i)
$$

\section{Appendix C: Good simplices}

In the finite-dimensional case, we verify a conjecture from [BeH 2014, Section 7] that good subsets of Choquet simplices are obtained as coproducts of faces with singleton subsets of disjoint faces.

Let $K$ be a Choquet simplex. A nonempty subset $J$ of $K$ is said to be good (following [BeH 2014]) if it satisfies the following (redundant set of) properties:

(i) $J$ is a (compact) Choquet simplex.

(ii) There exists a closed flat $\mathcal{L}$ such that $J=\mathcal{L} \cap K$.

(iii) If $a \in \operatorname{Aff}(J)^{++}$and $b \in \operatorname{Aff}(K)^{++}$are such that $a \ll b \mid J$, then there exists $a^{\prime} \in \operatorname{Aff}(K)^{++}$such that $a^{\prime} \mid J=a$ and $a^{\prime} \ll b$.

We denote this relationship between $J$ and $K$ by $J{ }_{G}^{G} K$ (there is a $G$ inside the inclusion sign). If $F$ is a closed face of $K$, we denote it $F \triangleleft K$. A question arising out of [BeH 2014] is to characterize good subsets of Choquet simplices. For example, closed faces are good, and singleton sets are also good, and coproducts (within the category of simplices and good subsets) preserve these properties. A conjecture was made concerning the structure of good subsets; we verify this in the case that $K$ is finite-dimensional.

Now (ii) is redundant, and only the compact convex part of (i) is necessary. This is based on the following simple construction.

If $X$ is a subset of a real vector space, define the affine span of $X$, denoted Aspan $X$, as the set of finite sums $\left\{\sum r_{i} x_{i} \mid r_{i} \in \mathbb{R}, \sum r_{i}=1, x_{i} \in X\right\}$. 
If $J$ is a singleton or a line segment, there is (almost) nothing to do. Define $\mathcal{L}_{0}=\operatorname{Aspan} J$. If there exists $v \in\left(K \cap \mathcal{L}_{0}\right) \backslash J$, we can write $v=\sum \alpha_{i} v_{i}-\beta_{j} w_{j}$, where $v_{i}, w_{j} \in J$, and $\alpha_{i}, \beta_{j}>0$, and $\sum \alpha_{i}-\sum \beta_{j}=1$. We can also arrange that $\operatorname{cvx}\left\{v_{i}\right\} \cap \operatorname{cvx}\left\{w_{j}\right\}=\varnothing$. Hence for any positive $\eta<1$, there exists $a \in(\text { Aff } J)^{++}$ such that $1-\eta<a \mid \operatorname{cvx}\left\{w_{j}\right\}<1$ and $a \mid \operatorname{cvx}\left\{a_{i}\right\}<\eta$. Since $a$ is continuous, it is bounded above, so (iii) applies with some constant $b \in \operatorname{Aff} K$.

Hence there exists $a^{\prime} \in(\text { Aff } K)^{++}$such that $a=a^{\prime} \mid J$. Evaluating the equation at $a^{\prime}$, we obtain

$$
0<a^{\prime}(w)=\sum \alpha_{i} a\left(v_{i}\right)-\sum \beta_{j} a\left(w_{j}\right)<\eta \sum \alpha_{i}-(1-\eta) \sum \beta_{j} .
$$

This entails $\eta\left(\sum \alpha_{i}+\sum \beta_{j}\right)>\sum \beta_{j}$. Now $\sum \beta_{j}>0$, since otherwise $v \in J$. Hence we can choose at the outset positive $\eta<\sum \beta_{j} /\left(\sum \alpha_{i}+\sum \beta_{j}\right)$, which yields a contradiction.

Thus $\mathcal{L}_{0} \cap K=J$. If $x_{n} \in \mathcal{L}_{0}$ and $x_{n} \rightarrow x \in K$, but $x \notin J$, there exists a line segment joining $x$ to an element of the relative interior of $J$; it must pass through at least two points in $J$, hence $x \in \mathcal{L}_{0}$. In other words, with $\mathcal{L}$ equalling the closure of $\mathcal{L}_{0}$, we have $J=\mathcal{L}_{0} \cap K=\mathcal{L} \cap K$.

To check that the compact convex set $J$ must be a simplex if (iii) is satisfied, observe that the quotient Aff $K / J^{\vdash}$ (with the strict ordering on Aff $K$, $J^{\vdash}=\{a \in \operatorname{Aff} K|a| J \equiv 0\}$, and the quotient ordering) is order isomorphic to Aff $J$ (with the strict ordering). But goodness implies [BeH 2014] that it satisfies Riesz interpolation, which of course forces $J$ to be a Choquet simplex.

Let $K^{\prime}$ and $K^{\prime \prime}$ be simplices (simplices mean Choquet simplices; but most of the time we will working in finite dimensions, so simplex means the usual simplex) sitting inside some common simplex $K$ which in turn is contained in some topological vector space. Suppose that Aspan $K^{\prime} \cap$ Aspan $K^{\prime \prime}=\varnothing$; we write this as $K^{\prime} \wedge K^{\prime \prime}=\varnothing$. Then the closure of $\operatorname{cvx}\left(K^{\prime}, K^{\prime \prime}\right)$ is itself a simplex, and we refer to this as the coproduct, written $K^{\prime} \dot{\vee} K^{\prime \prime}$ (this is more an internal coproduct, but we shall not distinguish internal from external). If $K^{\prime}$ and $K^{\prime \prime}$ are faces of $K$, sufficient for $K^{\prime} \wedge K^{\prime \prime}=\varnothing$ is that their intersection be empty (since $K$ is a simplex); in this case, we say that $K^{\prime}$ and $K^{\prime \prime}$ are disjoint. If $\left\{K^{i}\right\}$ is a finite family of subsimplices, then disjointness of the set is defined inductively in the obvious way, so that $\dot{\bigvee}_{i} K^{i}$ makes sense and is a simplex.

We record elementary properties related to goodness.

Lemma C.1. (a) Suppose $J \mathbb{G} K$ and $K \mathbb{G} L$; then $J \mathbb{G} L$.

(b) If $F \triangleleft K$, then $F$ G $K$.

(c) If $J \mathbb{G} K$ and $F \triangleleft K$, then $J \cap F \triangleleft J$ and $J \cap F$ G $K$ whenever $J \cap F \neq \varnothing$.

(d) If $J_{i}$ G $K_{i}$ for $i=1,2$ and $K_{1} \wedge K_{2}=\varnothing$, then $J_{1} \dot{\vee} J_{2}$ (G $K_{1} \dot{\vee} K_{2}$. 
The crucial result is the following. Its proof rests heavily on finite-dimensionality, but is a minor modification of the previous argument.

Lemma C.2. Let $K$ be a finite dimensional simplex, and suppose $J$ (G $K$. Let $J_{1}$ and $J_{2}$ be disjoint faces of $J$. Set $F_{i}(i=1,2)$ to be the smallest face of $K$ that contains $J_{i}$. Then $F_{1}$ and $F_{2}$ are disjoint.

Proof. It suffices to show that $F_{1} \cap F_{2}=\varnothing$. If not, the intersection is a face, and hence contains a vertex (that is, extreme point) of $K$; call it $v$. We may suppose that $v \notin J_{2}$ (since $J_{1} \cap J_{2}=\varnothing$ ). Since $J$ is itself a finite-dimensional simplex and $J_{i}$ are disjoint faces, for any $\eta>0$ (which we will specify later), we may find $a \in \operatorname{Aff}(J)^{++}$such that $a\left|J_{2} \ll 1-\epsilon, a\right| J_{1} \ll \eta$, and $a \ll 1$ (on all of $J$ ). Set $b$ to be the constant function 1 on all of $K$, so that $0 \ll a \ll b \mid J$.

By goodness, there exists $a^{\prime} \in \operatorname{Aff}(K)^{++}$such that $a^{\prime} \ll b$ and $a^{\prime} \mid J=a$. It is now easy to show that for suitably small $\eta$ (depending on the boundary measure of elements of $J_{i} \subset F_{i}$ ), this leads to a contradiction.

Since $v \notin J$ and $F_{2}$ is the smallest face containing $J_{2}$, there must exist $w \in J_{2}$ such that $w=\lambda v+\sum_{s} \lambda_{s} v_{s}$, where $v_{s} \in \partial_{e} F_{2}, \lambda>0, \lambda_{s} \geq 0$ and $\lambda=1-\sum \lambda_{s}$. Evaluating at $a^{\prime}$, we obtain

$$
\lambda a^{\prime}(v)=a(w)-\sum \lambda_{s} a^{\prime}\left(v_{s}\right) \geq 1-\eta-(1-\lambda)
$$

(since $\left.a^{\prime}\left(v_{s}\right) \leq b\left(v_{s}\right)=1\right)$. Thus $a^{\prime}(v) \geq 1-\eta / \lambda$.

Now working within $F_{1}$, again since $F_{1}$ is the smallest face containing $J_{1}$, there must exist $y \in J_{1}$ such that $y=\mu v+\sum_{t} \mu_{t} y_{t}$, where $\left\{v, y_{t}\right\} \subseteq \partial_{e} F_{1}, \mu>0, \mu_{s} \geq 0$, and $\mu=1-\sum \mu_{s}$. Applying $a^{\prime}$, we obtain $\mu a^{\prime}(v)=a(y)-\sum \mu_{t} a^{\prime}\left(y_{t}\right)<\eta$. Hence $a^{\prime}(v)<\eta / \mu$.

Thus the two inequalities force $\eta / \mu+\eta / \lambda>1$. We reach a contradiction if we choose $\eta<1 /(1 / \mu+1 / \lambda)$.

One obstruction (among several) to extending this to infinite-dimensional simplices is the fact that the representing measures of relative interior points might vanish on the intersection of the faces. We would also have to restrict to closed faces in this case (since otherwise it is not clear that the smallest face exists), and this presents problems.

Let $\left\{F_{i}\right\}$ be a disjoint collection of faces - that is, for all $i, F_{i} \wedge\left(\dot{\bigvee}_{j \neq i} F_{j}\right)=\varnothing-$ of the simplex $K$, and for each $i$, let $v_{i}$ be a point in the relative interior of $F_{i}$; we also assume that the $F_{i}$ are not themselves singletons. We may form $J_{0}:=\operatorname{cvx}\left\{v_{i}\right\}$ and $F_{0}:=\operatorname{cvx}\left\{F_{i}\right\}$; of course, this is the coproduct of $\left(\left\{v_{i}\right\}, F_{i}\right)$, and $J_{0}$ is thus a good subset of $F_{0}$ (since each $\left.\left\{v_{i}\right\} \in \mathbb{G} F_{i}\right)$. As in [BeH 2014], we call the $\left(v_{i}, F_{i}\right)$, together with $(F, F)$ (that is, the face $F \mathbb{G} F)$ building blocks. It was conjectured (in the finite-dimensional case) that if $J \mathbb{G} K$, then there exists a face $F$ of $K$, together with a disjoint face $F_{0}$ obtained as the coproduct, such that $J=F \dot{\vee} J_{0}$; in other 
words, that coproducts of the building blocks yield all good subsets; alternatively, that there is a maximal face $F$ of $K$ sitting inside $J$, and $J$ is obtained by taking coproducts with respective singleton sets sitting inside pairwise disjoint faces. This now follows easily.

Corollary C.3. Suppose $K$ is a finite-dimensional simplex and $J \mathbb{G} K$. Then there exist a (possibly empty) face $F$ of $K$ together with a finite set of faces $F_{i}$ of dimension at least one such that $\left\{F, F_{1}, \ldots\right\}$ is disjoint, together with $v_{i}$ in the relative interior of $F_{i}$ such that $J=\operatorname{cvx}\left\{F, v_{i}\right\}$.

Proof. We proceed by induction on the dimension of $J$. Let $F$ be the convex hull of all the vertices of $K$ that lie in $J$; these are automatically vertices of $J$. If this exhausts the vertices of $J$, then $F=J$ and $F$ is a face (since $K$ is a finite-dimensional simplex), and there is nothing to do. Of course, $F$ can be empty.

Otherwise, there exists a vertex $v_{1}$ of $J$ that is not in $\partial_{e} K$; necessarily this belongs to a proper face (it cannot be in the interior, in fact by property (ii), but this can also be proved using only (i) and (iii)) of $K$, and let $F_{1}$ be the smallest face of $K$ containing $v_{1}$. Then $v_{1}$ is in the relative interior of $F_{1}$. Let $J^{1}$ be the complementary face to $\left\{v_{1}\right\}$ in $J$ (that is, the convex hull of all the other vertices of $J$ ).

If $J^{1}$ is empty, then $J=J^{1}$ is already a singleton, and we are done.

If $J^{1}$ is not empty, then $J^{1} \triangleleft J$, so $J^{1} \mathbb{G}_{\mathbb{G}} J$, and thus by transitivity, $J_{1} \mathbb{G} K$. We can apply the previous lemma. Let $F^{1}$ be the smallest face of $K$ containing $J^{1}$; then $F^{1} \cap F_{1}=\varnothing$, and thus $J$ decomposes as the coproduct of $J^{1}$ and $\left\{v_{1}\right\}$ (using faces $F^{1}$ and $F_{1}$ ), so by induction on the dimension of $J$, and we are done.

\section{Acknowledgment}

Discussions with my colleague Damien Roy concerning the material in Section 3 were very helpful.

\section{References}

[Akin 1999] E. Akin, "Measures on Cantor space", Topology Proc. 24:Summer (1999), 1-34. MR 2002j:28002 Zbl 1025.28007

[Akin 2005] E. Akin, "Good measures on Cantor space”, Trans. Amer. Math. Soc. 357:7 (2005), 2681-2722. MR 2006e:37003 Zbl 1078.37004

[Akin et al. 2008] E. Akin, R. Dougherty, R. D. Mauldin, and A. Yingst, "Which Bernoulli measures are good measures?”, Colloq. Math. 110:2 (2008), 243-291. MR 2009f:37009 Zbl 1151.37011

[Bezuglyi and Handelman 2014] S. Bezuglyi and D. E. Handelman, "Measures on Cantor sets: the good, the ugly, the bad", Trans. Amer. Math. Soc. 366:12 (2014), 6247-6311. MR 3267010 Zbl 06371207

[Boyle and Handelman 1996] M. Boyle and D. E. Handelman, "Orbit equivalence, flow equivalence and ordered cohomology”, Israel J. Math. 95 (1996), 169-210. MR 98a:46082 Zbl 0871.58071 
[Effros et al. 1980] E. G. Effros, D. E. Handelman, and C.-L. Shen, "Dimension groups and their affine representations", Amer. J. Math. 102:2 (1980), 385-407. MR 83g:46061 Zbl 0457.46047

[Frick and Ormes 2013] S. B. Frick and N. Ormes, "Dimension groups for polynomial odometers", Acta Appl. Math. 126 (2013), 165-186. MR 3077947 Zbl 06210545

[Giordano et al. 1995] T. Giordano, I. F. Putnam, and C. F. Skau, "Topological orbit equivalence and $C^{*}$-crossed products”, J. Reine Angew. Math. 469 (1995), 51-111. MR 97g:46085 Zbl 0834.46053

[Giordano et al. $\geq 2016]$ T. Giordano, D. E. Handelman, and M. Hosseini, "Orbit equivalence of Cantor minimal systems and their continuous spectra", In preparation.

[Goodearl 1986] K. R. Goodearl, Partially ordered abelian groups with interpolation, Mathematical Surveys and Monographs 20, American Mathematical Society, Providence, RI, 1986. MR 88f:06013 Zbl 0589.06008

[Goodearl and Handelman 1980] K. R. Goodearl and D. E. Handelman, "Metric completions of partially ordered abelian groups”, Indiana Univ. Math. J. 29:6 (1980), 861-895. MR 82b:06020 Zbl 0455.06012

[Goodearl and Handelman 1986] K. R. Goodearl and D. E. Handelman, "Tensor products of dimension groups and $K_{0}$ of unit-regular rings", Canad. J. Math. 38:3 (1986), 633-658. MR 87i:16043 Zbl 0608.16027

[Handelman 1982] D. E. Handelman, "Free rank $n+1$ dense subgroups of $\mathbb{R}^{n}$ and their endomorphisms”, J. Funct. Anal. 46:1 (1982), 1-27. MR 84h:06019 Zbl 0489.20044

[Handelman 1985] D. E. Handelman, Positive polynomials and product type actions of compact groups, Mem. Amer. Math. Soc. 54:320, American Mathematical Society, Providence, RI, 1985. MR 86h:46091 Zbl 0571.46045

[Handelman 1986] D. E. Handelman, "Deciding eventual positivity of polynomials", Ergodic Theory Dynam. Systems 6:1 (1986), 57-79. MR 87m:26013 Zbl 0606.22005

[Handelman 1987] D. E. Handelman, Positive polynomials, convex integral polytopes, and a random walk problem, Lecture Notes in Mathematics 1282, Springer, Berlin, 1987. MR 89b:06014 Zbl 0654.46059

[Handelman 2009] D. E. Handelman, "Matrices of positive polynomials", Electron. J. Linear Algebra 19 (2009), 2-89. MR 2011g:46105 Zbl 1195.37010

[Handelman 2013a] D. E. Handelman, "Invariants for critical dimension groups and permutationHermite equivalence”, preprint, 2013. arXiv 1309.7417

[Handelman 2013b] D. E. Handelman, "Realizing dimension groups, good measures, and Toeplitz factors”, Illinois J. Math. 57:4 (2013), 1057-1109. MR 3285868 Zbl 1311.46060

[Handelman 2014] D. E. Handelman, "Limits of simple dimension groups and weakly initial objects", Indiana Univ. Math. J. 63:5 (2014), 1567-1600. MR 3283562 Zbl 06388780

[Herman et al. 1992] R. H. Herman, I. F. Putnam, and C. F. Skau, "Ordered Bratteli diagrams, dimension groups and topological dynamics”, Internat. J. Math. 3:6 (1992), 827-864. MR 94f:46096 Zbl 0786.46053

[Kim et al. 2001] K. H. Kim, F. W. Roush, and S. G. Williams, "Duality and its consequences for ordered cohomology of finite type subshifts", pp. 243-265 in Combinatorial \& computational mathematics (Pohang, 2000), edited by S. Hong et al., World Scientific, River Edge, NJ, 2001. MR 2003a:37016 Zbl 1002.37006

[Medynets 2006] K. Medynets, "Cantor aperiodic systems and Bratteli diagrams", C. R. Math. Acad. Sci. Paris 342:1 (2006), 43-46. MR 2006g:37011 Zbl 1081.37004 
[Petersen 2012] K. Petersen, "An adic dynamical system related to the Delannoy numbers", Ergodic Theory Dynam. Systems 32:2 (2012), 809-823. MR 2901372 Zbl 1257.37006

[Putnam 1989] I. F. Putnam, "The $C^{*}$-algebras associated with minimal homeomorphisms of the Cantor set”, Pacific J. Math. 136:2 (1989), 329-353. MR 90a:46184 Zbl 0631.46068

[Renault 1980] J. Renault, A groupoid approach to $C^{*}$-algebras, Lecture Notes in Mathematics 793, Springer, Berlin, 1980. MR 82h:46075 Zbl 0433.46049

Received September 4, 2014. Revised August 2, 2015.

DAVID HANDELMAN

MATHEMATICS DEPARTMENT

UNIVERSITY OF OTTAWA

585 KING EDWARD

OTTAWA K1N 6N5 ON

CANADA

dehsg@uottawa.ca 


\title{
PACIFIC JOURNAL OF MATHEMATICS
}

\author{
msp.org/pjm
}

Founded in 1951 by E. F. Beckenbach (1906-1982) and F. Wolf (1904-1989)

\section{EDITORS}

Don Blasius (Managing Editor)

Department of Mathematics

University of California

Los Angeles, CA 90095-1555

blasius@math.ucla.edu

\author{
Paul Balmer \\ Department of Mathematics \\ University of California \\ Los Angeles, CA 90095-1555 \\ balmer@math.ucla.edu \\ Robert Finn \\ Department of Mathematics \\ Stanford University \\ Stanford, CA 94305-2125 \\ finn@math.stanford.edu \\ Sorin Popa \\ Department of Mathematics \\ University of California \\ Los Angeles, CA 90095-1555 \\ popa@math.ucla.edu
}

\author{
Vyjayanthi Chari \\ Department of Mathematics \\ University of California \\ Riverside, CA 92521-0135 \\ chari@math.ucr.edu \\ Kefeng Liu \\ Department of Mathematics \\ University of California \\ Los Angeles, CA 90095-1555 \\ liu@math.ucla.edu \\ Jie Qing \\ Department of Mathematics \\ University of California \\ Santa Cruz, CA 95064 \\ qing@ cats.ucsc.edu
}

\section{PRODUCTION}

Silvio Levy, Scientific Editor, production@msp.org

\section{SUPPORTING INSTITUTIONS}

ACADEMIA SINICA, TAIPEI

CALIFORNIA INST. OF TECHNOLOGY

INST. DE MATEMÁTICA PURA E APLICADA

KEIO UNIVERSITY

MATH. SCIENCES RESEARCH INSTITUTE

NEW MEXICO STATE UNIV.

OREGON STATE UNIV.

\author{
STANFORD UNIVERSITY \\ UNIV. OF BRITISH COLUMBIA \\ UNIV. OF CALIFORNIA, BERKELEY \\ UNIV. OF CALIFORNIA, DAVIS \\ UNIV. OF CALIFORNIA, LOS ANGELES \\ UNIV. OF CALIFORNIA, RIVERSIDE \\ UNIV. OF CALIFORNIA, SAN DIEGO \\ UNIV. OF CALIF., SANTA BARBARA
}

\author{
Daryl Cooper \\ Department of Mathematics \\ University of California \\ Santa Barbara, CA 93106-3080 \\ cooper@math.ucsb.edu \\ Jiang-Hua Lu \\ Department of Mathematics \\ The University of Hong Kong \\ Pokfulam Rd., Hong Kong \\ jhlu@maths.hku.hk \\ Paul Yang \\ Department of Mathematics \\ Princeton University \\ Princeton NJ 08544-1000 \\ yang@math.princeton.edu
}

These supporting institutions contribute to the cost of publication of this Journal, but they are not owners or publishers and have no responsibility for its contents or policies.

See inside back cover or msp.org/pjm for submission instructions.

The subscription price for 2016 is US $\$ 440 /$ year for the electronic version, and $\$ 600 /$ year for print and electronic.

Subscriptions, requests for back issues and changes of subscribers address should be sent to Pacific Journal of Mathematics, P.O. Box 4163, Berkeley, CA 94704-0163, U.S.A. The Pacific Journal of Mathematics is indexed by Mathematical Reviews, Zentralblatt MATH, PASCAL CNRS Index, Referativnyi Zhurnal, Current Mathematical Publications and Web of Knowledge (Science Citation Index).

The Pacific Journal of Mathematics (ISSN 0030-8730) at the University of California, c/o Department of Mathematics, 798 Evans Hall \#3840, Berkeley, CA 94720-3840, is published twelve times a year. Periodical rate postage paid at Berkeley, CA 94704, and additional mailing offices. POSTMASTER: send address changes to Pacific Journal of Mathematics, P.O. Box 4163, Berkeley, CA 94704-0163.

PJM peer review and production are managed by EditFLOW ${ }^{\circledR}$ from Mathematical Sciences Publishers.

\section{PUBLISHED BY}

\section{mathematical sciences publishers \\ nonprofit scientific publishing}

http://msp.org/

(C) 2016 Mathematical Sciences Publishers 


\title{
PACIFIC JOURNAL OF MATHEMATICS
}

\author{
Volume $281 \quad$ No. $2 \quad$ April 2016
}

The Eisenstein elements of modular symbols for level product of two 257 distinct odd primes

DEBARGHA BANERJEE and SRILAKSHMI KRISHNAMOORTHY

Primitively generated Hall algebras

ARKADY BERENSTEIN and JACOB GREENSTEIN

Generalized splines on arbitrary graphs

Simcha Gilbert, JULiAnNA TyMOCZKO and SHIRA ViEL

Good traces for not necessarily simple dimension groups

DAVID HANDELMAN

On Fourier coefficients of certain residual representations of symplectic groups

DIHUA JIANG and BAIYING LIU

On the existence of central fans of capillary surfaces

AMMAR KHANFER

Surfaces of prescribed mean curvature $H(x, y, z)$ with one-to-one central projection onto a plane

FRIEDRICH SAUVIGNY 